\title{
Valence Bond Ground States in Isotropic Quantum Antiferromagnets
}

\author{
Ian Affleck ${ }^{1, \star}$, Tom Kennedy ${ }^{2, \star \star}$, Elliott H. Lieb ${ }^{2, \star \star \star}$, and Hal Tasaki ${ }^{2, \star \star \star}$ \\ ${ }^{1}$ Department of Physics, University of British Columbia, Vancouver, B.C., V6T2A6, Canada \\ 2 Department of Physics, Princeton University, P.O. Box 708, Princeton, NJ 08544, USA
}

\begin{abstract}
Haldane predicted that the isotropic quantum Heisenberg spin chain is in a "massive" phase if the spin is integral. The first rigorous example of an isotropic model in such a phase is presented. The Hamiltonian has an exact $S O$ (3) symmetry and is translationally invariant, but we prove the model has a unique ground state, a gap in the spectrum of the Hamiltonian immediately above the ground state and exponential decay of the correlation functions in the ground state. Models in two and higher dimension which are expected to have the same properties are also presented. For these models we construct an exact ground state, and for some of them we prove that the two-point function decays exponentially in this ground state. In all these models exact ground states are constructed by using valence bonds.
\end{abstract}

\section{Table of Contents}

1. Introduction . . . . . . . . . . . . . . . . . 478

2. The One-Dimensional Model (1.1)

2.1. The Ground State . . . . . . . . . . . . . . . . . . . . . . . . . 481

2.2. The Ground State Two-Point Correlation Function . . . . . . . . . . . 485

2.3. The Energy Gap. . . . . . . . . . . . . . . . . . . . . . . . . 487

2.4. The Infinite Chain . . . . . . . . . . . . . . . . . . . . . . . . . . . . 492

3. The Spin 3/2 Model on the Hexagonal Lattice

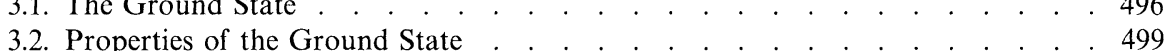

3.3. Proof of Exponential Decay

4. VBS States on an Arbitrary Lattice

4.1. The Ground States . . . . . . . . . . . . . . . . . . . . . . . . . 504

4.2. The VBS State on the Cayley Tree . . . . . . . . . . . . . . . . . 506

4.3. $S U(n)$ VBS State and Random Loop Representation . . . . . . . . . . . 510

4.4. $S U(n)$ Quantum "Spin" System . . . . . . . . . . . . . . . . . . . . . 514

* Supported in part by N.S.F. Grant PHY-80-19754. Fellow of the A.P. Sloan Foundation and the Canadian Institute for Advanced Research

$\star \star$ N.S.F. Post-doctoral Fellow

$\star \star \star$ Supported in part by N.S.F. Grant PHY-85-15288-A01 
5. Energy Gap in the Majumdar-Ghosh Model

5.1. The Model and the Ground States . . . . . . . . . . . . . . . . . 516

5.2. The Energy Gap. . . . . . . . . . . . . . . . . . . . . . . . 518

5.3. The Infinite Chain . . . . . . . . . . . . . . . . . . . . . . 520

6. Other Valence Bond Ground States . . . . . . . . . . . . . . . . . . 522

Appendix A. Néel Order in the Hexagonal Lattice Heisenberg Model . . . . . . . 524

\section{Introduction}

The ground state properties of quantum antiferromagnets not only have important physical consequences, they also contain some fascinating surprises. In one dimension the spin 1/2 Heisenberg antiferromagnet can be solved by the Bethe ansatz [11]. The correlation functions have power law decay and there exist local excitations with arbitrarily low energy, i.e., there is no gap in the spectrum immediately above the ground state energy. One might expect similar behavior in any one-dimensional isotropic quantum antiferromagnet. Haldane, however, argued that if the spin is integer then the one-dimensional Heisenberg antiferromagnet has completely different properties [23-25]. He concluded the ground state correlation functions have exponential decay and there is a gap in the spectrum above the ground state energy. There is both experimental and numerical support for these conclusions [12, 16, 36, 37 and references therein].

At first the existence of a gap in the spectrum and exponentially decaying correlation functions is surprising in a model with a continuous symmetry. However, there exist fairly simple models like the Majumdar-Ghosh model [31, 32] which have a translationally invariant Hamiltonian with a continuous symmetry, but have a gap and exponentially (or faster) decaying correlation functions. (Until now there was no proof that this model has a gap, but the proof is supplied in this paper.) In these models, however, the ground state is degenerate and breaks the translational symmetry while the usual Heisenberg antiferromagnet presumably has a unique ground state. The possibility of a quantum antiferromagnet with a continuous symmetry, exponentially decaying correlation functions, a gap and a unique ground state was quite unexpected. In this paper we provide the first rigorous example of a model with all these properties. The model is the one-dimensional spin chain with Hamiltonian

$$
H=\sum_{i}\left[\mathbf{S}_{i} \cdot \mathbf{S}_{i+1}+\frac{1}{3}\left(\mathbf{S}_{i} \cdot \mathbf{S}_{i+1}\right)^{2}\right] .
$$

We prove this model has a unique infinite volume ground state, the correlation functions decay exponentially in this ground state, and there is a gap in the spectrum immediately above the ground state energy. It has been shown rigorously that models like (1.1) do not exhibit such behavior if the spin is halfintegral [5].

In two or more dimensions one generally expects the ground states of quantum antiferromagnets to have Néel order. There is a Goldstone theorem for quantum spin systems which applies to a general class of isotropic Hamiltonians (which includes the usual Heisenberg Hamiltonian and the Hamiltonians we will consider in this paper) [44-46]. It states that if there is Néel order then there is no gap. The existence of Néel order in the ground state can be proven $[21,26]$ for the usual 
Heisenberg Hamiltonian on certain lattices if the spin is large enough, but there may be exceptions. Anderson has argued that the spin 1/2 model on a triangular lattice or a square lattice with a sufficiently strong next nearest neighbor coupling has a ground state without antiferromagnetic order [7]. His "resonating valence bond" (RVB) mechanism is the basis of a possible explanation of high $T_{c}$ superconductors [8]. We provide an example of an isotropic antiferromagnet in two dimensions with spin $3 / 2$ for which we can construct an exact ground state and prove that the two point function decays exponentially in this ground state. We expect that our model has a unique infinite volume ground state and a gap in its spectrum, but we cannot prove either of these conjectures. As in our onedimensional model the Hamiltonian is isotropic, translationally invariant and can be written as a sum over nearest neighbor pairs $i, j$ of polynomials in $\mathbf{S}_{i} \cdot \mathbf{S}_{j}$.

The construction of a ground state and proof of exponential decay of the two point function for our two-dimensional model can be adapted to any bipartite lattice with coordination number three. Since such lattices exist in any dimension greater than one, we expect that in any dimension greater than one there exist models with all the properties that Haldane predicts for the one-dimensional Heisenberg antiferromagnet with integer spin. It should be emphasized that in dimensions greater than one it is not necessary that the spin be integral for this to happen. The relevant criterion appears to involve the lattice type (especially the coordination number), the spin and the dimension.

Our one-dimensional model (1.1) is a special case of the Hamiltonian

$$
H=\sum_{i}\left[\mathbf{S}_{i} \cdot \mathbf{S}_{i+1}-\beta\left(\mathbf{S}_{i} \cdot \mathbf{S}_{i+1}\right)^{2}\right] .
$$

The model with $\beta=0$ is, of course, the standard Heisenberg model which is believed to have a unique massive ground state according to Haldane. We now know rigorously that such a unique massive ground state exists for $\beta=-1 / 3$. The model with $\beta=1$, however, has been solved by the Bethe ansatz method $[9,10,28$, $29,42]$. It has a unique ground state with no energy gap, and appears to have power law decay of the correlation functions by standard field theory arguments. We expect that the model (1.2) has a unique massive ground state for $-1<\beta<1$ and undergoes a phase transition at $\beta=1$ to a dimerized phase with two ground states and a gap for $\beta>1$. At $\beta=-1$ the model has an exact $S U(3)$ symmetry and is very likely massless [3]. For more details and support for this picture we refer the reader to [1] and Sect. 4.4. Finite chain calculations supporting this picture may be found in $[13,20,34,41]$. A critical theory for the phase transition at $\beta=1$ based on the nonabelian bosonization method is given in $[3,4]$.

The possibility of an isotropic model with a unique massive ground state may be understood as follows. For a quantum antiferromagnet the quantum fluctuations always provide some "entropy," even in the ground state. Thus the ground state of a quantum antiferromagnet is like a classical model at some finite temperature. If this classical model is in a high temperature phase then the ground state of the quantum model will be unique and have a finite correlation length, i.e., a mass.

The key to all our models is the idea of a valence bond. Given two spin 1/2's, a valence bond is formed by putting them in the singlet state $\uparrow \downarrow-\downarrow \uparrow$. Now consider 
the spin 1 chain. Each spin 1 can be regarded as the symmetric part of the product of two spin 1/2's. We construct a state with a valence bond between each pair of adjacent sites $i$ and $i+1$ by forming a singlet out of one of the spin 1/2's at site $i$ and one at site $i+1$. After doing this we must symmetrize the two spin 1/2's at each site in order to restore spin 1 at each site. This state can be represented diagramatically as in Fig. 2.1. We call the resulting state a valence bond solid (VBS) state since the valence bond structure is identical to the underlying lattice. We will show in Sect. 2 that this state is a ground state of (1.1). This state was first introduced, to the best of our knowledge, in the discussion of the large $n$ limit of $S U(n)$ chains [2]. Valence bonds have been used before to construct exact ground states [17, 18, 27, 38]. In these previous models, however, the ground states are at least doubly degenerate. Furthermore, these ground states can be written as a single tensor product of states, each of which only involves a few lattice sites. The VBS state cannot be written as such a tensor product.

Such VBS states can be constructed whenever the spin $s$ equals $1 / 2$ of the coordination number, $z$, of the lattice. A generalized VBS state can be constructed whenever $s$ is an integer multiple of $z / 2$ (see Sect. 6 for details). Hamiltonians for which these states are ground states can also be constructed. In one dimension the VBS states can be constructed if and only if the spin is integral. Thus these solvable models provide a qualitative explanation of why integer and half-integer spin chains should have such radically different behavior [2].

In two or more dimensions there are many VBS states because of the freedom at the boundary. We conjecture that if the coordination number $z$ and dimension $d$ are small enough then in the infinite volume limit there is a unique ground state and this state has exponentially decaying correlation functions and a gap. If $z$ and $d$ are large then we conjecture there is Néel order, no gap and infinitely many infinite volume ground states.

While we have no rigorous results on the nonzero temperature behavior of these models in two and more dimensions it is interesting to speculate on what happens. If there is a unique ground state and a gap above the energy of this ground state, then one would expect that for any temperature there is a unique Gibbs state and the correlation length remains bounded as the temperature goes to zero. In other words, it appears that in any dimension there exist isotropic quantum antiferromagnets which do not have a phase transition even at zero temperature.

The organization of the paper is as follows. Section 2 is devoted to the onedimensional model (1.1). The exact ground state is constructed in detail and the two point function is calculated in this ground state. The existence of a gap, the uniqueness of the ground state and the exponential decay of all truncated correlation functions is proven. Our spin $3 / 2$ model on a two-dimensional hexagonal lattice is defined and studied in Sect. 3. A ground state is constructed, and we prove that with periodic boundary conditions the two point function of the ground state has exponential decay. In Sect. 4 we construct the VBS state for a general lattice and speculate on how its properties depend on the dimension and lattice type. For the VBS state on the Cayley tree we show there is Néel order if the coordination number of the lattice exceeds four, but no Néel order when the coordination number equals three. We also discuss some $S U(n)$ models for which 
we can find exact ground states which are generalizations of the VBS state, and develop a random loop representation for these models. The Majumdar-Ghosh model is considered in Sect. 5. This model is known to have two ground states. We prove that these are the only two ground states in the infinite volume limit, and that there is a gap. In Sect. 6 we briefly discuss various generalizations of our models and the Majumdar-Ghosh model. In Appendix A we sketch how the standard technique of Gaussian domination shows that there is Néel order in the ground state of the usual Heisenberg antiferromagnet on the two-dimensional hexagonal lattice if the spin is at least $3 / 2$.

The various sections of this paper are almost independent, so it should be possible to read any one section after a quick look at the preceding sections. Most of the results in this paper were announced in [1].

\section{The One-Dimensional Model (1.1)}

\subsection{The Ground State}

We define our one-dimensional system, and describe its exact ground states in some detail in this subsection. The calculation of the ground state correlation functions, the proof of the existence of an energy gap, and the proof of the uniqueness of the ground state will be given in the following subsections.

We denote sites in our one-dimensional lattice by $i$ and denote the spin operator for spin $s=1$ at site $i$ by $\mathbf{S}_{i}=\left(S^{1}{ }_{i}, S^{2}{ }_{i}, S^{3}\right)$. The restriction of a state to two adjacent sites $i$ and $i+1$ can have spin 0,1 or 2 . We denote the orthogonal projection onto states with spin 2 by $P_{2}\left(\mathbf{S}_{i}+\mathbf{S}_{i+1}\right)$. We can express this projection in terms of the spin operators as follows.

$$
P_{2}\left(\mathbf{S}_{i}+\mathbf{S}_{i+1}\right)=\mathbf{S}_{i} \cdot \mathbf{S}_{i+1} / 2+\left(\mathbf{S}_{i} \cdot \mathbf{S}_{i+1}\right)^{2} / 6+1 / 3 .
$$

The Hamiltonian is the sum over $i$ of these projections.

$$
H=\sum_{i} H_{i} \quad \text { with } \quad H_{i}=P_{2}\left(S_{i}+S_{i+1}\right) .
$$

Obviously $H \geqq 0$, so if we could find a state $\Omega$ with $H_{i} \Omega=0$ for all $i$ then $\Omega$ would be a ground state. Naively, one might not expect such a state to exist, but we will show that in fact it does.

To describe the exact ground states of the above Hamiltonian, we will introduce a special basis for the state space. First, consider the state space for a single spin $1 / 2$. Let $\psi_{1}, \psi_{2}$ denote the eigenstates of $S^{3}$ with eigenvalues $1 / 2$ and $-1 / 2$. The state space for spin 1 may be formed by taking the symmetric part of the tensor product of two spin 1/2 spaces. Thus an orthogonal basis is $\psi_{11}, \psi_{12}=\psi_{21}, \psi_{22}$, where

$$
\psi_{\alpha \beta}=\left[\psi_{\alpha} \otimes \psi_{\beta}+\psi_{\beta} \otimes \psi_{\alpha}\right] / \sqrt{2} .
$$

We emphasize that in this notation $\psi_{\alpha \beta}$ and $\psi_{\beta \alpha}$ denote the same state. These states do not all have norm 1 . Their norms and overlaps are

$$
\left(\psi_{\alpha \beta}, \psi_{\gamma \delta}\right)=\delta_{\alpha \gamma} \delta_{\beta \delta}+\delta_{\alpha \delta} \delta_{\beta \gamma} .
$$


These states may be written in terms of a more standard basis for spin 1 as follows. Let $(+),(0)$, and $(-)$ denote the orthonormal basis for spin 1 consisting of eigenstates of $S^{3}$ with eigenvalues $+1,0$, and -1 , respectively. Then

$$
\psi_{11}=\sqrt{2}(+), \quad \psi_{12}=\psi_{21}=(0), \quad \psi_{22}=\sqrt{2}(-) .
$$

Equation (2.4) hides the $S U(2)$ invariance of the theory. To make the invariance more explicit we should raise the indices of the state $\psi_{\alpha \beta}$ in the left side of the inner product. We accomplish this by adopting the physicists' notation $\psi^{\dagger \alpha \beta} \cdot \psi_{\gamma \delta}$ for the inner product $\left(\psi_{\alpha \beta}, \psi_{\gamma \delta}\right)$. Then (2.4) becomes

$$
\psi^{\dagger \alpha \beta} \cdot \psi_{\gamma \delta}=\delta^{\alpha}{ }_{\gamma} \delta^{\beta}{ }_{\delta}+\delta^{\alpha}{ }_{\delta} \delta_{\gamma}^{\beta}{ }_{\gamma} .
$$

Both $\delta_{\alpha \gamma}$ and $\delta^{\alpha}{ }_{\gamma}$ equal 1 if $\alpha=\gamma$ and 0 otherwise.

There are four spin 1/2's associated with each bond on the chain. If two of these spin $1 / 2$ 's are in a singlet state, i.e., a state with total spin 0 , then the four spin $1 / 2$ 's can only have total spin 0 or 1 . For two spin $1 / 2$ 's, a singlet pair is formed by contracting with an $\varepsilon$ tensor, i.e., $\psi_{\alpha} \otimes \psi_{\beta} \varepsilon^{\alpha \beta}$ is a singlet. $\left(\varepsilon^{\alpha \beta}\right.$ is the antisymmetric tensor with $\varepsilon^{12}=1$.) In this expression and throughout the paper we adopt the convention that repeated upper and lower indices are summed. Given two spin 1's we can form $\Omega_{\alpha \beta}=\psi_{\alpha \gamma} \otimes \psi_{\delta \beta} \varepsilon^{\gamma \delta}$. Since two spin 1/2's are always in a singlet state in $\Omega_{\alpha \beta}, \Omega_{\alpha \beta}$ is a mixture of states with total spin 0 and 1 . Hence $\Omega_{\alpha \beta}$ is a ground state (for two sites) of the projection onto spin 2.

It is now clear how we can form a ground state for a finite chain $\{1,2, \ldots, L\}$. For convenience we take $L$ odd, but the definitions are essentially identical for even L. Let

$$
\Omega_{\alpha \beta}=\psi_{\alpha \beta_{1}} \otimes \psi_{\alpha_{2} \beta_{2}} \otimes \ldots \otimes \psi_{\alpha_{L} \beta} \varepsilon^{\beta_{1} \alpha_{2}} \varepsilon^{\beta_{2} \alpha_{3}} \ldots \varepsilon^{\beta_{L-1} \alpha_{L}} .
$$

For any two adjacent sites $i$ and $i+1$, there is a spin $1 / 2$ at site $i$ and a spin $1 / 2$ at site $i+1$ which are contracted with an $\varepsilon$ tensor to form a singlet. Thus when $\Omega_{\alpha \beta}$ is restricted to sites $i$ and $i+1$, it has only spin 0 and 1 . Hence $H_{i} \Omega_{\alpha \beta}=0$, so $\Omega_{\alpha \beta}$ is a ground state of $H$.

We can simplify our notation by introducing a raising and lowering convention for indices and a shorthand notation for the tensor products. For spin $1 / 2$ states we define $\psi^{\alpha}=\varepsilon^{\alpha \beta} \psi_{\beta}$ with the convention that repeated indices are summed. A singlet pair of spin 1/2's is then formed by contracting an upper and lower index, i.e., $\psi_{\alpha} \otimes \psi^{\alpha}$. For a single spin 1 we define

$$
\psi^{\alpha \beta}=\varepsilon^{\alpha \gamma} \varepsilon^{\beta \delta} \psi_{\gamma \delta} .
$$

It is easily checked that

$$
\left(\psi^{\alpha \beta}, \psi^{\gamma \delta}\right)=\psi_{\alpha \beta}^{\dagger} \cdot \psi^{\gamma \delta}=\delta_{\alpha}^{\gamma} \delta_{\beta}^{\delta}+\delta_{\beta}^{\gamma} \delta_{\alpha}^{\delta} .
$$

For our chain of spin 1's we raise the indices on every other site and introduce the shorthand,

$$
\psi_{\alpha_{1} \beta_{1}}{ }^{\alpha_{2} \beta_{2} \ldots \alpha_{L-1} \beta_{L-1}}{ }_{\alpha_{L} \beta_{L}}=\psi_{\alpha_{1} \beta_{1}} \otimes \psi^{\alpha_{2} \beta_{2}} \otimes \ldots \otimes \psi^{\alpha_{L-1} \beta_{L-1}} \otimes \psi_{\alpha_{L} \beta_{L}} .
$$

Then our ground state (2.7) is compactly written as

$$
\Omega_{\alpha \beta}=\psi_{\alpha \alpha_{1}}^{\alpha_{1} \alpha_{2}{ }_{\alpha_{2} \alpha_{3}} \cdots \alpha_{L-2} \alpha_{L-1}{ }_{\alpha_{L-1} \beta} .}
$$




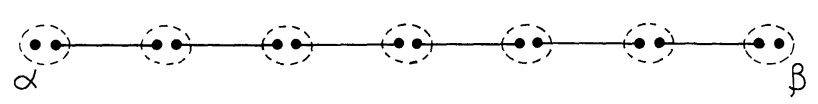

Fig. 2.1. The VBS state $\Omega_{\alpha \beta}$ on a finite chain. Each dot, line, and dotted circle represents a spin $1 / 2$, a singlet pair, and the symmetrization of two spin $1 / 2$ 's to create a spin 1

We can also define the periodic extension of $H$ in (2.2) by adding $P_{2}\left(S_{1}+S_{L}\right)$ to $H$. This is also known as periodic boundary conditions. If $L$ is odd then $\Omega=\Omega_{\alpha \beta} \varepsilon^{\alpha \beta}$ is a ground state. If $L$ is even then $\Omega=\Omega_{\alpha}{ }^{\alpha}$ is a ground state, where $\Omega_{\alpha}{ }^{\beta}$ is the ground state given by the analog of (2.11) for even $L$.

There is a simple diagramatic representation of these VBS states. Each site in the chain is represented by two dots which represent the two spin 1/2's at that site. Each dot has a line coming out of it. Connecting two of these lines means that the two spin 1/2's are put in the singlet state. After forming these singlets we must symmetrize the two dots at each site. This symmetrization is represented by a dotted circle around the two dots. For an open chain there are free lines at each end of the chain corresponding to the indices $\alpha$ and $\beta$ in $\Omega_{\alpha \beta}$. The resulting diagram for the ground state of an open chain is shown in Fig. 2.1.

We refer to these $\Omega_{\alpha \beta}$ and their (unique) infinite volume limit as Valence-Bond Solid (VBS) ground states. This terminology comes from the observation that, in these states, the valence bond (or singlet pair) structure exactly mimics the bond structure of the basic lattice. In Sects. 3, 4, and 6 we will discuss generalizations of VBS states to other lattices.

The VBS ground states appear very simple and natural in the present basis, but these states are not as trivial as they may appear. In particular, these states cannot be written as a single tensor product of a state at each site. To emphasize the richness of these states we will express them in terms of the usual $\{(+),(0),(-)\}$ basis of $S^{3}$ eigenstates at each site. The basis vectors for the chain are labelled by strings of 0's, +'s, and -'s. We denote such strings by $A$ and the corresponding state by $\psi_{A}$. The coefficient of $\psi_{A}$ in the ground state $\Omega_{\alpha \beta}$ will be denoted by $\Omega_{\alpha \beta}(A)$, so $\Omega_{\alpha \beta}=\sum_{A} \Omega_{\alpha \beta}(A) \psi_{A}$.

The coefficient $\Omega_{\alpha \beta}(A)$ is zero unless $A$ is of a special form which depends on $\alpha$ and $\beta$. The rules are as follows:

$\alpha=1, \beta=2: A$ must contain the same number of + 's and -'s. The first nonzero character in $A$ must be a + , and thereafter the nonzero characters must alternate between - and + .

$\alpha=2, \beta=1$ : Same as above with + and - reversed.

$\alpha=1, \beta=1: A$ must contain one more + than - . The first nonzero character must be a + , and thereafter the nonzero characters must alternate between and + .

$\alpha=2, \beta=2$ : Same as above with + and - reversed.

An example of an $A$ in the $\alpha=1, \beta=2$ class is $0+00-+0-0+000-$.

These four classes are disjoint with one exception; the string containing all 0's belongs to both the $\alpha=1, \beta=2$ class and the $\alpha=2, \beta=1$ class. Note that for any 
choice of $\alpha$ and $\beta, \Omega_{\alpha \beta}(A)=0$ if $A$ contains two +'s separated only by 0's or two -'s separated only by 0 's.

We will give an explicit formula for $\Omega_{12}(A)$ and leave the other cases to the reader. Let $A$ be a configuration in the class $\alpha=1, \beta=2$. Let $k$ be the number of pairs of + and - . Let $m$ be the number of odd sites at which there is a + or a - . Then

$$
\Omega_{12}(A)=(-1)^{m} 2^{k} .
$$

To show that this is a ground state, consider two adjacent sites $i$ and $i+1$. It suffices to show that $\Omega_{12}$ is orthogonal to any state which has spin 2 on the pair of sites $i$ and $i+1$. A basis for these spin 2 states is

$$
\begin{aligned}
& e_{1}=(++), \\
& e_{2}=(+0)+(0+), \\
& e_{3}=2(00)+(+-)+(-+), \\
& e_{4}=(-0)+(0-), \\
& e_{5}=(--) .
\end{aligned}
$$

Obviously, $\Omega_{12}$ is orthogonal to $e_{1}$ and $e_{5}$. To see that $\Omega_{12}$ is orthogonal to $e_{2}$, let $A$ be a string with + at site $i$ and 0 at site $i+1$. Let $A^{\prime}$ be the same string except that sites $i$ and $i+1$ are interchanged. This switch leaves $k$ unchanged but changes $m$ by 1. Thus Eq. (2.12) implies that $\Omega_{12}(A)=-\Omega_{12}\left(A^{\prime}\right)$. So $\Omega_{12}$ is orthogonal to $e_{2}$, and, by the same argument, to $e_{4}$. Finally, to see that $\Omega_{12}$ is orthogonal to $e_{3}$, let $A$ be a configuration with 0 's at $i$ and $i+1$. Let $A_{1}$ and $A_{2}$ be the two configurations obtained by replacing the two 0's by -+ and +- , respectively. Exactly one of $A_{1}$ and $A_{2}$ will have a nonzero coefficient. (Which one depends on $\alpha$ and $\beta$ and on what $A$ looks like off of sites $i$ and $i+1$.) Assume $A_{1}$ has the nonzero coefficient. Then Eq. (2.12) yields $\Omega_{12}\left(A_{1}\right)=-2 \Omega_{12}(A)$. It follows that $\Omega_{12}$ is orthogonal to $e_{3}$. Thus Eq. (2.12) defines a ground state.

Remarks. 1. For a finite chain with open boundary conditions we have found four ground states. In Sect. 2.2 we will calculate the norms and overlaps of these four states (2.14). It follows immediately from this calculation that the four ground states are nonzero and linearly independent. In Lemma 2.8 of Sect. 2.4 we will prove that they are the only finite volume ground states. Hence the open chain has a fourfold degenerate ground state. The total spin commutes with the Hamiltonian, so our four ground states must either consist of a triplet of spin 1 states and one spin 0 state or of four spin 0 states. The states $\Omega_{12}, \Omega_{21}, \Omega_{11}, \Omega_{22}$ have total $S^{3}$ equal to $0,0,+1,-1$, respectively. Hence we must have three spin 1 states $\left(\Omega_{11}\right.$, $\Omega_{22}$, and a linear combination of $\Omega_{12}$ and $\Omega_{21}$ ) and one spin 0 state (another linear combination of $\Omega_{12}$ and $\Omega_{21}$ ). It is worth remarking that in the usual Heisenberg antiferromagnet the ground state for any $L$ with open boundary conditions is always nondegenerate and has total spin 0 [30].

2. We can use Remark 1 to show that the periodic chain has a unique ground state. Suppose the periodic chain has a ground state with total spin 1 . Then we can find a ground state for the periodic chain with total $S^{3}$ equal to +1 . Any ground 
state for the periodic chain is also a ground state for the open chain, so this ground state must be a multiple of $\Omega_{11}$. From the description of $\Omega_{11}$ in terms of 0 's, +'s, and -'s, we see that $\Omega_{11}$ contains configurations with + 's at both sites 1 and $L$. Such a configuration, however, has spin 2 when restricted to sites 1 and $L$, and so is not a ground state for the periodic chain. This contradiction shows that the periodic chain only has ground states with total spin 0 . The open chain has only one such ground state, so the periodic chain has a unique ground state.

3. In Sect. 2.4 we will show that the four ground states all yield the same state in the infinite volume limit and that the resulting infinite volume state is the only infinite volume ground state. In particular, the infinite volume limit constructed using periodic boundary conditions equals the infinite volume limit constructed using open boundary conditions.

\subsection{The Ground State Two-Point Correlation Function}

In this subsection we will calculate the two point correlation function for the ground state. By Remark 3 above, we need only perform the calculation for a particular choice of boundary conditions. We will use periodic boundary conditions. We will show that

$$
\omega\left(S^{a}{ }_{0} S_{r}^{b}\right)=\delta^{a b}(-1)^{r}(4 / 3) 3^{-r},
$$

where $\omega\left(S^{a}{ }_{0} S^{b}{ }_{r}\right)$ denotes the limit as $L \rightarrow \infty$ of the expectation of $S^{a}{ }_{0} S^{b}{ }_{r}$ in the ground state of the chain with $L$ sites and periodic boundary conditions.

We begin by calculating the normalization of the ground state for an open chain of $L$ sites. For later use we will actually calculate the inner product of any two of our four ground states. Since the notation depends on whether $L$ is even or odd we assume that $L$ is even. The basic formulae needed for this calculation are Eqs. (2.6) and (2.9). The calculation may be done in a diagramatic fashion using the diagrams introduced in Fig. 2.1.

We draw the state $\Omega$ twice, but label the two drawings as $\Omega$ and $\Omega^{\dagger}$ as shown in Fig. 2.2a. At each site the inner product is, according to (2.6) or (2.9), a sum of two terms. We represent them by Fig. 2.2b. Inserting Fig. 2.2b at each site in Fig. 2.2a gives a sum of $2^{L}$ diagrams. For example, with two sites the inner product is given by Fig. 2.2c. Each loop represents the trace of a product of $\delta$ tensors and so gives a factor of $\delta_{\alpha}^{\alpha}=2$. Thus Fig. 2.2c yields

$$
\begin{aligned}
\Omega^{\dagger \alpha}{ }_{\beta} \cdot \Omega_{\gamma}{ }^{\delta} & =2 \delta^{\alpha}{ }_{\gamma} \delta_{\beta}{ }_{\beta}+\delta^{\alpha}{ }_{\gamma} \delta^{\delta}{ }_{\beta}+\delta^{\alpha}{ }_{\gamma} \delta^{\delta}{ }_{\beta}+\delta^{\alpha}{ }_{\beta} \delta^{\delta}{ }_{\gamma} \\
& =4 \delta^{\alpha}{ }_{\gamma} \delta^{\delta}{ }_{\beta}+\delta^{\alpha}{ }_{\beta} \delta^{\delta}{ }_{\gamma} .
\end{aligned}
$$
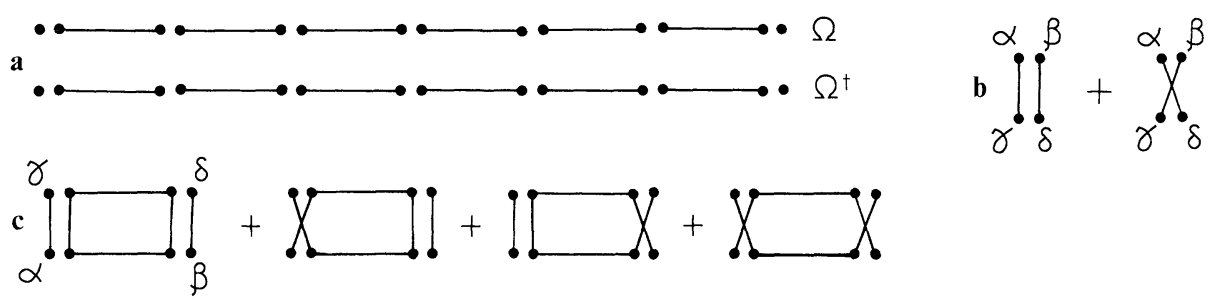

Fig. 2.2a-b. Inserting $\mathbf{b}$ into each site in a gives the overlap $\Omega^{\dagger} \cdot \Omega$. $\mathbf{c}$ The case with two sites 


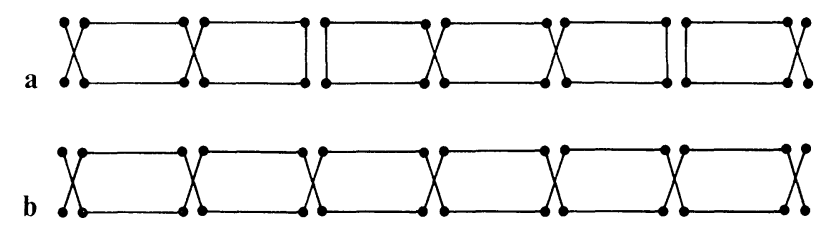

Fig. 2.3. Diagrams which appear in the overlap calculation. a A typical one, and $\mathbf{b}$ the special one has no loops

For general even $L$ all but one diagram consists of an open line from $\alpha$ to $\gamma$, an open line from $\beta$ to $\delta$ and some number of closed loops in between. A typical diagram is shown in Fig. 2.3a. All these diagrams give a contribution proportional to $\delta^{\alpha}{ }_{\gamma} \delta_{\beta}^{\delta}$. The one other diagram consists of two lines stretching all the way across the lattice (Fig. 2.3b) and gives simply $\delta^{\alpha}{ }_{\beta} \delta^{\delta}{ }_{\gamma}$. Thus we need to perform the sum over all loop diagrams. For each loop we get a factor of 2 from $\delta_{\alpha}^{\alpha}=2$. The number of diagrams with $m$ loops is the number of ways of choosing the $(m+1)$ points where the loops begin and end out of the $L$ possible points, i.e., it is given by the binomial coefficient $\left(\begin{array}{c}L \\ m+1\end{array}\right)$. Thus the sum of all diagrams of this type is proportional to

$$
\sum_{m=0}^{L-1}\left(\begin{array}{c}
L \\
m+1
\end{array}\right) 2^{m}=\left[(2+1)^{L}-1\right] / 2
$$

and so

$$
\Omega^{\dagger \alpha}{ }_{\beta} \cdot \Omega_{\gamma}{ }^{\delta}=\delta^{\alpha}{ }_{\gamma} \delta^{\delta}{ }_{\beta}\left(3^{L}-1\right) / 2+\delta^{\alpha}{ }_{\beta} \delta^{\delta}{ }_{\gamma} .
$$

If $L$ is odd a similar computation yields

$$
\Omega^{\dagger \alpha \beta} \cdot \Omega_{\gamma \delta}=\delta^{\alpha}{ }_{\gamma} \delta^{\beta}{ }_{\delta}\left(3^{L}-1\right) / 2+\delta^{\alpha}{ }_{\delta} \delta^{\beta}{ }_{\gamma} .
$$

The normalization of the ground state with periodic boundary conditions

$$
\Omega_{\beta}^{\dagger \beta} \cdot \Omega_{\alpha}^{\alpha}=3^{L}+3,
$$

can be found by taking traces in (2.14) or by summing graphs on a circle.

Next we calculate the spin-spin correlation function for a chain with $L$ sites and periodic boundary conditions. The spin operators acting on our basis for a single spin 1 give

$$
\mathbf{S} \psi_{\alpha \beta}=-(1 / 2) \boldsymbol{\sigma}_{\alpha}{ }^{\gamma} \psi_{\gamma \beta}-(1 / 2) \boldsymbol{\sigma}_{\beta}^{\gamma} \psi_{\alpha \gamma}
$$

on odd sites, or

$$
\mathbf{S} \psi^{\alpha \beta}=(1 / 2) \boldsymbol{\sigma}_{\gamma}^{\alpha} \psi^{\gamma \beta}+(1 / 2) \boldsymbol{\sigma}^{\beta}{ }_{\gamma} \psi^{\alpha \gamma}
$$

on even sites, where the $\left(\sigma^{a}\right)_{\beta}^{\alpha}$ 's are the Pauli matrices and

$$
\boldsymbol{\sigma}_{\beta}^{\alpha}=\varepsilon^{\alpha \gamma} \varepsilon_{\beta \delta} \boldsymbol{\sigma}_{\gamma}{ }^{\delta} .
$$

Thus acting with $\mathbf{S}_{\mathbf{r}}$ on the periodic ground state, breaks either the link between sites $r-1$ and $r$ or between $r$ and $r+1$ and contracts the two dangling indices with 


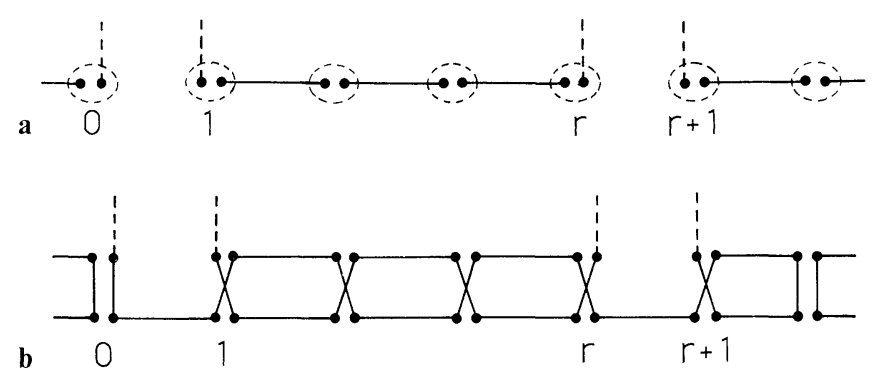

Fig. 2.4. a One of the four diagrams which represent the action of $\mathbf{S}_{0} \cdot \mathbf{S}_{\boldsymbol{r}}$ on $\Omega$. b A typical diagram which gives a nonzero contribution to $\Omega^{\dagger} \cdot \mathbf{S}_{0} \cdot \mathbf{S}_{r} \Omega$

$\pm(1 / 2) \boldsymbol{\sigma}$ (for $r$ even or odd). Acting with $S^{a}{ }_{0} S^{b}{ }_{r}$ (we assume $r>2$ ) breaks the chain up into two open lines, one running from 0 or 1 to $r-1$ or $r$ and the other running from $r$ or $r+1$ to $L$ or 0 . One of these four cases is shown in Fig. 2.4a. The dangling indices near 0 are contracted with $(1 / 2) \sigma^{a}$ and those near $r$ with $(-1)^{r}(1 / 2) \sigma^{b}$.

To obtain the correlation function we need to calculate the overlap of this state with the ground state. Again we represent the calculation by a sum of diagrams. There now must be two lines which end on the four dangling bonds, all others forming closed loops. These two lines can both stretch from 0 to $r( \pm 1)$ or one can start and end near 0 , and the other start and end near $r$. There are far more diagrams of the latter type, however, they all give contributions proportional to $\delta^{\alpha}{ }_{\gamma}\left(\sigma^{a}\right)_{\alpha}^{\gamma} \delta^{\beta}{ }_{\delta}\left(\sigma^{b}\right)_{\beta}{ }^{\delta}=\operatorname{Tr} \sigma^{a} \operatorname{Tr} \sigma^{b}=0$. The former diagrams give contributions proportional to $\left(\sigma^{a}\right)_{\alpha}{ }^{\gamma} \delta_{\gamma}{ }^{\beta}\left(\sigma^{b}\right)_{\beta}{ }^{\delta} \delta_{\delta}{ }^{\alpha}=\operatorname{Tr} \sigma^{a} \sigma^{b}=2 \delta^{a b}$. The lines from 0 to $r$ can run around the chain in either direction but for $L \rightarrow \infty$ with $r$ fixed they must take the shorter path to contribute. Apart from these two lines there are one or more closed loops (many as $L \rightarrow \infty$ ) going around the chain the long way (see Fig. 2.4b). Summing over all these diagrams as before, being careful to sum over the four possible combinations of broken bonds and then dividing by the ground state normalization, we obtain (2.13).

\subsection{The Energy Gap}

We will prove that the Hamiltonian (2.2) has a gap between the ground state energy and the first excited state. In this subsection we begin by showing that for a finite chain of length $L$ the gap is bounded away from zero as $L \rightarrow \infty$. In Sect. 2.4 we will show that the infinite volume system has a gap.

We use an open chain and assume that the four ground states we constructed in the previous section are the only ground states. We will prove they are the only ground states in Sect. 2.4. Bounding the gap away from zero uniformly in $L$ is equivalent to the following theorem.

Theorem 2.1. Consider the Hamiltonian

$$
H_{1, L}=\sum_{i=1}^{L-1} H_{i}
$$


There is a positive constant $\varepsilon$ which does not depend on $L$ such that

$$
\left(\psi, H_{1, L} \psi\right) \geqq \varepsilon(\psi, \psi)
$$

for all $\psi$ which are orthogonal to the four ground states of $H_{1, L}$.

Proof. Our proof will take advantage of the following property of our model. If $\Omega$ is a ground state then for each $i,\left(\Omega, H_{i} \Omega\right)$ equals the lowest eigenvalue of $H_{i}$. Consequently, any ground state for $H_{1, n}$ is also a ground state for $H_{1, n-1}$.

Let $Q_{n}$ be the orthogonal projection onto the subspace of states which are ground states for $H_{1, n}, n \leqq L$. Let $P_{n}=1-Q_{n}$. The previous paragraph implies $Q_{n} \geqq Q_{n+1}$, so $P_{n} \leqq P_{n+1}$. Inequality (2.17) is equivalent to $H_{1, L} \geqq \varepsilon P_{L}$. We write $P_{L}$ as a sum of mutually orthogonal projections:

$$
P_{L}=\sum_{n=l}^{L-1}\left(P_{n+1}-P_{n}\right)+P_{l} .
$$

The integer $l$ will be chosen later. It will be independent of $L$. (We assume, of course, that $L>l$.)

The idea of the proof is to bound $P_{n+1}-P_{n}=Q_{n}-Q_{n+1}$ by the terms $H_{i}$ in the Hamiltonian with $i$ near $n$. If we restrict $Q_{n}-Q_{n+1}$ to the first $n+1$ sites, then it is an 8-dimensional projection. $\left(Q_{n}\right.$ is 12-dimensional and $Q_{n+1}$ is 4-dimensional.) Let $\varphi_{n+1}^{i}, i=1, \ldots, 8$ be states on the first $n+1$ sites which are an orthonormal basis for the range of this projection. Each $\varphi_{n+1}^{i}$ is a ground state for $H_{1, n}$ and is orthogonal to any ground state for $H_{1, n+1}$.

Let $H_{n-l+1, n+1}$ be the Hamiltonian for sites $n-l+1$ to $n+1$, and let $Q_{n-l+1, n+1}$ be the orthogonal projection onto its ground states. Define

$$
\psi_{n+1}^{i}=Q_{n-l+1, n+1} \varphi_{n+1}^{i} /\left\|Q_{n-l+1, n+1} \varphi_{n+1}^{i}\right\|
$$

and define

$$
\varepsilon(l)=\sup _{n} \max _{i}\left\|Q_{n-l+1, n+1} \varphi_{n+1}^{i}\right\|^{2} .
$$

We will reduce the proof to the following three lemmas.

Lemma 2.2. Let $e_{l+1}$ be the gap for $H_{1, l+1}$, i.e., the first nonzero eigenvalue of $H_{1, l+1}$. Then

$$
Q_{n}-Q_{n+1} \leqq 2 \varepsilon(l) \sum_{i=1}^{8} P\left(\psi_{n+1}^{i}\right)+\frac{2}{e_{l+1}} H_{n-l+1, n+1},
$$

where $P\left(\psi_{n+1}^{i}\right)$ is the orthogonal projection onto the states of the form $\psi_{n+1}^{i} \otimes \chi$ for any state $\chi$ on sites $n+2$ to $L$.

Lemma 2.3. If $|m-n| \geqq l+1$ then for any $i$ and $j, P\left(\psi_{n+1}^{i}\right)$ is orthogonal to $P\left(\psi_{m+1}^{j}\right)$, i.e., $P\left(\psi_{n+1}^{i}\right) P\left(\psi_{m+1}^{j}\right)=0$.

Lemma 2.4. There is a constant $c$ such that for all $l$

$$
\varepsilon(l) \leqq c 3^{-l} .
$$


Assuming the three lemmas we complete the proof as follows. We will sum (2.18) from $n=l$ to $L-1$. Note that

$$
\begin{gathered}
\sum_{n=l}^{L-1}\left(Q_{n}-Q_{n+1}\right)=Q_{l}-Q_{L}=P_{L}-P_{l}, \\
\sum_{n=l}^{L-1} H_{n-l+1, n+1} \leqq(l+1) H_{1, L} .
\end{gathered}
$$

In the sum

$$
\sum_{n=l}^{L-1} \sum_{i=1}^{8} P\left(\psi_{n+1}^{i}\right)
$$

any two terms with $n=n_{1}$ and $n=n_{2}$ are orthogonal if $\left|n_{1}-n_{2}\right|>l$. Since any sum of mutually orthogonal projections is less than or equal to the identity operator, $(2.22)$ is less than or equal to $8(l+1)$. Thus summing $(2.18)$ yields

$$
P_{L}-P_{l} \leqq 16(l+1) \varepsilon(l)+\frac{2(l+1)}{e_{l+1}} H_{1, L} .
$$

Since $e_{l}$ is the first positive eigenvalue of $H_{1, l}$, we trivially have

$$
P_{l} \leqq \frac{1}{e_{l}} H_{1, l} \leqq \frac{1}{e_{l}} H_{1, L} .
$$

Adding the above two inequalities implies

$$
P_{L} \leqq 16(l+1) \varepsilon(l)+\left(\frac{2(l+1)}{e_{l+1}}+\frac{1}{e_{l}}\right) H_{1, L} .
$$

Since $P_{L}$ and $H_{1, L}$ can be simultaneously diagonalized, this implies inequality (2.17) provided $16(l+1) \varepsilon(l)<1$. By Lemma 2.4 we can make this quantity less than 1 by choosing $l$ sufficiently large. Note that we have not assumed anything about how $e_{l}$ depends on $l$. The only information we have used about $e_{l}$ is that it is strictly positive.

Proof of Lemma 2.2. In this proof we will abbreviate $Q_{n-l+1, n+1}$ by $Q$. Each operator in (2.18) acts as the identity on sites $n+2$ to $L$. Thus we may restrict our attention to sites 1 to $n+1$. For any state $\chi$ on sites 1 to $n+1$,

$$
\begin{aligned}
\left(\chi, P\left(\varphi_{n+1}^{i}\right) \chi\right) & =\left|\left(\chi, \varphi_{n+1}^{i}\right)\right|^{2} \\
& =\left|\left(\chi, Q \varphi_{n+1}^{i}+(1-Q) \varphi_{n+1}^{i}\right)\right|^{2} \\
& \leqq 2\left|\left(\chi, Q \varphi_{n+1}^{i}\right)\right|^{2}+2\left|\left(\chi,(1-Q) \varphi_{n+1}^{i}\right)\right|^{2},
\end{aligned}
$$

where we have used the Cauchy-Schwarz inequality to bound the cross terms. The first term equals

$$
2\left\|Q \varphi^{i}{ }_{n+1}\right\|^{2}\left(\chi, P\left(\psi_{n+1}^{i}\right) \chi\right) \leqq 2 \varepsilon(l)\left(\chi, P\left(\psi_{n+1}^{i}\right) \chi\right),
$$


since $\psi_{n+1}^{i}$ is defined as the unit vector proportional to $Q \varphi^{i}{ }_{n+1}$. For the second term the orthonormality of the $\varphi_{n+1}^{i}$ implies

$$
\begin{aligned}
\sum_{i=1}^{8}\left|\left(\chi,(1-Q) \varphi_{n+1}^{i}\right)\right|^{2} & =\sum_{i=1}^{8}\left|\left((1-Q) \chi, \varphi_{n+1}^{i}\right)\right|^{2} \\
& \leqq\|(1-Q) \chi\|^{2} .
\end{aligned}
$$

Thus summing (2.23) from $i=1$ to 8 we obtain

$$
\begin{aligned}
\left(\chi,\left(Q_{n}-Q_{n+1}\right) \chi\right) & =\sum_{i=1}^{8}\left(\chi, P\left(\varphi^{i}{ }_{n+1}\right) \chi\right) \\
& \leqq 2 \varepsilon(l) \sum_{i=1}^{8}\left(\chi, P\left(\psi_{n+1}^{i}\right) \chi\right)+2\|(1-Q) \chi\|^{2} .
\end{aligned}
$$

But $\|(1-Q) \chi\|^{2}=(\chi,(1-Q) \chi)$ and $1-Q \leqq H_{n-l+1, n+1} / e_{l+1}$. The lemma follows.

Proof of Lemma 2.3. In this proof we abbreviate $Q_{n-l+1, n+1}$ and $Q_{m-l+1, m+1}$ by $Q^{n}$ and $Q^{m}$. We can assume $m \geqq n+l+1$. To show the two projections are orthogonal, it suffices to show that the inner product $\left(Q^{n} \varphi^{i}{ }_{n+1} \otimes \chi, Q^{m} \varphi^{j}{ }_{m+1} \otimes \Theta\right)$ vanishes for any state $\chi$ on sites $n+2$ to $L$ and any state $\Theta$ on sites $m+2$ to $L$. We should warn the reader that the two $\otimes$ 's in this inner product mean different things. The first $\otimes$ indicates a tensor product between a state on sites 1 to $n+1$ and a state on sites $n+2$ to $L$. The second $\otimes$ indicates the same thing except that $n$ is replaced by $m$.

Since $Q^{n}$ and $Q^{m}$ commute the inner product equals $\left(\varphi_{n+1}^{i} \otimes \chi\right.$, $\left.Q^{m} Q^{n} \varphi^{j}{ }_{m+1} \otimes \Theta\right)$. But $\varphi^{j}{ }_{m+1}$ is a ground state for $H_{1, m}$ and hence for $H_{n-l+1, n+1}$. So the inner product equals $\left(\varphi_{n+1}^{i} \otimes \chi, Q^{m} \varphi^{j}{ }_{m+1} \otimes \Theta\right)$. Now $\varphi_{m+1}^{j}$ is a ground state for $H_{1, m}$ and so is a ground state for $H_{1, n+1}$. Since $Q^{m}$ acts only on sites greater than $m-1$ and $m-1 \geqq n+1, Q^{m} \varphi^{j}{ }_{m+1} \otimes \Theta$ is also a ground state for $H_{1, n+1}$. But $\varphi^{i}{ }_{n+1}$ is orthogonal to such states, so the inner product vanishes.

Proof of Lemma 2.4. All of the states we will encounter in this proof will be linear combinations of the states shown diagramatically in Fig. 2.5. Our notation for this state depends on whether $n$ and $l$ are even or odd. For brevity we will only consider the case that $n$ and $l$ are both even. In this case the state in Fig. 2.5 is denoted by $\Omega_{\alpha \gamma \varepsilon \varrho}^{\beta \delta}$. The argument in the other three cases is the same.

Let $\varphi$ be one of the states $\varphi_{n+1}^{i}$. Then $\varphi$ is a ground state of $H_{1, n}$ and so is a linear combination of the states $\Omega_{\alpha \beta}^{\beta}{ }^{\delta}{ }_{\varepsilon \varepsilon}$, i.e.,

$$
\varphi=\Omega_{\alpha \beta \varepsilon \varrho}^{\beta \delta} A_{\delta}^{\alpha} \text {. }
$$

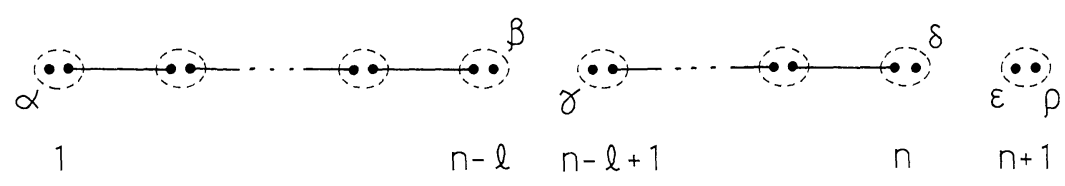

Fig. 2.5. The states $\Omega_{\alpha}{ }^{\beta} \gamma_{\varepsilon}{ }_{\varepsilon}$ which are used in the proof of the existence of a gap 
We can assume $A$ is symmetric in $\varepsilon$ and $\varrho$. Since $Q_{n+1} \varphi=0, \varphi$ is orthogonal to the ground states of $H_{1, n+1}$, i.e., to the states $\Omega_{\mu \gamma \sigma \nu}^{\gamma \sigma}$. Using Eq. (2.14) this implies

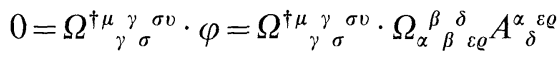

$$
\begin{aligned}
& =\left[\delta^{\mu}{ }_{\alpha} \delta^{\delta}{ }_{\sigma}\left(3^{n}-1\right) / 2+\delta^{\delta}{ }_{\alpha} \delta^{\mu}{ }_{\sigma}\right]\left[\delta^{\alpha}{ }_{\varepsilon} \delta^{v}{ }_{\varrho}+\delta^{\sigma}{ }_{\varrho} \delta^{v}{ }_{\varepsilon}\right] A^{\alpha}{ }_{\delta}{ }^{\varepsilon}{ }^{\mu} \\
& =A_{\delta}^{\mu \nu}\left(3^{n}-1\right)+2 A_{\delta}^{\delta \mu \nu} \text {. }
\end{aligned}
$$

Thus

$$
A_{\delta}^{\mu}{ }_{\delta}^{\delta 0}=-2 A_{\delta}^{\delta}{ }_{\delta}^{\mu v} /\left(3^{n}-1\right)
$$

Thus whenever the second and third indices in $A_{\delta}^{\alpha}{ }_{\delta}^{\varepsilon \varrho}$ are contracted, the result is of order $3^{-n}$ compared with $A_{\delta}^{\alpha}{ }_{\delta}^{\varepsilon}$.

Our goal is to bound the square of the norm of $Q_{n-l+1, n+1} \varphi$. First, we show that this state is a linear combination of the states $\Omega_{\mu}{ }_{\mu}{ }^{\gamma}{ }_{\gamma}{ }_{\gamma}$. To do so we must show that $Q_{n-l+1, n+1} \varphi$ is a ground state of $H_{1, n-l}$ and of $H_{n-l+1, n+1}$. The latter case is trivial since $Q_{n-l+1, n+1}$ is the projection onto the ground states of $H_{n-l+1, n+1}$. The former case is also immediate since $\varphi$ is a ground state of $H_{1, n}$ and so of $H_{1, n-l}$ and $Q_{n-l+1, n+1}$ commutes with $H_{1, n-l}$.

The number of states $\Omega_{\mu{ }^{0}{ }^{\gamma}{ }^{\gamma} \omega}$ is independent of $n$ and $l$, so the preceding paragraph implies that $\left\|Q_{n-l+1, n+1} \varphi\right\|^{2}=\left(Q_{n-l+1, n+1} \varphi, \varphi\right)$ is bounded by a

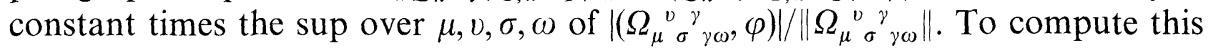
inner product we apply (2.14) twice, once for sites 1 to $n-l$ and once for sites $n-l+1$ to $n$. This yields

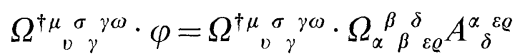

$$
\begin{aligned}
& =\left[\delta^{\mu}{ }_{\alpha} \delta^{\beta}{ }_{\nu}\left(3^{n-l}-1\right) / 2+\delta^{\beta}{ }_{\alpha} \delta^{\mu}{ }_{v}\right]\left[\delta^{\sigma}{ }_{\beta} \delta^{\delta}{ }_{\gamma}\left(3^{l}-1\right) / 2+\delta^{\sigma}{ }_{\gamma} \delta^{\delta}{ }_{\beta}\right] \\
& \times\left[\delta_{\varepsilon}^{\gamma} \delta^{\omega}{ }_{\varrho}+\delta^{\gamma}{ }_{\varrho} \delta^{\omega}{ }_{\varepsilon}\right] A^{\alpha}{ }_{\delta}{ }^{\varepsilon}{ }^{\prime} \\
& =A^{\mu}{ }_{\gamma}{ }^{\gamma \omega} \delta^{\delta}{ }_{v}\left(3^{n-l}-1\right)\left(3^{l}-1\right) / 2+A^{\mu}{ }_{v}{ }^{\sigma \omega}\left(3^{n-l}-1\right) \\
& +A^{\sigma}{ }_{\gamma}{ }^{\gamma \omega} \delta^{\mu}{ }_{v}\left(3^{l}-1\right)+2 A_{\delta}^{\delta \sigma \omega} \delta^{\mu}{ }_{v} \text {. }
\end{aligned}
$$

It is now clear how the proof works. In the first term the second and third indices of $A$ are contracted. By (2.25) this gives us a factor of $3^{-n}$. The other terms in the above sum are smaller than the first term by a factor of $3^{-l}$ or $3^{-n}$. Since $n \geqq l$, the overlap in question is of order $3^{-l}$.

To implement this intuition we need to consider the various normalizations. Recall that $\varphi$ has norm 1 by definition. This implies, after some computation using Eqs. (2.14) and (2.24), that

$$
1=A_{\mu \varepsilon \varrho}^{*} A_{\gamma}^{\mu}{ }_{\gamma}^{\varepsilon Q}\left(3^{n}-1\right) / 2+A_{\delta \varepsilon \varrho}^{* \delta} A_{\mu}^{\mu \varrho} .
$$

$\left(A^{*}{ }_{\mu \varepsilon \varrho}^{\gamma}\right.$ denotes the complex conjugate of $A_{\gamma}^{\mu \varepsilon \varrho}$.) The second term in the right side is nonnegative and the first is just the sum over $\mu, \gamma, \varepsilon$, and $\varrho$ of $\left|A_{\mu}{ }_{\varepsilon \varrho}\right|^{2}\left(3^{n}-1\right) / 2$. Thus

$$
\left|A_{\mu \varepsilon \varrho}^{\gamma}\right|^{2} \leqq 2\left(3^{n}-1\right)^{-1} \text {. }
$$

The norm of $\Omega_{\mu \sigma^{\gamma}{ }^{\gamma} \omega}$ can be computed using (2.14). The result is $3^{n+1} / 4$ plus terms which are smaller by a factor of $3^{-n}$ or $3^{-l}$. Combining this calculation with 
(2.26) and (2.27) shows $\left|\left(\Omega_{\mu \sigma^{\nu} \gamma \omega}^{\gamma}, \varphi\right)\right| /\left\|\Omega_{\mu \sigma^{\gamma} \gamma \omega}{ }^{\gamma}\right\|$ is bounded by a constant times $3^{-l}$.

\subsection{The Infinite Chain}

In this subsection, we prove that the infinite volume system has a unique ground state, exponential decay of all truncated correlation functions in the ground state and a gap. We also prove that the finite volume ground states defined in Sect. 2.1 are the only ones. In this subsection instead of the finite chain $\{1,2, \ldots, L\}$, we use the finite chain $\{-L,-L+1, \ldots, L\}$. The ground states for this finite chain are defined by analogy to (2.11) and denoted by $\Omega^{(L)}{ }_{\alpha \beta}$. The natural way to construct an infinite volume ground state is to compute expectations in $\Omega^{(L)}{ }_{\alpha \beta}$ and then let $L \rightarrow \infty$. Surprisingly, the result of this operation can be exactly expressed in terms of finite volume expectations as we will show in Lemma 2.6.

A local observable, $A$, is any polynomial in the spin operators for finitely many spins. (The identity operator $I$ is a local observable.) If the spins involved in $A$ involve only the sites $\{n, n+1, \ldots, n+m\}$ we say that the support of $A$ is contained in $\{n, n+1, \ldots, n+m\}$. A state $\varrho$ is a function on the local observables such that $\varrho(I)=1, \varrho(\lambda A+\gamma B)=\lambda \varrho(A)+\gamma \varrho(B)$ for all $\lambda, \gamma \in C$ and $\varrho\left(A^{*} A\right) \geqq 0$ for all $A$.

Lemma 2.6. Let $A$ be a local observable. Then for any $l$ such that the support of $A$ is contained in $\{-l,-l+1, \ldots, l\}$,

$$
\lim _{L \rightarrow \infty} \frac{\left(\Omega^{(L)}{ }_{\alpha \beta}, A \Omega^{(L)}{ }_{\alpha \beta}\right)}{\left(\Omega^{(L)}{ }_{\alpha \beta}, \Omega^{(L)}{ }_{\alpha \beta}\right)}=\frac{\sum_{\varepsilon, \delta}\left(\Omega^{(l)}{ }_{\varepsilon \delta}, A \Omega^{(l)}{ }_{\varepsilon \delta}\right)}{\sum_{\varepsilon, \delta}\left(\Omega^{(l)}{ }_{\varepsilon \delta}, \Omega^{(l)}{ }_{\varepsilon \delta}\right)}
$$

for any choice of $\alpha$ and $\beta$. ( $\alpha$ and $\beta$ are not summed over in this equation.) In particular, the limit in the left side of the equation exists and is independent of $\alpha$ and $\beta$. We denote this limit (which is a fortiori a state) by $\omega(A)$.

Proof. In this proof some repeated indices are not summed, so we will explicitly write out all summations. We can write $\Omega_{\alpha \beta}^{(L)}$ as

$$
\Omega_{\alpha \beta}^{(L)}=\sum_{\gamma, \delta} \bar{\Omega}_{\alpha}^{\gamma} \Omega_{\gamma \delta}^{(l)} \widetilde{\Omega}_{\beta}^{\delta},
$$

where $\bar{\Omega}_{\alpha}^{\gamma}, \Omega^{(l)}{ }_{\gamma \delta}$, and $\widetilde{\Omega}^{\delta}{ }_{\beta}$ are ground states on $\{-L, \ldots,-l-1\},\{-l, \ldots, l\}$, and $\{l+1, \ldots, L\}$, respectively. (We are assuming $L-l$ is even. If it is not, some indices must be raised or lowered.) This representation yields

$$
\left(\Omega_{\alpha \beta}^{(L)}, A \Omega_{\alpha \beta}^{(L)}\right)=\sum_{\gamma, \delta, \varepsilon, \varrho}\left(\bar{\Omega}_{\alpha}^{\gamma}, \bar{\Omega}_{\alpha}^{\varepsilon}\right)\left(\Omega_{\gamma \delta}^{(l)}, A \Omega_{\varepsilon \varrho}^{(l)}\right)\left(\widetilde{\Omega}_{\beta}^{\delta}, \widetilde{\Omega}_{\beta}{ }_{\beta}\right) .
$$

By (2.14b),

$$
\begin{aligned}
& \left(\bar{\Omega}_{\alpha}^{\gamma}, \bar{\Omega}_{\alpha}^{\varepsilon}\right)=\delta_{\gamma}^{\varepsilon}\left(3^{L-l}-1\right) / 2+\delta_{\alpha}^{\gamma} \delta_{\alpha}^{\varepsilon}, \\
& \left(\widetilde{\Omega}_{\beta}^{\delta}, \widetilde{\Omega}_{\beta}{ }_{\beta}\right)=\delta_{\delta}{ }_{\delta}\left(3^{L-l}-1\right) / 2+\delta^{\beta}{ }_{\delta} \delta^{\varrho}{ }_{\beta},
\end{aligned}
$$

with no summation over $\alpha$ or $\beta$. Thus

$$
\left(\Omega_{\alpha \beta}^{(L)}, A \Omega_{\alpha \beta}^{(L)}\right)=\left(3^{L-l}-1\right)^{2} / 4 \sum_{\gamma, \delta}\left(\Omega_{\gamma \delta}^{(l)}, A \Omega_{\gamma \delta}^{(l)}\right)+\ldots,
$$


where the three terms represented by $\ldots$ are all smaller by at least a factor of $3^{L-l}$. The lemma now follows.

Next we show that $\omega$ is the only infinite volume ground state.

Theorem 2.7. Assume that a state @ on the infinite chain is ground state in the sense that $\varrho$ satisfies $\varrho\left(H_{i}\right)=0$ for all $i$, where $H_{i}$ is the local Hamiltonian defined in (2.2). Then the state $\varrho$ equals the state $\omega$ defined in the previous lemma.

Remark. Note that the definition of the ground state in the above theorem differs slightly from the "standard" definition in the mathematical physics literature. In the standard definition, the set of ground states $G_{0}$ is

$$
G_{0}=\left\{\varrho \mid \varrho\left(A^{*}[H, A]\right) \geqq 0 \text { for an arbitrary local operator } A\right\},
$$

while our ground state set $G_{1}$ is

$$
G_{1}=\left\{\varrho \mid \varrho\left(H_{i}\right)=E_{0} \text { for all } i\right\},
$$

where $E_{0}$ is the following ground state energy per site:

$$
E_{0}=\inf _{\varrho} \lim _{N \rightarrow \infty}(1 / N) \varrho\left(\sum_{i=-N / 2}^{N / 2} H_{i}\right) \text {. }
$$

Since some models have a state which belongs to $G_{0}$ but not to $G_{1}$ (e.g., the domain wall state in the Ising chain) we cannot expect these two definitions to be equivalent. Using the ergodic theorem [35], we can show that any translation invariant $\varrho$ in $G_{0}$ also belongs to $G_{1}$. When $E_{0}$ happens to be the minimum eigenvalue of the local Hamiltonian $H_{i}$ (as in our model), the standard property [14] of $G_{0}$ implies $G_{1} \subset G_{0}$. In the present model, we expect that the two sets are identical. Another definition of the ground state, which is in many cases equivalent to $G_{0}$, can be found in [6].

The proof of the theorem is based on the lemma above and the following two lemmas.

Lemma 2.8 (Finite volume lemma). Let $\varphi$ be a state for the sites $-L$ to $L$ which satisfies $H_{i} \varphi=0$ for $i=-L, \ldots, L-1$. Then $\varphi$ can be written as

$$
\varphi=A^{\alpha \beta} \Omega_{\alpha \beta}^{(L)},
$$

where $A^{\alpha \beta}(\alpha, \beta=1,2)$ are complex coefficients, and $\Omega^{(L)}{ }_{\alpha \beta}$ are the finite volume ground states defined in (2.11).

Lemma 2.9. Let $A$ be an arbitrary local operator. Then

$$
\lim _{L \rightarrow \infty} \frac{\left(\Omega^{(L)}{ }_{\alpha \beta}, A \Omega^{(L)}{ }_{\gamma \delta}\right)}{\left\|\Omega^{(L)}{ }_{\alpha \beta}\right\|\left\|\Omega^{(L)}{ }_{\gamma \delta}\right\|}=0 \quad \text { (no sum over } \alpha, \beta, \gamma \text { or } \delta \text { ) }
$$

for any $\alpha, \beta, \gamma, \delta$ unless $\alpha=\gamma, \beta=\delta$.

Proof of Theorem 2.7. Assume that a state $\varrho$ satisfies the condition. Let $A$ be a local operator which acts on the spins in the interval $\{-L, \ldots, L\}$, and denote the restriction of $\varrho$ to $\{-L, \ldots, L\}$ by $\varrho_{L}$. Since $\varrho_{L}$ is a state on a finite system, $\varrho_{L}$ has a 
representation

$$
\varrho_{L}(A)=\sum_{a} c_{a}\left(\varphi_{a}, A \varphi_{a}\right)
$$

where $c_{a} \geqq 0, \sum_{a} c_{a}=1$, and each $\varphi_{a}$ is a normalized state which satisfies $H_{i} \varphi_{a}=0$ for $i=-L, \ldots, L-1$. By Lemma 2.8 each $\varphi_{a}$ is a linear combination of the finite volume ground states $\Omega^{(L)}{ }_{\alpha \beta}$. Writing each $\varphi_{a}$ in (2.32) as such a linear combination and using Lemmas 2.6 and 2.9, the theorem follows.

Proof of Lemma 2.8. The proof proceeds by induction in the number of sites. For two sites the most general ground state (which must have no projection onto $s=2$ ) can be written

$$
\Omega=\psi_{\alpha \beta}^{\beta \gamma} A_{\gamma}^{\alpha}
$$

where $A_{\gamma}^{\alpha}$ is an arbitrary tensor. This can be checked by noting that there are four independent states of this type, corresponding to $s=0$ and $s=1$. Now let us consider a chain of three sites with open boundary conditions. Given a ground state $\Omega$, since it has no projection onto $s=2$ for the first link, we can write it as

$$
\Omega=\psi_{\alpha \beta}^{\beta \gamma}{ }_{\delta \varepsilon} A_{\gamma}^{\alpha \delta \varepsilon}
$$

for some tensor $A_{\gamma}^{\alpha}{ }_{\gamma}^{\delta \varepsilon}$ which we may assume is symmetric in $\delta \varepsilon$ since $\psi \ldots \delta \varepsilon$ is. $\Omega$ has no projection onto $s=2$ for the second link, so we may also write

$$
\Omega=\psi_{\alpha \gamma \beta}{ }_{\beta \varepsilon} B_{\delta}^{\alpha \gamma}{ }_{\delta},
$$

for some tensor $B_{\delta}^{\alpha \gamma}{ }_{\delta}^{\varepsilon}$ which can be assumed to be symmetric in $\alpha \gamma$. Comparing these two forms of $\Omega$, we see that $A^{\alpha}{ }_{\gamma}{ }^{\delta}=0$ unless $\gamma=\delta$ or $\gamma=\varepsilon$, since otherwise $\Omega$ would contain a term in which neither index on the second site was the same as either index on the third, and this would contradict (2.34). (This term arises by choosing $\beta=\gamma$.) Since $\psi_{\alpha \beta}^{\beta \gamma}{ }_{\delta \varepsilon}$ is symmetric in $\delta \varepsilon,(2.33)$ becomes

$$
\Omega=\psi_{\alpha \beta}{ }^{\beta 1}{ }_{1 \varepsilon} A^{\alpha}{ }_{1}^{1 \varepsilon}+\psi_{\alpha \beta}{ }^{\beta 2}{ }_{2 \varepsilon} A^{\alpha}{ }_{2}{ }^{2 \varepsilon} .
$$

It only remains to prove that $A^{\alpha}{ }_{1}{ }^{1 \varepsilon}=A^{\alpha}{ }_{2}{ }^{2 \varepsilon}$. To prove this note that $B$ can also be reduced in a similar way so that

$$
\Omega=\psi_{\alpha 1}{ }^{1 \beta}{ }_{\beta \varepsilon} B^{\alpha 1}{ }_{1}{ }^{\varepsilon}+\psi_{\alpha 2}{ }_{\beta \varepsilon}{ }_{\beta \varepsilon} B_{2}^{\alpha 2}{ }_{2}{ }^{2} .
$$

Equating these two expressions we have

$$
\begin{aligned}
& \psi_{\alpha 1}{ }^{11}{ }_{1 \varepsilon}\left(A^{\alpha}{ }_{1}{ }^{1 \varepsilon}-B^{\alpha 1}{ }_{1}{ }^{\varepsilon}\right)+\psi_{\alpha 2}{ }^{22}{ }_{2 \varepsilon}\left(A^{\alpha}{ }_{2}{ }^{2 \varepsilon}-B^{\alpha 2}{ }_{2}{ }^{\varepsilon}\right) \\
& +\psi_{\alpha 1}{ }^{12}{ }_{2 \varepsilon}\left(A^{\alpha}{ }_{2}{ }^{2 \varepsilon}-B^{\alpha 1}{ }_{1}{ }^{\varepsilon}\right)+\psi_{\alpha 2}{ }^{21}{ }_{1 \varepsilon}\left(A^{\alpha}{ }_{1}{ }^{1 \varepsilon}-B^{\alpha 2}{ }_{2}{ }^{\varepsilon}\right)=0 .
\end{aligned}
$$

The coefficients of the first two terms must be zero, hence

$$
A_{1}^{\alpha}{ }_{1}^{1 \varepsilon}=B_{1}^{\alpha 1}{ }_{1}^{\varepsilon}, \quad A_{2}^{\alpha}{ }_{2}^{2 \varepsilon}=B_{2}^{\alpha 2}{ }_{2}^{\varepsilon} .
$$

The coefficient of the third term must be zero unless $\alpha=2$ and $\varepsilon=1$ in which case a contribution also arises from the fourth term,

$$
A_{2}^{\alpha}{ }^{2 \varepsilon}=B_{1}^{\alpha 1}{ }_{1}^{\varepsilon} \quad(\text { for } \alpha \varepsilon \neq 21) \text {. }
$$


Finally, the coefficient of the fourth term must be zero unless $\alpha=1$ and $\varepsilon=2$.

$$
\left.A^{\alpha}{ }_{1}^{1 \varepsilon}=B^{\alpha 2}{ }_{2}{ }^{\varepsilon} \quad \text { (for } \alpha \varepsilon \neq 12\right) .
$$

Equations (2.36), (2.37), and (2.38) imply that

$$
\left.A^{\alpha}{ }_{1}^{1 \varepsilon}=A^{\alpha}{ }_{2}{ }^{2 \varepsilon} \quad \text { (for all } \alpha, \varepsilon\right) .
$$

Thus we may write the most general ground state wave-function for three sites as

$$
\Omega=\psi_{\alpha \beta}^{\beta \gamma}{ }_{\gamma \varepsilon} C^{\alpha \varepsilon}
$$

for some tensor $C^{\alpha \varepsilon}$.

To carry out the induction step define the following states on $N+1$ sites:

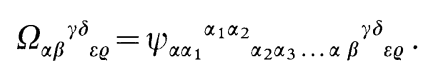

Let $\Omega$ be a ground state for $N+1$ sites. Then it is a ground state for $N$ sites and so by induction

$$
\Omega=\Omega_{\alpha \beta}^{\beta \delta}{ }_{\varepsilon \varrho} A_{\delta}^{\alpha \varepsilon} .
$$

Since $\Omega$ has no projection onto spin 2 for sites $N$ and $N+1$,

$$
\Omega=\Omega_{\alpha \beta}{ }^{\nu \delta}{ }_{\delta \varrho} B^{\alpha \beta}{ }_{\gamma}{ } .
$$

The argument for three sites can now be used to conclude

$$
\Omega=\Omega_{\alpha \beta}{ }^{\beta \delta}{ }_{\delta \varrho} C^{\alpha \varrho} .
$$

Proof of Lemma 2.9. The technique used to prove Lemma 2.6, namely Eq. (2.29), can also be used to prove Lemma 2.9. The details are left to the reader.

Next we show that any truncated correlation function for the ground state has exponential decay.

Theorem 2.10. Let $A$ and $B$ be local observables and let $d$ be the distance between their supports. Then

$$
|\omega(A B)-\omega(A) \omega(B)| \leqq 3^{-(d-2)}\|A\|\|B\| .
$$

Proof. For this proof we also write out any summations explicitly. Assume that the support of $A$ is to the left of the support of $B$ and let $r$ be the right-most site in the support of $A$ and $r+d$ the left-most site in the support of $B$. The argument is similar to that for Lemma 2.6. Choose $L$ large enough that the supports of $A$ and $B$ are in $\{-L, \ldots, L\}$. Write $\Omega^{(L)}{ }_{\alpha \beta}$ as

$$
\Omega_{\alpha \beta}^{(L)}=\sum_{\gamma, \delta} \bar{\Omega}_{\alpha}{ }^{\gamma} \Omega^{\prime}{ }_{\gamma \delta} \widetilde{\Omega}^{\delta},
$$

where $\bar{\Omega}_{\alpha}{ }^{\gamma}, \Omega^{\prime}{ }_{\gamma \delta}$, and $\widetilde{\Omega}^{\delta}{ }_{\beta}$ are now ground states on $\{-L, \ldots, r\},\{r+1, \ldots, r+d-1\}$, and $\{r+d, \ldots, L\}$, respectively.

By Lemma 2.6 we can compute $\omega(A B)$ by computing

$$
\sum_{\alpha, \beta}\left({\Omega^{(L)}}_{\alpha \beta}, A B \Omega_{\alpha \beta}^{(L)}\right)=\sum_{\alpha, \beta, \gamma, \delta, \varepsilon, \varrho}\left(\bar{\Omega}_{\alpha}^{\gamma}, A \bar{\Omega}_{\alpha}^{\varepsilon}\right)\left(\Omega_{\gamma \delta}^{\prime}, \Omega^{\prime}{ }_{\varepsilon \varrho}\right)\left(\widetilde{\Omega}_{\beta}^{\delta}, B \widetilde{\Omega}_{\beta}^{\varrho}\right) .
$$


Using (2.14) to compute $\left(\Omega^{\prime}{ }_{\gamma \delta}, \Omega^{\prime}{ }_{\varepsilon \varrho}\right)$, we see this equals

$$
\begin{aligned}
= & \left(3^{d-1}-1\right) / 2 \sum_{\alpha, \gamma}\left(\bar{\Omega}_{\alpha}^{\gamma}, A \bar{\Omega}_{\alpha}^{\gamma}\right) \sum_{\beta, \delta}\left(\widetilde{\Omega}_{\beta}^{\delta}, B \widetilde{\Omega}^{\delta}{ }_{\beta}\right) \\
& +\sum_{\alpha, \beta, \gamma, \varepsilon}\left(\bar{\Omega}_{\alpha}^{\gamma}, A \bar{\Omega}_{\alpha}^{\varepsilon}\right)\left(\widetilde{\Omega}_{\beta}^{\varepsilon}, B \widetilde{\Omega}^{\gamma}{ }_{\beta}\right) .
\end{aligned}
$$

Using Lemma 2.6 again, the first term when normalized becomes $\omega(A) \omega(B)\left(1-3^{-(d-1)}\right)$. The second term when normalized is easily bounded by a constant times $\|A\|\|B\| 3^{-(d-1)}$. Keeping track of the constants yields the result stated in the theorem.

Finally, we show that the infinite volume system has a gap. There are various definitions of the existence of a gap for the infinite volume system. We use the following one.

Definition 2.11. If $\omega$ is a ground state then $\omega$ has a gap $\varepsilon$ if for every local observable $A$ such that $\omega(A)=0$ we have

$$
\omega\left(A^{*}[H, A]\right) \geqq \varepsilon \omega\left(A^{*} A\right) .
$$

Theorem 2.12. The state $\omega$ defined in Lemma 2.6 has a gap.

Proof. Let $A$ be a local observable such that $\omega(A)=0$. Pick some $\alpha$ and $\beta$. By Lemma 2.6

$$
\lim _{L \rightarrow \infty} \frac{\left(\Omega^{(L)}{ }_{\alpha \beta}, A \Omega^{(L)}{ }_{\alpha \beta}\right)}{\left(\Omega_{\alpha \beta}^{(L)}, \Omega^{(L)}{ }_{\alpha \beta}\right)}=\omega(A)=0 .
$$

Thus the overlap of $A \Omega^{(L)}{ }_{\alpha \beta}$ with $\Omega^{(L)}{ }_{\alpha \beta}$ is $O(1)$ with respect to the norms involved as $L \rightarrow \infty$. By Lemma 2.9, the overlap of $A \Omega^{(L)}{ }_{\alpha \beta}$ with $\Omega^{(L)}{ }_{\gamma \delta}$ is $o(1)$ with respect to the norms involved unless $\alpha=\gamma$ and $\beta=\delta$. Thus the norm of the projection of $A \Omega^{(L)}{ }_{\alpha \beta}$ onto the subspace of ground states of the finite chain is $o(1)$ with respect to $\left\|A \Omega^{(L)}{ }_{\alpha \beta}\right\|$. By Theorem 2.1 the finite chain has a gap $\varepsilon$, so

$$
\left(\Omega_{\alpha \beta}^{(L)}, A^{*}[H, A] \Omega_{\alpha \beta}^{(L)}\right) \geqq(\varepsilon-O(1))\left(\Omega_{\alpha \beta}^{(L)}, A^{*} A \Omega_{\alpha \beta}^{(L)}\right) .
$$

The theorem follows.

\section{The Spin 3/2 Model on the Hexagonal Lattice}

\subsection{The Ground State}

Here we describe our results on the Valence-Bond solid state on the twodimensional hexagonal lattice. We show that the state, which is an exact ground state of a certain Hamiltonian, exhibits properties similar to those of the onedimensional VBS state. We also present models in higher dimensions which have similar ground states. Though our rigorous results are less complete than those for the one-dimensional model, they provide the first concrete examples in two and higher dimensions of exact disordered ground states which appear to be unique.

In the present subsection, we describe the model and its exact ground state precisely. Let $\Lambda$ be the set of sites in the finite hexagonal lattice with periodic boundary conditions. The set of bonds $\mathbb{B}$ consists of unordered pairs $(i, j)$ of 

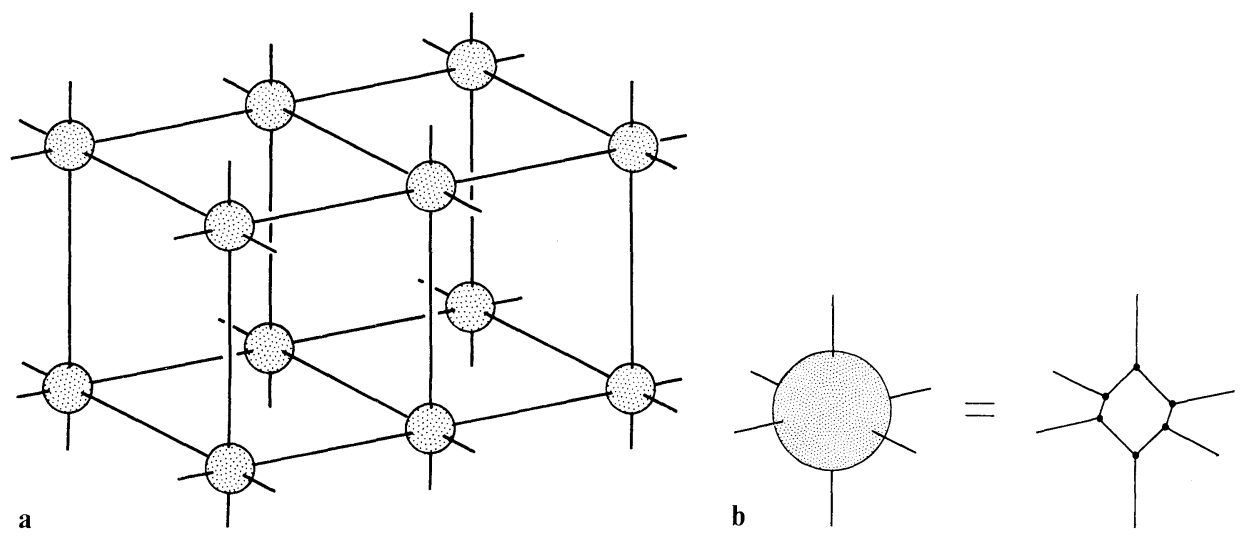

Fig. 3.1. a The cubic lattice with $\mathbf{b}$ decorated vertices is a three-dimensional bipartite lattice with coordination number three

adjacent sites $i, j$ in $\Lambda$. Note that the hexagonal lattice is a bipartite lattice, i.e., it can be decomposed into two sublattices $\Lambda_{A}$ and $\Lambda_{B}$ with the properties that $\Lambda_{A} \cup \Lambda_{B}=\Lambda, \Lambda_{A} \cap \Lambda_{B}=\emptyset$, and for any $(i, j) \in \mathbb{B}$, either $i \in \Lambda_{A}, j \in \Lambda_{B}$ or $j \in \Lambda_{A}, i \in \Lambda_{B}$.

All the results in the present section can be generalized to arbitrary bipartite lattices with coordination number three. Among the examples of such lattices are the one-dimensional ladder lattice, the two-dimensional hexagonal lattice, and various lattices [43] with dimensions three or higher. An example of such a lattice in three dimensions is given in Fig. 3.1. We can modify this lattice so that all the bonds have unit length, and any pair of sites separated by unit length are connected by a bond. However, we note that such an example is primarily of theoretical interest and has little direct connection with physical experiments. The importance of these examples is that they show that isotropic antiferromagnets in dimensions greater than one may exhibit the same properties that Haldane predicted for the Heisenberg chain. (In Sect. 4, we discuss lattices with coordination numbers greater than three.)

Let us associate a spin operator $\mathbf{S}_{i}$ with spin $s=3 / 2$ with site $i$. Then our Hamiltonian is

$$
H=\sum_{(i, j) \in \mathbb{B}} P_{3}\left(\mathbf{S}_{i}+\mathbf{S}_{j}\right)=\sum_{(i, j) \in \mathbb{B}} H_{i j},
$$

where $P_{3}(\mathbf{S})$ is the orthogonal projection onto the states with $\mathbf{S}^{2}=3(3+1)$ (spin 3$)$.

Note that our Hamiltonian describes antiferromagnetic pairwise interactions with perfect $S O(3)$ symmetry. To see that (3.1) has an antiferromagnetic character, observe that the energy is higher when the two neighboring spins add up to the maximum spin. This Hamiltonian is also equivalent to the following polynomial of the spin operators.

$$
\sum_{(i, j) \in \mathbb{B}}\left[\mathbf{S}_{i} \cdot \mathbf{S}_{j}+\frac{116}{243}\left(\mathbf{S}_{i} \cdot \mathbf{S}_{j}\right)^{2}+\frac{16}{243}\left(\mathbf{S}_{i} \cdot \mathbf{S}_{j}\right)^{3}\right] .
$$

To extend the VBS ground state to the hexagonal lattice, we think of spin $3 / 2$ as the symmetric part of the product of three spin 1/2's. For a single site, our basis 
consists of four vectors $\psi_{111}, \psi_{112}=\psi_{121}=\psi_{211}, \psi_{122}=\psi_{212}=\psi_{221}$, and $\psi_{222}$ which are defined as

$$
\psi_{\alpha_{1} \alpha_{2} \alpha_{3}}=\sum_{\left(\alpha_{1}, \alpha_{2}, \alpha_{3}\right) \rightarrow\left(\beta_{1}, \beta_{2}, \beta_{3}\right)} \psi_{\beta_{1}} \otimes \psi_{\beta_{2}} \otimes \psi_{\beta_{3}} / \sqrt{3 !} .
$$

Here $\left\{\psi_{1}=(+), \psi_{2}=(-)\right\}$ denotes the standard $S^{3}$ eigenstate basis for spin $1 / 2$, and the summation runs over all the permutations of $\left(\alpha_{1}, \alpha_{2}, \alpha_{3}\right)$. The normalization condition for these vectors is

$$
\left(\psi_{\alpha_{1} \alpha_{2} \alpha_{3}}, \psi_{\beta_{1} \beta_{2} \beta_{3}}\right)=\sum_{\left(\beta_{1}, \beta_{2}, \beta_{3}\right) \rightarrow\left(\gamma_{1}, \gamma_{2}, \gamma_{3}\right)} \delta_{\alpha_{1} \gamma_{1}} \delta_{\alpha_{2} \gamma_{2}} \delta_{\alpha_{3} \gamma_{3}} .
$$

Therefore, our basis is related to the standard $S^{3}$ eigenstate basis for $s=3 / 2$ by the following.

$$
\begin{array}{ll}
(+3 / 2)=\psi_{111} / \sqrt{6}, & (+1 / 2)=\psi_{112} / \sqrt{2}, \\
(-1 / 2)=\psi_{122} / \sqrt{2}, & (-3 / 2)=\psi_{222} / \sqrt{6} .
\end{array}
$$

Again we use the following convention for raising indices

$$
\psi^{\alpha_{1} \alpha_{2} \alpha_{3}}=\varepsilon^{\alpha_{1} \beta_{1}} \varepsilon^{\alpha_{2} \beta_{2}} \varepsilon^{\alpha_{3} \beta_{3}} \psi_{\beta_{1} \beta_{2} \beta_{3}}
$$

and a summation convention for repeated upper and lower indices.

Let $i_{1}, i_{2}$, and $i_{3}$ be the three neighboring sites of a site $i$. We represent a state on site $i$ as

$$
\psi_{\alpha_{i i_{1}} \alpha_{i 2} \alpha_{2 l_{3}}}
$$

if $i \in \Lambda_{A}$, and as

$$
\psi^{\alpha_{i i_{1}} \alpha_{2 l_{2}} \alpha_{i i_{3}}}
$$

if $i \in \Lambda_{B}$. Then the VBS state on the hexagonal lattice is obtained by letting each pair of spin $1 / 2$ 's on adjacent sites form a singlet pair.

$$
\Omega_{\Lambda}=\bigotimes_{i \in \Lambda_{A}} \psi_{\alpha_{l l_{1} \alpha_{l l_{2}} \alpha_{l l_{3}}}} \bigotimes_{j \in \Lambda_{B}} \psi^{\alpha_{J J_{1}} \alpha_{J_{2}} \alpha_{J_{3}}} .
$$

Note that for any $(i, j) \in \mathbb{B}$, both indices $\alpha_{i j}$ and $\alpha_{j i}$ appear exactly once in the above expression. Since one of them appears as an upper index, and the other appears as a lower index, we can sum over them by setting $\alpha_{i j}=\alpha_{j i}$. Then all the indices in (3.4) are contracted, and we get a single state $\Omega_{\Lambda}$ where the valence bond structure is identical to the bond structure of the lattice (Fig. 3.2).

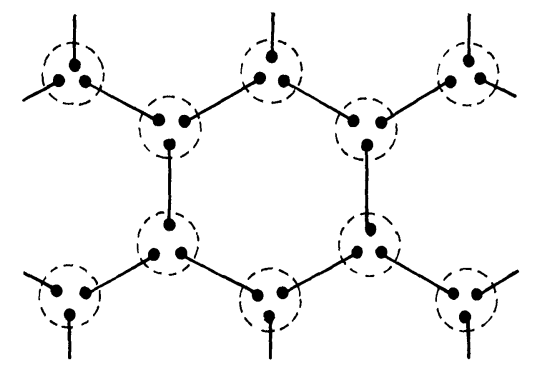

Fig. 3.2. The VBS state on the hexagonal lattice. Each dot, line, and dotted circle represents a spin $1 / 2$, a singlet pair, and the symmetrization of three spin $1 / 2$ 's to create a spin $3 / 2$ 
To see that the state $\Omega_{\Lambda}$ is actually an exact ground state of the Hamiltonian (3.1), note that the restriction of the state to an arbitrary pair of adjacent sites contains at least one singlet pair. Therefore, the total spin of these two sites is always 2, 1 or 0 , which means $H_{i j} \Omega_{A}=0$ for any $(i, j) \in \mathbb{B}$. Since 0 is the minimum eigenvalue of the local Hamiltonian $H_{i j}, \Omega_{A}$ is an exact ground state.

It is desirable to have an argument for the uniqueness of the infinite volume ground state (like Theorem 2.7) for the hexagonal lattice model (3.1). In fact, we expect the analog of Lemma 2.8 (finite volume lemma) to be valid for the general class of VBS systems (which is described in Sects. 4 and 6) including the present one. Moreover, our result on the exponential decay of the two point correlation function suggests that the analog of Lemma 2.6 is also valid in the present model (for a bipartite lattice with coordination number three). See note added in proof.

\subsection{Properties of the Ground State}

The main result of the present section is in the following theorem which establishes the exponential decay of the correlation function in the VBS state (3.4). The proof of the theorem will be presented in the next subsection.

Theorem 3.1. The two point correlation function of the VBS state with periodic boundary conditions (3.4)

$$
\left\langle\mathbf{S}_{i} \cdot \mathbf{S}_{j}\right\rangle_{\Lambda}=\frac{\left(\Omega_{\Lambda}, \mathbf{S}_{i} \cdot \mathbf{S}_{j} \Omega_{\Lambda}\right)}{\left(\Omega_{\Lambda}, \Omega_{\Lambda}\right)}
$$

satisfies the bound

$$
0 \leqq(-1)^{i-j}\left\langle\mathbf{S}_{i} \cdot \mathbf{S}_{j}\right\rangle_{\Lambda} \leqq C \exp \left(-|i-j| / \xi_{0}\right)
$$

for any $i, j \in \Lambda$. Here $C$ and $\xi_{0}$ are positive constants which are independent of the lattice size, $(-1)^{i-j}=1$ or -1 according to whether $i$ and $j$ belong to the same sublattice or not, and $|i-j|$ denotes the graph theoretic distance (i.e., the minimum number of bonds needed to connect $i$ and $j$.)

Note that $\xi_{0}$ in the theorem provides an upper bound for the actual correlation length. From our proof in the next subsection, we get $\xi_{0}=\{\ln (2 / \sqrt{6})\}^{-1}=4.93 \ldots$. For the hexagonal lattice, we may use the "exact but nonrigorous" value [33] of the entropy of self-avoiding random walks to get an improved upper bound $\xi_{1}=3.54 \ldots$.

Let us describe some physical consequences of our theorem. Consider the following infinite volume limit of the VBS state $\Omega_{A}$,

$$
\omega(A)=\lim _{\Lambda \rightarrow \Lambda_{\infty}} \frac{\left(\Omega_{\Lambda}, A \Omega_{\Lambda}\right)}{\left(\Omega_{\Lambda}, \Omega_{\Lambda}\right)} .
$$

Here $\Lambda_{\infty}$ denotes the infinite hexagonal lattice. By symmetry

$$
\omega\left(\mathbf{S}_{i}\right)=\lim _{\Lambda \rightarrow \Lambda_{\infty}}\left\langle\mathbf{S}_{i}\right\rangle_{\Lambda}=(0,0,0) .
$$


At the same time, since our upper bound (3.6) is uniform in $\Lambda$, the corresponding bound is also valid for the correlation function $\omega\left(\mathbf{S}_{i} \cdot \mathbf{S}_{j}\right)$ of infinite volume VBS state (3.7). This fact strongly suggests that $\omega$ is a pure state.

Therefore, we conjecture that $\omega$ is the unique infinite volume ground state of the Hamiltonian (3.1), and has no Néel ordering. It has been argued that ground states of two-dimensional antiferromagnets usually have Néel order. Though some examples of non Néel ordered exact ground states are known [27], they are infinitely degenerate and exhibit trivial ultra short range correlation functions (see also Sect. 6). As far as we know, the VBS states (3.4) are the first examples in two and higher dimensions of disordered exact ground state which are presumably unique. Note that the VBS state is qualitatively different from Anderson's disordered ground state (resonating valence-bond) on the triangular lattice [7]. However, it is interesting that valence bonds seem to play crucial roles in both cases.

The exponential fall-off of the correlation function also suggests the existence of an energy gap in the Hamiltonian (3.1). However, we do not know any way of proving (or disproving) this conjecture.

Finally, let us briefly discuss the relevance of our results and speculations to Hamiltonians other than (3.1). First of all the (conjectured) uniqueness of the ground state and the (conjectured) existence of an energy gap suggest that our hexagonal lattice VBS state is in some sense stable under a perturbation of the Hamiltonian. In particular, if we start from the Hamiltonian (3.1) and add some "small" perturbation, the resulting ground state is expected to have the same properties as the VBS state, i.e., exponentially decaying correlation functions, an energy gap, and no Néel order. We say that such a ground state is in the VBS phase. Then a natural question is whether a system with the most standard (and realistic) purely bilinear Heisenberg Hamiltonian

$$
H=\sum_{(i, j) \in \mathbb{B}} \mathbf{S}_{i} \cdot \mathbf{S}_{j}
$$

is in the VBS phase or not. As we discuss in the appendix, the reflection positivity argument of $[21,26]$ is strong enough to prove the existence of Néel order in the ground state of an $s=3 / 2$ antiferromagnet on the hexagonal lattice with bilinear Hamiltonian (3.8). Thus as we change the Hamiltonian continuously from $\left(3.1^{\prime}\right)$ to (3.8), a (second order?) phase transition from the VBS phase to the Néel ordered phase takes place at a certain Hamiltonian. Moreover, it is also possible that the VBS phase is realized in Hamiltonians which are not at all close to (3.1). For example, a bilinear Hamiltonian with nonnearest neighbor couplings sufficiently large to destroy Néel order could be in the VBS phase.

\subsection{Proof of Exponential Decay}

The present subsection is devoted to the proof of Theorem 3.1. The starting point of our proof is the following polymer representation of the VBS state (3.4). Note that in (3.4), there is a one-to-one correspondence between the lattice bonds and the pairs of repeated upper and lower indices. Therefore, each term in (3.4) can be represented by assigning the values $\alpha_{i j}=1$ or 2 to every bond in the lattice. By 
denoting the set of bonds with $\alpha_{i j}=1$ by $G$, we can rewrite (3.4) as

$$
\Omega_{\Lambda}=\sum_{G \subset \mathbb{B}} \Phi\left(\left\{n_{k}(G)\right\}_{k \in \Lambda}\right),
$$

where $G$ runs over all the subsets of the lattice bonds. $n_{k}(G)$ denotes the number of the bonds in $G$ incident to a site $k$, and the basis states are given by

$$
\Phi\left(\left\{n_{k}(G)\right\}_{k \in \Lambda}\right)=\bigotimes_{l \in \Lambda_{A}} \psi_{n_{l}(G)} \bigotimes_{m \in \Lambda_{B}} \psi^{n_{m}(G)} .
$$

Here $\psi_{n}$ (respectively, $\left.\psi^{n}\right)(n=0,1,2)$ denotes the basis vector $\psi_{\alpha_{1} \alpha_{2} \alpha_{3}}$ (respectively, $\left.\psi^{\alpha_{1} \alpha_{2} \alpha_{3}}\right)$, where $n$ of the three indices are 1 .

Remark. The polymer representation (3.9) is also valid for the $s=1$ VBS state on the one dimensional chain where our basis is related to the standard $S^{3}$ eigenstate basis as

$$
\psi_{0}=\psi^{2}=\sqrt{2}(+), \quad \psi_{1}=-\psi^{1}=(0), \quad \psi_{2}=\psi^{0}=\sqrt{2}(-) .
$$

Inserting this relation into (3.9) and rewriting each term in the standard basis, we get the representation of the $s=1$ VBS state presented at the end of Sect. 2.1.

Consider the norm $\left(\Omega_{\Lambda}, \Omega_{\Lambda}\right)$ of the VBS state (3.4). From (3.9) and the inner product formula (3.3), we easily find that

$$
\begin{aligned}
\left(\Omega_{\Lambda}, \Omega_{\Lambda}\right) & =\sum_{G^{\prime}, G \subset \mathbb{B}}\left(\Phi\left(\left\{n_{k}\left(G^{\prime}\right)\right\}_{k \in \Lambda}\right), \Phi\left(\left\{n_{k}(G)\right\}_{k \in \Lambda}\right)\right) \\
& =\sum_{G^{\prime}, G \subset \mathbb{B}} \prod_{k \in \Lambda} \chi\left(n_{k}\left(G^{\prime}\right)=n_{k}(G)\right) n_{k}(G) !\left(3-n_{k}(G)\right) !,
\end{aligned}
$$

where the characteristic function $\chi(A)$ is 1 if $A$ is true, and 0 if $A$ is false. It is useful to represent the above expression geometrically. For a given pair $G^{\prime}, G$, we draw a graph like Fig. 3.3a by putting a straight segment on each bond in $G$, and a wavy segment on each bond in $G^{\prime}$. From (3.11), we find that a graph contributes to the norm $\left(\Omega_{\Lambda}, \Omega_{\Lambda}\right)$ if and only if for each site the number of straight segments incident to the site equals the number of wavy segments incident to the site.

Let $S^{+}=\left(S^{1}+i S^{2}\right) / 2$ and $S^{-}=\left(S^{1}-i S^{2}\right) / 2$ be the usual raising and lowering operators, respectively. It is easy to check that these operators acting on our basis give

$$
S^{+} \psi_{n}=(3-n) \psi_{n+1}, \quad S^{-} \psi_{n}=n \psi_{n-1} .
$$
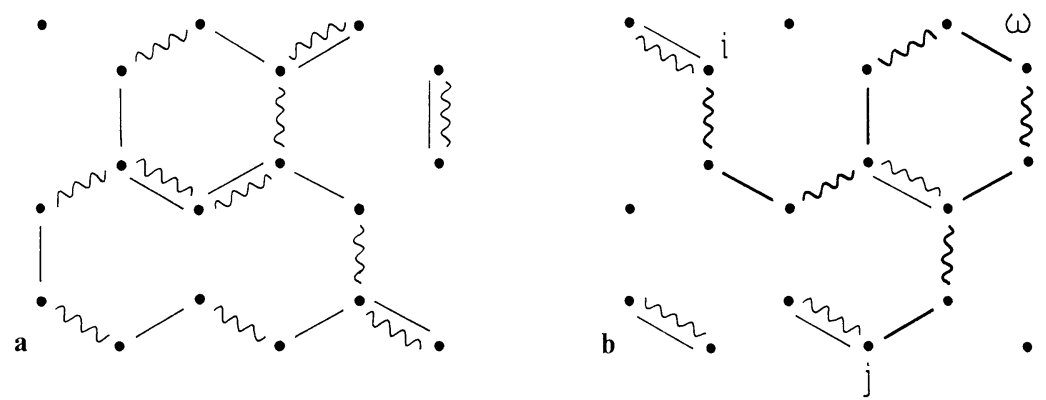

Fig. 3.3. a A graph which contributes to $\left(\Omega_{A}, \Omega_{A}\right)$. b A graph which contributes to $\left(\Omega_{A}, S^{+}{ }_{i} S^{-}{ }_{j} \Omega_{A}\right)$. The thicker lines represent the self-avoiding walk $\omega$ 
Let $i$ and $j$ be sites in $\Lambda_{B}$. Then from (3.10) and (3.12), we find

$$
S_{i}^{+} S^{-}{ }_{j} \Phi\left(\left\{n_{k}(G)\right\}_{k \in \Lambda}\right)=\left(3-n_{i}(G)\right) n_{j}(G) \Phi\left(\left\{n_{k}(G)+\delta_{k, i}-\delta_{k, j}\right\}_{k \in \Lambda}\right) .
$$

This formula and (3.9) imply the following expression for the unnormalized correlation function:

$$
\begin{aligned}
& \left(\Omega_{\Lambda}, S^{+}{ }_{i} S^{-}{ }_{j} \Omega_{\Lambda}\right) \\
& \quad=\sum_{G^{\prime}, G \subset \mathbb{B}}\left(3-n_{i}(G)\right) n_{j}(G)\left(\Phi\left(\left\{n_{k}\left(G^{\prime}\right)\right\}_{k \in \Lambda}\right), \Phi\left(\left\{n_{k}(G)+\delta_{k, i}-\delta_{k, j}\right\}_{k \in \Lambda}\right)\right) \\
& \quad=\sum_{G^{\prime}, G} \prod_{k \in \Lambda} \chi\left(n_{k}\left(G^{\prime}\right)=n_{k}(G)+\delta_{k, i}-\delta_{k, j}\right)\left(n_{k}(G)+\delta_{k, i}\right) !\left(3-n_{k}(G)+\delta_{k, j}\right) ! .
\end{aligned}
$$

Again we represent the above formula geometrically. This time, the same number of straight and wavy segments must be incident to each site in $\Lambda$ except $i$ and $j$. At site $i$ we must have one extra wavy segment, and at site $j$ we must have one extra straight segment (Fig. 3.3b). This observation allows us to construct a selfavoiding random walk connecting $i$ and $j$ as follows.

Consider an arbitrary pair of $G^{\prime}, G$ which contributes to (3.13), and the corresponding graph of straight and wavy segments. Then from the above property we can find at least one bond $\left(i, k_{1}\right)$ which is occupied only by a wavy segment (Fig. 3.3b). [If there exist more than one (i.e., two) such bonds, we choose one of them by a suitable convention.] If $k_{1} \neq j$, there are equal numbers of straight and wavy segments incident to $k_{1}$. Since the bond $\left(k_{1}, i\right)$ is occupied only by a wavy segment, this implies that there exists a unique bond $\left(k_{1}, k_{2}\right)$ occupied only by a straight segment. By repeating the same procedure, we get a sequence of bonds $\left(i, k_{1}\right),\left(k_{1}, k_{2}\right),\left(k_{2}, k_{3}\right), \ldots$ occupied only by straight or wavy segments in an alternating way (Fig. 3.3b). Clearly, this sequence terminates only when we hit the site $j$, and thus it forms a self-avoiding walk $\omega=\left\{k_{0}, k_{1}, \ldots, k_{n}\right\}$, where $k_{0}=i, k_{n}=j$, $\left(k_{i}, k_{i+1}\right) \in \mathbb{B}$ for any $i$, and $k_{l} \neq k_{m}$ if $l \neq m$. We stress that such a self-avoiding walk is always determined uniquely from any pair $G^{\prime}, G$ which contributes to (3.13), if we fix a convention for the choice of the first step of the walk at site $i$. Let us denote this unique self-avoiding walk by $\omega\left(G^{\prime}, G\right)$.

Now from (3.11), (3.13), and the above construction, we can write down the following random walk representation for the two point correlation function:

$$
\left\langle S^{+}{ }_{i} S^{-}{ }_{j}\right\rangle=\frac{\left(\Omega_{\Lambda}, S^{+}{ }_{i} S^{-}{ }_{j} \Omega_{\Lambda}\right)}{\left(\Omega_{\Lambda}, \Omega_{\Lambda}\right)}=\sum_{\omega: i \rightarrow j} W(\omega) .
$$

Here $\omega$ runs over all the self-avoiding walks on the lattice connecting $i$ and $j$, and the statistical weight $W(\omega)$ is defined by

$$
=\frac{\sum_{G^{\prime}, G \subset \mathbb{B}} \chi\left(\omega\left(G^{\prime}, G\right)=\omega\right) \prod_{k \in \Lambda} \chi\left(n_{k}\left(G^{\prime}\right)=n_{k}(G)+\delta_{k, i}-\delta_{k, j}\right)\left(n_{k}(G)+\delta_{k, i}\right) !\left(3-n_{k}(G)+\delta_{k, j}\right) !}{\sum_{G^{\prime}, G \subset \mathbb{B}} \prod_{k \in \Lambda} \chi\left(n_{k}\left(G^{\prime}\right)=n_{k}(G)\right) n_{k}(G) !\left(3-n_{k}(G)\right) !} .
$$

When $i$ and $j$ are not both in $\Lambda_{B}$, we can also carry out the same construction and get similar random walk representations. Here the weight $W(\omega)$ has an overall factor $(-1)^{i-j}$ which comes from the analog of (3.12) for sites in $\Lambda_{A}$. 
In the following we will show that for any $\omega$ this statistical weight can be bounded as

$$
W(\omega) \leqq C_{1}(\sqrt{6})^{-|\omega|},
$$

where $|\omega|$ denotes the length of the walk $\omega$ (i.e., the number of bonds in $\omega$ ), and $C_{1}$ is a finite constant independent of the lattice size.

Combining (3.14) and (3.16) we get

$$
\left\langle S^{+}{ }_{i} S^{-}{ }_{j}\right\rangle \leqq C_{1} \sum_{\omega: i \rightarrow j}(\sqrt{6})^{-|\omega|} \leqq C_{1} \sum_{l=|i-j|}^{\infty} N(l)(\sqrt{6})^{-l},
$$

where $N(l)$ denotes the number of distinct self-avoiding walks with length $l$. Since the coordination number of the lattice is three, $N(l)$ can be bounded by its mean field behavior as $N(l) \leqq 2^{l}$. Inserting this bound in the above inequality and summing over $l$, we get

$$
\left\langle S^{+}{ }_{i} S^{-}{ }_{j}\right\rangle \leqq C_{2} \exp \left(-|i-j| / \xi_{0}\right)
$$

where $\xi_{0}=\{\ln (2 / \sqrt{6})\}^{-1}=4.93 \ldots$, and $0<C_{2}<\infty$ is independent of the lattice size. Since $\left\langle S^{+}{ }_{i} S^{-}{ }_{j}\right\rangle=\left(\left\langle S^{1}{ }_{i} S^{1}{ }_{j}\right\rangle+\left\langle S^{2}{ }_{i} S^{2}{ }_{j}\right\rangle\right) / 4$, the $S O(3)$ symmetry of the system implies the desired bound (3.6) for the correlation function $\left\langle\mathbf{S}_{i} \cdot \mathbf{S}_{j}\right\rangle$. For the hexagonal lattice, the exact (but nonrigorous) behavior of $N(l)$ is known to be $N(l)$ $=(\sqrt{2+\sqrt{2}})^{l+o(l)}[33]$. Using this instead of the above crude mean field upper bound, we get an improved upper bound $\xi_{1}=3.54 \ldots$ for the correlation length.

Now let us turn to the proof of the bound (3.16) for the statistical weight of the random walks. Fix a random walk $\omega$. Denote the numerator and denominator of (3.15) by $Z_{\omega}$ and $Z$, respectively. We will construct a lower bound for $Z$ in terms of $Z_{\omega}$. Consider a graph for a pair $G^{\prime}, G$ which contributes to $Z_{\omega}$. If we eliminate all the segments in the graph which are on the walk $\omega$, we will get a graph in which the same numbers of straight and wavy segments are incident to any site. Moreover, this property is preserved if we put both a straight and a wavy segment on any bond in the trajectory of the eliminated walk $\omega$. In this way, we get $2^{|\omega|}$ distinct graphs which contribute to the norm $Z$ (Fig. 3.4a-c). Note that given any one of these $2^{|\omega|}$ graphs we can recover $G^{\prime}, G$. (Recall that $\omega$ is fixed.) Thus each term in $Z$ appears at most once as one of these $2^{|\omega|}$ graphs for some $G^{\prime}, G$.
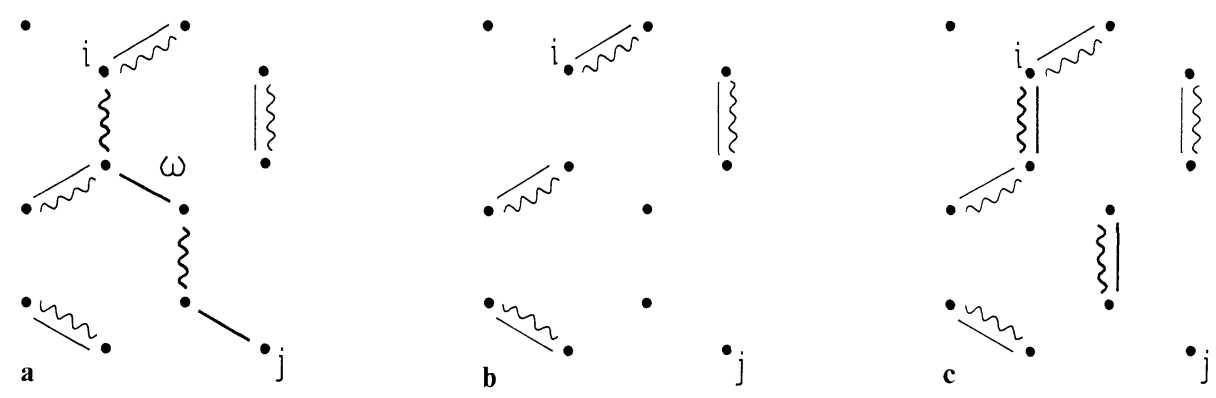

Fig. 3.4. a A typical term in $Z_{\omega}$. b The result of deleting the lines in $\omega$. $\mathbf{c}$ One of the $2^{|\omega|}$ terms in $Z$ which are associated with $\mathbf{a}$ 
We have shown that with each term $G^{\prime}, G$ in $Z_{\omega}$ we may associate $2^{|\omega|}$ terms in $Z$. These $2^{|\omega|}$ terms need not have the same weight as the original term in $Z_{\omega}$. To compare the weights, first note that the local weights $n_{k}(G) !\left(3-n_{k}(G)\right)$ ! and $\left(n_{k}(G)+\delta_{k, i}\right) !\left(3-n_{k}(G)+\delta_{k, j}\right) !$ only take on the values 3 ! and 2 ! for sites $k \neq i, j$. For a site $k \neq i, j$ which belongs to $\omega, n_{k}(G)$ must be 1 or 2 , so the local weight $n_{k}(G)$ ! $\left(3-n_{k}(G)\right)$ ! equals 2 !. Thus the weight appearing in $Z_{\omega}$ is always less than or equal to the weight appearing in $Z$. This yields the bound

$$
Z \geqq \text { const } 2^{|\omega|} Z_{\omega} \text {. }
$$

To obtain the stronger bound (3.16) we need to take advantage of the fact that the local weight in $Z$ is sometimes 3 !. At the sites $k_{2}, k_{4}, k_{6}, \ldots$ we simply bound the local weight in $Z$ below by 2 !. Let $k$ be one of the sites $k_{3}, k_{5}, \ldots$ other than $j$. The bond incident to $k$ which does not belong to $\omega$ must either be empty or contain both a straight and a wavy segment. Suppose it is empty. Then when both of the bonds in $\omega$ incident to $k$ are empty, the local weight at $k$ for the term in $Z$ is 3 !. If at least one of the bonds in $\omega$ incident to $k$ has both a straight and wavy segment, then the local weight is 2 !. Thus out of the four possibilities for the two bonds in $\omega$ incident to $k$, exactly three have a local weight of 2 ! and exactly one has a local weight of 3 !. If the bond incident to $k$ which is not on $\omega$ contains both a straight and a wavy segment, then we find the same conclusion is true. Hence for every two bonds in $\omega$, we obtain a factor of $3+3 \cdot 1=6$, instead of just $2 \cdot 2=4$. Inequality (3.16) follows.

\section{VBS States on an Arbitrary Lattice}

\subsection{The Ground States}

In the present section, we extend the VBS state to an arbitrary lattice, and discuss its properties.

Let $\Lambda$ be the set of sites in a translation invariant finite lattice with periodic boundary conditions and coordination number $z$. The set of bonds $\mathbb{B}$ consists of unordered pairs $(i, j)$ of adjacent sites $i, j$ in $\Lambda$. We also assume that the lattice is bipartite, i.e., it can be decomposed into two sublattices $\Lambda_{A}, \Lambda_{B}$ with the properties $\Lambda_{A} \cup \Lambda_{B}=\Lambda, \Lambda_{A} \cap \Lambda_{B}=\emptyset$ and for any $(i, j) \in \mathbb{B}$, either $i \in \Lambda_{A}, j \in \Lambda_{B}$ or $j \in \Lambda_{A}, i \in \Lambda_{B}$.

Let us associate a spin operator $\mathbf{S}_{i}$ with spin $s=z / 2$ with site $i$. Consider the $S O(3)$ symmetric antiferromagnetic Hamiltonian

$$
H=\sum_{(i, j) \in \mathbb{B}} P_{z}\left(\mathbf{S}_{i}+\mathbf{S}_{j}\right)=\sum_{(i, j) \in \mathbb{B}} H_{i j},
$$

where $P_{z}(\mathbf{S})$ is the orthogonal projection onto the states with $\mathbf{S}^{2}=z(z+1)(\operatorname{spin} z)$.

The VBS ground state is again constructed by first regarding spin $z / 2$ as the symmetric part of the product of $z$ spin 1/2's, and then letting each pair of spin 1/2's on the adjacent sites form a singlet pair. In this way, the valence bond structure again becomes identical to the bond structure $\mathbb{B}$ of the lattice.

To describe the VBS state precisely, we again make use of an $S U(2)$ notation. For a single spin with $s=z / 2$, our basis consists of the following vectors with $\alpha_{i}=1$ or 2:

$$
\psi_{\alpha_{1} \ldots \alpha_{z}}=\sum_{\left(\alpha_{1}, \ldots, \alpha_{z}\right) \rightarrow\left(\beta_{1}, \ldots, \beta_{z}\right)} \psi_{\beta_{1}} \otimes \ldots \otimes \psi_{\beta_{z}} / \sqrt{z !} .
$$


Here $\left\{\psi_{1}=(+), \psi_{2}(-)\right\}$ denotes the standard $S^{3}$ eigenstate basis for an $s=1 / 2$ spin, and the summation runs over all the permutations of $\left(\alpha_{1}, \ldots, \alpha_{z}\right)$. These states satisfy the following normalization condition:

$$
\left(\psi_{\alpha_{1} \ldots \alpha_{z}}, \psi_{\beta_{1} \ldots \beta_{z}}\right)=\sum_{\left(\beta_{1}, \ldots, \beta_{z}\right) \rightarrow\left(\gamma_{1}, \ldots, \gamma_{z}\right)} \delta_{\alpha_{1} \gamma_{1}} \delta_{\alpha_{2} \gamma_{2}} \ldots \delta_{\alpha_{z} \gamma_{z}} .
$$

Let $i_{1}, \ldots, i_{z}$ denote the $z$ neighboring sites of a site $i$. We represent a state on site $i$ as

if $i \in \Lambda_{A}$, and as

$$
\psi_{\alpha_{i i_{1} \alpha_{i i_{2}} \ldots \alpha_{i i_{z}}}}
$$

$$
\psi^{\alpha_{i i_{1}} \alpha_{i i_{2}} \ldots \alpha_{i i_{z}}}
$$

if $i \in \Lambda_{B}$. Here the state with the raised indices is defined as before. Then the VBS state on the general lattice $\Lambda$ can be expressed as

$$
\Omega_{\Lambda}=\bigotimes_{i \in \Lambda_{A}} \psi_{\alpha_{\imath i_{1}} \alpha_{i i_{2}} \ldots \alpha_{u_{z}}} \bigotimes_{j \in \Lambda_{B}} \psi^{\alpha_{j j_{1}} \alpha_{j j_{2}} \ldots \alpha_{j j_{z}}}
$$

As in Sect. 3.1, we identify $\alpha_{i j}$ with $\alpha_{j i}$, and sum over all the repeated upper and lower indices. Then all the indices in (4.4) are contracted, and we get a single state $\Omega_{\Lambda}$. Again it is quite easy to check that the VBS state (4.4) is an exact ground state of the Hamiltonian (4.1) in the sense that $H_{i j} \Omega_{\Lambda}=0$ holds for any $(i, j) \in \mathbb{B}$.

As in the hexagonal lattice VBS state, we do not have any uniqueness argument like Theorem 2.7. Though we believe that an analog of Lemma 2.8 (finite volume lemma) is true, the uniqueness in the infinite volume limit seems not to be true in general. We return to this point later.

In Sects. 2 and 3, we have seen that the VBS state on the one-dimensional chain and two-dimensional hexagonal lattice seem to share common properties, namely, the exponential decay of correlations, the absence of Néel order, and the existence of an energy gap. Thus one might well conclude that these are general features of all VBS states. However, in the following subsections we will present a Cayley tree model and a stochastic geometric argument which suggest this is not the case. As we summarize in the following conjecture, the VBS state on certain lattices may be in a completely different phase.

Conjecture. If the coordination number $z$ and the dimension $d$ are sufficiently small, the system (4.1) is in the VBS phase, i.e., it has a unique (infinite volume) ground state with exponentially decaying correlation functions, no Néel order, and a finite energy gap. However, if $z$ and $d$ are sufficiently large, the system (4.1) is in the Néel ordered phase.

As we have noted in the previous section, we expect (but cannot prove) that the VBS state does not have Néel order if the coordination number of the lattice is three. Since we can construct a lattice with coordination number three in any dimension $d$, not only large $d$ but also large $z$ is actually necessary for the existence of Néel order.

When the system is in the Néel ordered phase, the infinite volume limit of the VBS state (4.4) constructed using periodic boundary conditions cannot be a pure 
state since this VBS state has no $S O(3)$ symmetry breaking. A pure state with explicit Néel order may be obtained by the following procedure. Consider a finite lattice $\Lambda$ with boundary $\partial \Lambda$, and construct the VBS state on $\Lambda$. The only difference from the periodic boundary condition case (4.4) is that at each site in $\partial \Lambda$ several spin 1/2's remain uncontracted. It is clear that for any choice of these boundary spin $1 / 2$ 's, the VBS state is an exact ground state in the sense that $H_{i j} \Omega_{\Lambda}=0$ for any bond $(i, j)$. Consider the boundary conditions where we fix the boundary spin $1 / 2$ 's to $(+)$ for the sites in $\partial \Lambda \cap \Lambda_{A}$, and to $(-)$ for the sites in $\partial \Lambda \cap \Lambda_{B}$. By taking the infinite volume limit of the VBS states with these boundary conditions, we should get a pure ground state with nonzero staggered magnetization, provided that the system is in the Néel ordered phase. In the next subsection, we will carry out this procedure for the Cayley tree and show that there is Néel order when the coordination number exceeds four.

The existence of Néel order in valence bond states is not as surprising as it appears. Though a valence bond carries strong quantum fluctuations, it also has a tendency to make the two spins "point" in opposite directions. The behavior of the VBS (or other valence bond) states in the infinite volume limit is determined by the competition of these two effects.

\subsection{The VBS State on the Cayley Tree}

In the present subsection, we describe our results on the VBS state on the Cayley tree. Let $C_{N}$ be the $N$-th order Cayley tree with coordination number $z$ (Fig. 4.1a), and $\partial C_{N}$ be its boundary. We assume $N$ is even. Again we decompose $C_{N}$ into two sublattices $\Lambda_{A}$ and $\Lambda_{B}$, so that the boundary $\partial C_{N}$ and the origin are contained in the sublattice $\Lambda_{B}$. Then the VBS state on the Cayley tree is

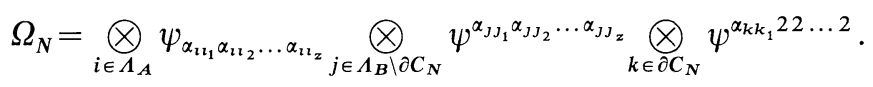

In order to test for the existence of Néel order, we have set the boundary $s=1 / 2$ spins to $(+)$.
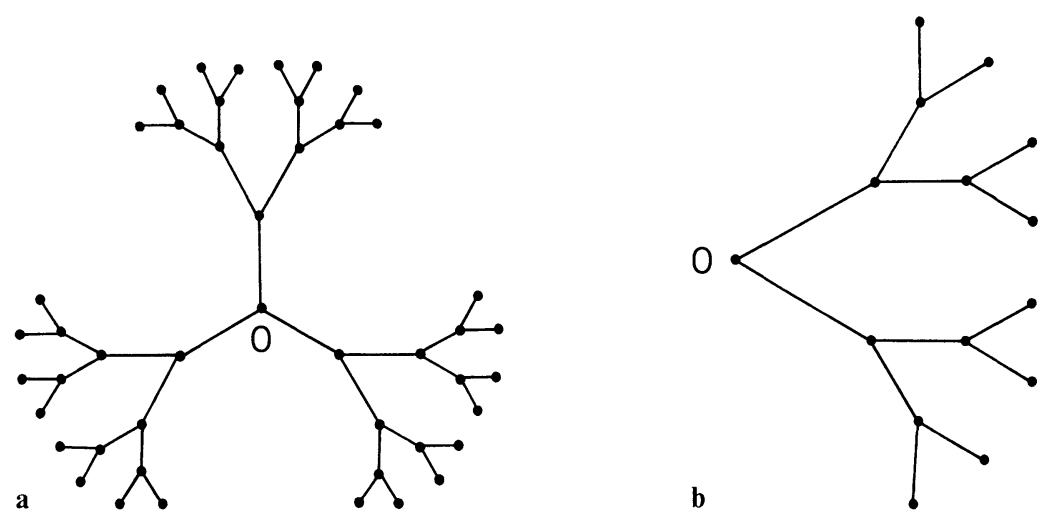

Fig. 4.1. a The fourth order Cayley tree $C_{4}$ with coordination number three, and $\mathbf{b}$ the corresponding branch $B_{4}$ 
Let us define the Néel order parameter by

$$
m=\lim _{N \rightarrow \infty} \frac{\left(\Omega_{N}, S_{0}{ }^{3} \Omega_{N}\right)}{\left(\Omega_{N}, \Omega_{N}\right)},
$$

where $S_{0}{ }^{3}$ is the third (z-) component of the spin operator at the origin (center of the Cayley tree). As we describe later, we find that the Néel order parameter $m$ is vanishing for $z=3$, and is strictly positive for $z>4$. For $z=4$ numerical calculations indicate the order parameter vanishes, but we have not produced a rigorous proof from these calculations. Note that this is consistent with our conjecture in the previous subsection.

It is also not difficult to show that in the infinite Cayley tree with any $z$ and free boundary conditions, the two point function always decays exponentially as

$$
\left\langle\mathbf{S}_{0} \cdot \mathbf{S}_{x}\right\rangle \sim(-3)^{-|x|},
$$

where $|x|$ is the graph theoretic distance (the number of the bonds between 0 and $x$ ). It may seem that this is inconsistent with the above mentioned Néel order for $z>4$. However, this exponential decay for free boundary conditions is nothing but a pathological feature commonly found in Cayley tree systems. It is due to the exponential increase of the number of neighbors within a fixed distance, and has nothing to do with the existence or absence of Néel order. Moreover, the following quantity indicates the marginality of $z=4$.

$$
\sum(-1)^{|x|}\left\langle\mathbf{S}_{0} \cdot \mathbf{S}_{x}\right\rangle\left\{\begin{array}{lll}
<\infty & \text { for } & z<4, \\
=\infty & \text { for } & z \geqq 4
\end{array}\right.
$$

Finally, we consider the mixed boundary conditions as in Fig. 4.2 which favor the existence of a domain wall between two regions which have opposite Néel orders. The VBS state with these boundary conditions also satisfies the condition $H_{i j} \Omega_{\Lambda}=0$ for any bond $(i, j)$. Unlike the standard models (e.g., Ising or usual Heisenberg models) the domain wall (in the finite system) in the VBS state does not cost any energy.

Let us denote the infinite volume limit of the above state (Fig. 4.2) by $\varrho_{ \pm}$. We can show that, for an even $z>4, \varrho_{ \pm}$contains a domain wall in the sense that $(-1)^{i} \varrho_{ \pm}\left(S_{i}{ }^{3}\right)<0$ when $i$ is in a branch with the plus boundaries, and $(-1)^{i} \varrho_{ \pm}\left(S_{i}^{3}\right)>0$ when $i$ is in a branch with the minus boundaries. Since the state satisfies $\varrho_{ \pm}\left(H_{i j}\right)=0$ for any bond $(i, j)$, the domain wall costs no energy. This raises the possibility that the VBS state with such a zero energy domain wall exists in the regular lattice when the system is in the Neel ordered phase and the dimension is sufficiently high.

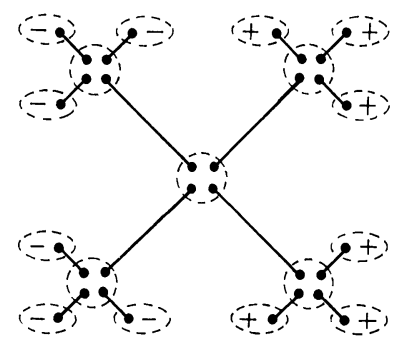

Fig. 4.2. Boundary conditions which favor the existence of a domain wall. $+($ or - ) stands for the three spin $1 / 2$ 's at the boundary 
Derivation of the Results. We now show that the Néel order parameter vanishes for $z=3$ and is strictly positive for $z>4$.

Note that the formula (3.11) for the norm of the VBS state can be easily extended to the general VBS state as follows:

$$
\left(\Omega_{\Lambda}, \Omega_{\Lambda}\right)=\sum_{G^{\prime}, G \subset \mathbb{B}} \prod_{k \in \Lambda} \chi\left(n_{k}\left(G^{\prime}\right)=n_{k}(G)\right) n_{k}(G) !\left(z-n_{k}(G)\right) ! .
$$

Since the Cayley tree contains no loops, the only way to satisfy $n_{k}\left(G^{\prime}\right)=n_{k}(G)$ for all $k$ is to set $G^{\prime}=G$. Thus the above formula simplifies to

$$
\left(\Omega_{N}, \Omega_{N}\right)=\sum_{G \subset \mathbb{B}} \prod_{k \in C_{N}} n_{k}(G) !\left(z-n_{k}(G)\right) !,
$$

where $G$ runs over all subsets of the bonds in $C_{N}$, and $n_{k}(G)$ is the number of bonds in $G$ which are incident to $k$.

Let $B_{M}$ be a branch in the Cayley tree $C_{M}$ where $M$ is an arbitrary integer (Fig. 4.1b). To evaluate the sum (4.8), let us define the following quantities:

$$
\begin{gathered}
Y_{M}=\sum_{G}\left(n_{0}(G)+1\right) !\left(z-n_{0}(G)-1\right) ! \prod_{k \in B_{M} \backslash 0} n_{k}(G) !\left(z-n_{k}(G)\right) !, \\
Z_{M}=\sum_{G} \prod_{k \in B_{M}} n_{k}(G) !\left(z-n_{k}(G)\right) !
\end{gathered}
$$

where 0 denotes the origin of the branch $B_{M}$ (see Fig. 4.1b). Note that $Y_{M}$ and $Z_{M}$ represent the norms of the VBS states on $B_{M}$ which have plus boundary condition and minus boundary condition, respectively, at the origin. Both states have minus (respectively plus) boundary conditions at all the other boundary sites when $M$ is even (respectively odd). Their "initial values" are given by $Y_{1}=(z-1)$ ! and $Z_{1}=z$ !.

The lattice $B_{M+1}$ can be constructed by gluing together one bond and $(z-1)$ copies of $B_{M}$ at a vertex order $z$. Noting that we can assign either $Y_{M}$ or $Z_{M}$ to each $B_{M}$, we get the following recursion equation:

$$
\begin{aligned}
Y_{M+1} & =\sum_{i=0}^{z-1}\left(\begin{array}{c}
z-1 \\
i
\end{array}\right)\left(Y_{M}\right)^{i}\left(Z_{M}\right)^{z-1-i}(i+1) !(z-1-i) ! \\
& =(z-1) ! \sum_{i=0}^{z-1}(i+1)\left(Y_{M}\right)^{i}\left(Z_{M}\right)^{z-1-i} .
\end{aligned}
$$

The recursion equation for $Z$ can be obtained in a similar way,

$$
Z_{M+1}=(z-1) ! \sum_{i=0}^{z-1}(z-i)\left(Y_{M}\right)^{i}\left(Z_{M}\right)^{z-1-i} \text {. }
$$

Let us define a new quantity $W_{M}$ by $W_{M}=Y_{M} / Z_{M}$. From the recursion equations for $Y_{M}$ and $Z_{M}$, we get

$$
W_{M+1}=f_{z}\left(W_{M}\right)
$$

where

$$
f_{z}(W)=\frac{\sum_{i=0}^{z-1}(i+1) W^{i}}{\sum_{i=0}^{z-1}(z-i) W^{i}} .
$$


Therefore, we can find the value of $W_{M}$ by starting from the initial value $W_{1}=1 / z$, and applying the above one-dimensional map $f_{z}(\cdot) M-1$ times. In the infinite volume limit the calculation reduces to the following elementary fixed point analysis.

Note that the map $f_{z}(W)$ satisfies the following properties:

i) $f_{z}(W)$ is continuous for $W>0$.

ii) $f_{z}(W)$ is monotone nondecreasing for $W>0$. (To see this, note that $f_{z}{ }^{\prime}(W)$ $=C \sum_{i, j=1, \ldots, z-1}\left(i^{2}-i j\right) W^{i+j}=C \sum_{i, j=1, \ldots, z-1}\left\{(i-j)^{2} / 2\right\} W^{i+j} \geqq 0$, where $C$ is a positive quantity.)

iii) $1 / z \leqq f_{z}(W) \leqq z$ for $W>0$. (This follows from i) and $f_{z}(0)=1 / z$, $\lim _{W \rightarrow \infty} f_{z}(W)=z$.)

From i)-iii), it follows that the limit

$$
W_{\infty}=\lim _{M \rightarrow \infty} W_{M}=\lim _{M \rightarrow \infty} f_{z}^{M}(1 / z)
$$

always exists and is determined by the fixed point to which the initial value $W=1 / z$ is attracted. [Proof. Let $W_{1}=1 / z$. By iii), $W_{2} \geqq W_{1}$. Because of ii) we then have $W_{3} \geqq W_{2} \Rightarrow W_{4} \geqq W_{3} \Rightarrow$ etc. Therefore, $\left\{W_{M}\right\}$ is a bounded increasing sequence and hence has a limit.]

Clearly, the map $f_{z}(W)$ has $W=1$ as a fixed point. Since the gradient of $f_{z}(W)$ at $W=1$ is $f_{z}^{\prime}(1)=(z-1) / 3$, this fixed point is stable if $z<4$, and unstable if $z>4$. Thus for $z>4$, the initial value $W=1 / z$ cannot be deriven to the fixed point $W=1$. Moreover, from the properties i)-iii), we can conclude the following:

$$
0<W_{\infty}<1 \text { for } z>4 \text {. }
$$

For $z=3$, we can show the following property of $f_{z}(W)$ (by a brute force calculation).

iv) $f_{3}^{\prime \prime}(W) \geqq 0$ holds for $1 / 3 \leqq W \leqq 1$.

Therefore, the initial value $W=1 / 3$ must be attracted to the fixed point $W=1$. Thus we have

$$
W_{\infty}=1 \text { for } z=3 .
$$

For $z=4$, the fixed point $W=1$ is marginally stable. We expect (based on numerical calculations) that the initial value $W=1 / 4$ is driven to this fixed point, and $W_{\infty}=1$ holds.

It is not difficult to find that the expectation value for $S_{0}{ }^{3}$ can be expressed in terms of $W_{\infty}$ as

$$
m_{z}=\lim _{N \rightarrow \infty}\left\langle S_{0}{ }^{3}\right\rangle_{z, N}=\frac{\sum_{i=0}^{z}(z / 2-i)\left(W_{\infty}\right)^{i}}{\sum_{i=0}^{z}\left(W_{\infty}\right)^{i}} .
$$


Substituting the values of $W_{\infty}$ (4.10), (4.11) into the above formula, we get the desired result:

$$
\begin{array}{ll}
m=0 & \text { if } \quad z=3 \\
m>0 & \text { if } \quad z>4
\end{array}
$$

Numerical calculations yield the following values of the (normalized) Néel order parameters:

$$
\begin{aligned}
m / s & =0, & & z=4, \\
& =0.814 \ldots, & & z=5, \\
& =0.902 \ldots, & & z=6 .
\end{aligned}
$$

To get the estimate (4.7) for the correlation function, note that, for free boundary conditions, we have $W_{1}=1$ and thus $W_{M}=1$ for any $M$ and $z$. Therefore, the formula for correlation functions becomes quite simple. Then the desired (4.7) can be derived from a representation similar to (3.14) and a little calculation.

Finally, the result on the zero energy domain wall is a simple consequence of the fact that $W_{\infty}<1$ for the plus boundary conditions, and $W_{\infty}>1$ for the minus boundary conditions if $z>4$.

\section{3. $S U(N)$ VBS State and Random Loop Representation}

Here we will describe a set of heuristic and rigorous results which provide further support for our conjecture (that there is Néel order in the VBS state if the coordination number and dimension are both large and there is none if they are both small) in Sect. 4.1. Two keys for the arguments developed here are $S U(N)$ generalizations of the VBS states, and a random loop representation for the correlation functions. This random loop representation should not be confused with the random walk representation in Sect. 3.3. It is interesting that the VBS state has two natural geometric representations.

First, let us formally extend the VBS state (4.4) to the $S U(n)$ quantum "spin" system [2]. More detailed discussion about the $S U(n)$ generalizations will be given in the next subsection. To get the $S U(n)$ VBS state, we simply let the indices $\alpha_{i j}$ in (4.4) run from 1 to $n$, instead of 1 to 2 . The basis vectors $\psi_{\alpha_{1} \alpha_{2} \ldots \alpha_{z}}$ still satisfy the normalization condition (4.3) where $\delta_{\alpha \beta}$ should be regarded as the $n$ by $n$ unit matrix.

In the one-dimensional model, the calculation of the correlation function presented in Sect. 2.2 generalizes easily to arbitrary $n$. The basic formula for the action of the spin operators (2.15) is still valid for the $S U(n)$ model, if we regard $\boldsymbol{\sigma}_{\alpha}{ }^{\beta}$ as the $S U(n)$ generalization of the Pauli matrices, i.e., a basis of the Lie algebra chosen so that $\operatorname{Tr} \sigma^{a} \sigma^{b}=\left(\sigma^{a}\right)_{\alpha}{ }^{\beta}\left(\sigma^{b}\right)_{\beta}{ }^{\alpha}=2 \delta^{a b}$. Then everything is the same as before but now there is a factor of $n$ for each loop instead of 2 as for $S U(2)$. Thus the normalization of the open chain of length $L$ becomes

$$
\Omega_{\beta}^{\dagger \alpha} \cdot \Omega_{\gamma}{ }^{\delta}=\delta_{\gamma}^{\alpha} \delta_{\beta}^{\delta}\left\{(n+1)^{L}-1\right\} / n+\delta^{\alpha}{ }_{\beta} \delta^{\delta}{ }_{\gamma} .
$$


For the closed chain we obtain $(n+1)^{L}-1+n^{2}$. The correlation function is

$$
\begin{aligned}
\left\langle S^{a}{ }_{0} S^{b}{ }_{r}\right\rangle & =(-1)^{r}(1 / 2)^{2} 2 \delta^{a b}\left[2(n+1)^{L-r}+(n+1)^{L-r-1}+(n+1)^{L-r+1}\right] / n(n+1)^{L} \\
& =(-1)^{r}\left[(n+2)^{2} / 2 n(n+1)\right](n+1)^{-r} \quad(\text { for } r>1)
\end{aligned}
$$

for $a, b=1, \ldots, n^{2}-1$. Note that the correlation length, $1 / \ln (n+1)$, is finite for all $n>0$, and diverges as $n \rightarrow 0$. Thus the $S U(n)$ VBS state in the one-dimensional lattice is in the VBS phase for all $n>0$.

In the Cayley tree model, we can again construct recursion equations for the $n$ quantities corresponding to $Y_{M}, Z_{M}$, and carry out a fixed point analysis similar to (but a little more complicated than) the $S U(2)$ case. Then we find that the trivial fixed point representing the VBS phase [like $W=1$ in the $S U(2)$ case] is stable in the region $(z-1) /(n+1)<1$. Therefore, the $S U(n)$ VBS state is in the VBS phase for $z \geqq n+2$, and in the Neel ordered phase for $z<n+2$. This is consistent with the one-dimensional case where $z=2$ and the critical value of $n$ is zero.

In order to get some idea of the phase diagram for the $S U(n)$ VBS state on regular lattices, we construct a stochastic geometric representation for the correlation functions. The representation is essentially a straightforward extension of the diagramatic method used in Sect. 2.2.

Let $\Lambda$ be an arbitrary finite bipartite lattice with coordination number $z$. To compute the norm of the $S U(n)$ VBS state $\Omega_{\Lambda}$ we apply (4.3) to each site in the representation (4.4) of $\Omega_{\Lambda}$. The result is that $\left(\Omega_{\Lambda}, \Omega_{\Lambda}\right)=\Omega_{\Lambda}^{\dagger} \cdot \Omega_{\Lambda}$ is a sum of $(z !)^{|\Lambda|}$ terms. To obtain a diagramatic representation of this sum we first represent $\Omega_{\Lambda}$ as shown in Fig. 4.3, where we have assigned a direction to each bond in such a way that the bond points from a site in $\Lambda_{A}$ to a site in $\Lambda_{B}$. We represent $\Omega_{A}^{\dagger}$ by the same graph with the direction of every bond reversed. We now place the graphs for $\Omega_{A}$ and $\Omega_{A}{ }^{\dagger}$ on top of each other. One term in the sum (4.3) is represented by pairing each bond coming into the vertex with a bond going out of the vertex. Two bonds that hit a vertex are considered to be attached only if they are paired together. For example, with $z=3$ the 3 ! terms for a single vertex are represented by Fig. 4.4. In this way each term in $\left(\Omega_{\Lambda}, \Omega_{\Lambda}\right)=\Omega_{\Lambda}^{\dagger} \cdot \Omega_{\Lambda}$ is represented by a collection of loops on the lattice. The contribution from a single loop is $\delta_{\alpha_{1}}{ }^{\alpha_{2}} \delta_{\alpha_{2}}{ }^{\alpha_{3}} \ldots \delta_{\alpha_{m}}{ }^{\alpha_{1}}=n$. Thus

$$
\Omega_{\Lambda}^{\dagger} \cdot \Omega_{\Lambda}=\sum_{C} n^{\# C}
$$

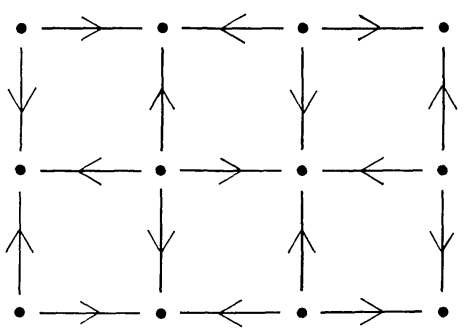

Fig. 4.3.

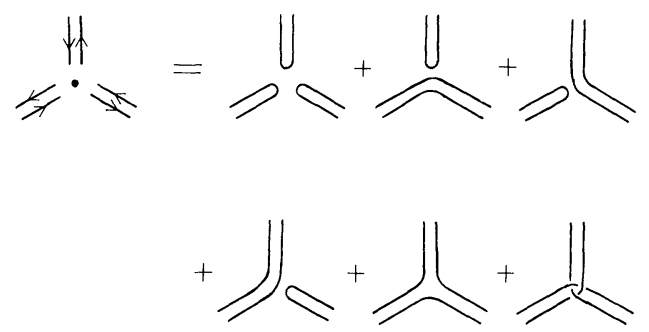

Fig. 4.4.

Fig. 4.3. Diagramatic representation of $\Omega_{\Lambda}$

Fig. 4.4. Diagramatic rule used in calculating the overlaps 
where $\# C$ is the number of distinct loops in the configuration $C$, and the sum is over all configurations of loops on the lattice with the property that each bond in the lattice is covered exactly twice by $C$.

To get the expression for the correlation functions, we generalize (2.15) to general spin $s$ as

$$
\mathbf{S} \psi_{\alpha_{1} \alpha_{2} \ldots \alpha_{z}}=-\frac{1}{2} \sum_{k=1}^{z} \boldsymbol{\sigma}_{\alpha_{k}}{ }^{\beta} \psi_{\alpha_{1} \alpha_{2} \ldots \hat{\alpha}_{k} \ldots \alpha_{z} \beta},
$$

where $\hat{\alpha}_{k}$ is an omitted index, and $\boldsymbol{\sigma}_{\alpha}{ }^{\beta}$ again denotes the $S U(n)$ version of the Pauli matrices. Combining this formula with (4.4), we can again express the unnormalized correlation functions in terms of the random loop configurations as

$$
\Omega_{\Lambda}{ }^{\dagger} \cdot \mathbf{S}_{i} \cdot \mathbf{S}_{j} \Omega_{\Lambda}=(-1)^{i-j} / 4 \sum_{\substack{b \ni i \\ b^{\prime} \ni j}} \sum_{C} \chi\left(b \text { and } b^{\prime} \text { belong to the same loop }\right) n^{\# C} .
$$

Here the characteristic function $\chi(A)$ equals 1 if $A$ is true, and 0 if $A$ is false. $b$ (respectively $b^{\prime}$ ) runs over the bonds in the graph of $\Omega_{\Lambda}$ which contain $i$ (respectively $j$ ). The prefactor $(-1)^{i-j}$ is 1 if $i$ and $j$ belong to the same sublattice, and -1 otherwise. We have assumed that $i$ and $j$ are separated by a distance $>1$.

From (4.12) and (4.13) we get the following random loop representation for the correlation function.

$$
\left\langle\mathbf{S}_{i} \cdot \mathbf{S}_{j}\right\rangle_{\Lambda, n}=(-1)^{i-j} / 4 \sum_{\substack{b \ni i \\ b^{\prime} \ni j}} \operatorname{Prob}_{\Lambda, n}\left(b \text { and } b^{\prime}\right. \text { belong to the same loop). }
$$

Here the probability for the basic event that a particular random loop configuration $C$ is present is defined to be

$$
\operatorname{Prob}_{\Lambda, n}(C)=n^{\# C} / \sum_{C^{\prime}} n^{\# C^{\prime}} .
$$

Note that, in the above definition, the logarithm of $n$ can be regarded as the chemical potential for the loops in our stochastic geometric system. By (4.14) and (4.15), we are now able to extend the correlation function for $S U(n)$ VBS state to noninteger (complex!) values of $n$. In the following analysis, we regard (4.14) and (4.15) as our starting points, and consider positive but not necessarily integer $n$.

For large values of $n$, the configurations with many loops (as in Fig. 4.5a) are favored by (4.15). We expect that the random loop system is in the nonpercolating phase where the loops are localized and have finite size (with probability one). In particular, the probability that two sufficiently separated bonds belong to the same loop is expected to decay exponentially with the distance between the bonds. Thus the $S U(n)$ VBS state should be in the VBS phase which has short range correlations.

For small values of $n$, the configurations with large loops (as in Fig. 4.5b) are the main contribution to (4.13), (4.14). Then the random loop system (in the infinite volume limit) may be in the percolating phase where a (unique) infinite loop appears in the configuration (with probability one). If the probability that a bond belongs to the infinite loop is strictly positive, (4.14) suggests that the two point correlation function $\left|\left\langle\mathbf{S}_{i} \cdot \mathbf{S}_{j}\right\rangle\right|$ is bounded below by this probability squared. 

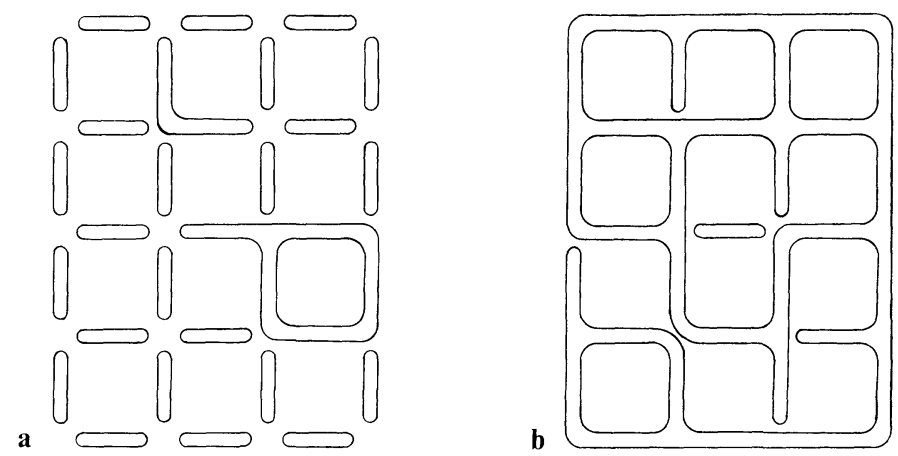

Fig. 4.5. Typical diagrams which contribute to $\Omega_{A}{ }^{\dagger} \cdot \Omega_{\Lambda}$ for a large $n$ (nonpercolating phase) and b small $n$ (percolating phase)

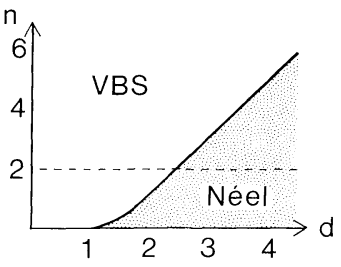

Fig. 4.6. Conjectured phase diagram for the $S U(n)$ VBS states

Therefore, the $S U(n)$ VBS state is expected to be in the Néel ordered phase. Of course, this is not true for the one-dimensional chain, where the VBS phase exist for all $n>0$. However, this simply reflects the special character of the marginal onedimensional lattice, where there are relatively few infinite loops.

In the general infinite lattice, therefore, we conjecture that there exists a critical value $n_{c}(\geqq 0)$. The $S U(n)$ VBS state is in the VBS phase (which corresponds to the nonpercolating phase) for $n>n_{c}$, and is in the Néel ordered phase (which corresponds to the percolating phase) for $n<n_{c}$. The critical value $n_{c}$ is exactly known only for the Cayley tree model where $n_{c}=z-2$ and for the one-dimensional lattice where $n_{c}=0$. We expect that this Cayley tree value becomes reliable in higher dimensions. In Fig. 4.6, we summarize our conjectured phase diagram for the $S U(n)$ VBS state on the $d$-dimensional hypercubic lattice. This phase diagram, when restricted to $n=2$, again suggests the existence of a transition from the VBS phase to the Néel ordered phase as the dimension is increased. Thus it provides further support for our conjecture in Sect. 4.1.

It is desirable to have rigorous arguments which support the above heuristic discussion about the $S U(n)$ VBS state. At present we can only prove the following proposition which confirms our picture for sufficiently large values of $n$. The proof of the existence of Neel order for sufficiently small $n$ is probably difficult since it involves continuous symmetry breaking. We also note that Theorem 3.1 proves the upper bound $n_{c}<2$ for any bipartite lattice with coordination number three.

Proposition 4.1. Consider an arbitrary (infinite) bipartite lattice with coordination number $z$. If $n>(z !)^{2(z-1)}$, the $S U(n) V B S$ state on the lattice is in the VBS phase, i.e., 
every truncated correlation function decays exponentially, and there is no Néel order.

Proof. Consider a finite lattice, and let $l$ be a loop which contains a fixed bond $b_{0}$ in the graph of $\Omega_{\Lambda}$. The statistical weight for a single loop $l$ is defined as

$$
\omega(l)=\sum_{C \ni l} n^{\# C} / \sum_{C} n^{\# C} .
$$

In the following we prove that $\omega(l)$ can be bounded uniformly in the lattice size as

$$
\omega(l) \leqq n\left\{(z-1) !(z !)^{z-2} / \sqrt{n}\right\}^{|l|},
$$

where $|l|$ is the length (number of bonds) of $l$. From this bound and the representation (4.14), we get the following upper bound for the two point correlation function provided that $n>(z !)^{2(z-1)}$ :

$$
\begin{aligned}
\left\langle\mathbf{S}_{i} \cdot \mathbf{S}_{j}\right\rangle & \leqq z^{2} \operatorname{Prob}(|l| \geqq 2(|i-j|-2)) \\
& \leqq \sum_{k=2(|i-j|-2)}^{\infty} z^{k} n\left\{(z-1) !(z !)^{z-2} / \sqrt{n}\right\}^{k}=C \exp \left(-|i-j| / \xi_{0}\right) .
\end{aligned}
$$

Here $0<C, \xi_{0}<\infty$ are independent of the lattice size. We have bounded the number of the loops with $|l|=k$ by $z^{k}$ in the above.

To prove the asserted bound for $\omega(l)$, consider an arbitrary loop $l$ and denote by $\hat{l}$ the set of all the lattice bonds which belong to or are incident to $l$. Given a loop configuration $C \ni l$, we replace the loop configuration on $\hat{l}$ by a collection of minimum single bond loops to construct a new configuration $\hat{C}$. Clearly, $\hat{C}$ contains at least $|l| / 2-1$ more loops than $C$ does. Noting that at most $(z-1) !^{|l|}(z !)^{(z-2)|l|}$ different $C$ can be mapped into a single $\widehat{C}$ (because of the redundant freedom in $\hat{l}$ ), we get

$$
n^{|l| / 2-1} \sum_{C \ni l} n^{\# C} /(z-1) !|l|(z !)^{(z-2)|l|} \leqq \sum_{C} n^{\# C},
$$

which implies the desired bound for $\omega(l)$. The exponential decay of every truncated correlation function and the absence of Néel order can be proved by the same estimates.

\section{4. $S U(n)$ Quantum "Spin" System}

In the present subsection we amplify the discussions of the $S U(n)$ VBS state in the previous subsection by presenting some details of the $S U(n)$ quantum "spin" systems which were first introduced in [2].

Consider the simplest $s=1 / 2$ case. As we have noted several times, we denote the standard $S^{3}$ eigenstates $(+)$ and $(-)$ by $\psi_{\alpha}, \alpha=1,2$, respectively. To generalize this basis from $S U(2)$ to $S U(n)$, we simply let the index $\alpha$ run from 1 to $n$. Then the basis for a finite bipartite lattice again consists of the simple tensor product of $\psi_{\alpha}$ on one sublattice and $\psi^{\alpha}$ on the other sublattice. This corresponds to putting the fundamental representation of $S U(n)$ on one sublattice, and its conjugate representation on the other. Again the $S U(n)$ singlet (valence bond) can be simply represented as $\psi_{\alpha}^{\alpha}\left(=\psi_{\alpha} \otimes \psi^{\alpha}\right)$. 
To get the $S U(n)$ generalization of the Hamiltonian, it is useful to represent the action of the Hamiltonian in terms of the valence bond basis. Note that in the $S U(2)$ case, the $s=1 / 2$ Heisenberg Hamiltonian (with convenient constants inserted),

$$
H=2 \mathbf{S} \cdot \mathbf{S}^{\prime}-1 / 2
$$

acting on a pair of neighboring spins gives

$$
H \psi_{\alpha}{ }^{\beta}=-\delta_{\alpha}{ }^{\beta} \psi_{\gamma}{ }^{\gamma}
$$

Written in this way an obvious generalization from $S U(2)$ to $S U(n)$ suggests itself. The Hamiltonian for the $S U(n)$ model is still defined by the above equation if we allow the indices $\alpha$ to run from 1 to $n$. The action of $H$ on a valence bond state can be obtained by observing that

$$
H \psi_{\alpha}^{\alpha}=-n \psi_{\alpha}^{\alpha} \text { and } H_{23} \psi_{\alpha \beta}^{\alpha \beta}=-\psi_{\alpha \beta}{ }^{\beta}{ }^{\alpha} .
$$

(In the latter equation $H_{23}$ denotes the term in $H$ acting on the second and third sites.) This can be represented graphically as the following:

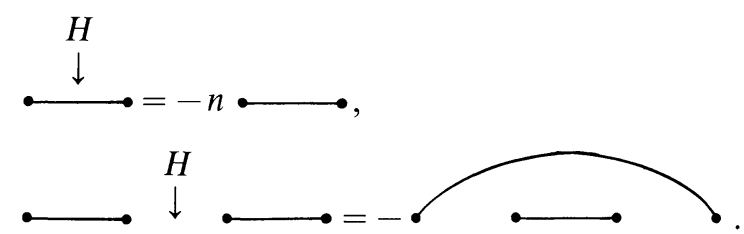

In the $s=1$ case, we again let the indices in the basis vector $\psi_{\alpha \beta}$ run from 1 to $n$. We now have the symmetric tensor representation $\psi_{\alpha \beta}$ on odd sites and its conjugate representation $\psi^{\alpha \beta}$ on even sites. The Heisenberg Hamiltonian

$$
H_{1}=2 \mathbf{S} \cdot \mathbf{S}^{\prime}-2
$$

acting on $\psi$ gives

$$
\begin{aligned}
H_{1} \psi_{\alpha_{1} \alpha_{2}}{ }^{\alpha_{3} \alpha_{4}}= & -\delta_{\alpha_{1}}^{\alpha_{3}} \psi_{\beta \alpha_{2}}{ }^{\beta \alpha_{4}}-\delta_{\alpha_{1}}^{\alpha_{4}} \psi_{\beta \alpha_{2}}{ }^{\alpha_{3} \beta} \\
& -\delta_{\alpha_{2}}{ }^{\alpha_{3}} \psi_{\alpha_{1} \beta}{ }^{\beta \alpha_{4}}-\delta_{\alpha_{2}}^{\alpha_{4}} \psi_{\alpha_{1} \beta}{ }^{\alpha_{3} \beta} .
\end{aligned}
$$

We may again generalize the Hamiltonian to $S U(n)$ by simply letting the indices run from 1 to $n$. The action of $H_{1}$ on the VBS state can be represented graphically as follows.

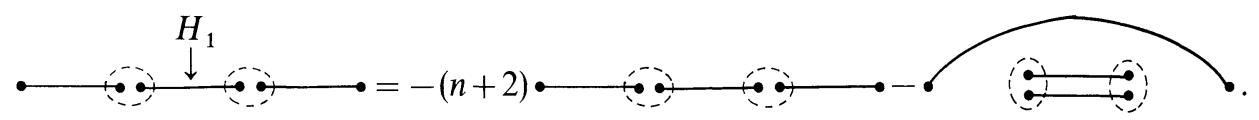

In the $S U(2)$ case the biquadratic Hamiltonian

$$
H_{2} \equiv 2\left(\mathbf{S} \cdot \mathbf{S}^{\prime}\right)^{2}-2
$$

produces double bonds on nearest neighbor links

$$
H_{2} \psi_{\alpha \beta}^{\gamma \delta}=\left(\delta_{\alpha}^{\gamma} \delta_{\beta}^{\delta}+\delta_{\alpha}^{\delta} \delta_{\beta}^{\gamma}\right) \psi_{\varepsilon \varrho}^{\varepsilon \varrho} .
$$


Again we extend this definition of $H_{2}$ to $S U(n)$. The action on the VBS state can be represented as follows:

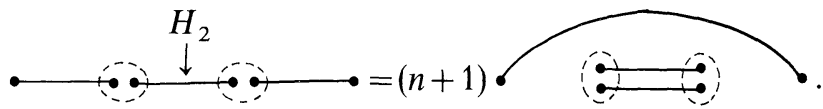

Consider the one-dimensional $S U(n)$ quantum "spin" system with " $s=1$ " and the general bilinear-biquadratic Hamiltonian

$$
H=\sum_{i} \frac{1}{n} H_{1}\left(\mathbf{S}_{i}, \mathbf{S}_{i+1}\right)-\frac{\beta}{n^{2}} H_{2}\left(\mathbf{S}_{i}, \mathbf{S}_{i+1}\right) .
$$

(The factors of $n$ are inserted so that the spacing of eigenvalues of $H$ remains finite as $n \rightarrow \infty$.) The $S U(n)$ VBS state introduced in the previous subsection becomes the exact ground state at $\beta=-n /(n+1)$. This can be seen by considering the action of $H$ using the above graphical rules, or by observing that this Hamiltonian is a projection operator onto the $S U(n)$ representation which generalizes $S=1$, namely the symmetrized traceless tensor with two upper and two lower indices. It is worth noting that, in the limit $n \rightarrow \infty$, the exact ground states of (4.16) can be constructed entirely out of nearest neighbor valence-bonds for arbitrary $\beta$. For $\beta \leqq 0$ the ground state is the VBS state, and for $\beta \geqq 0$ the ground state is the two dimerized states which have double valence-bonds on every other bond. This provides a support for the phase transition picture for the ordinary spin 1 chain described in the introduction and [1].

$S U(n)$ quantum "spin" systems with higher " $s$ " can be defined in a similar way by putting a variable $\psi_{\alpha_{1} \alpha_{2} \ldots \alpha_{2 s}}$, which transforms under the symmetric $2 s$-tensor representation of $S U(n)$, on one sublattice and its conjugate representation $\psi^{\alpha_{1} \alpha_{2} \ldots \alpha_{2 s}}$ on the other sublattice. When the coordination number $z$ equals $2 s$, a Hamiltonian in which the $S U(n)$ VBS state is an exact ground state can be constructed. It is a projection onto the "maximal" representation contained in the product of these two representations, namely the symmetric, traceless tensor with $z$-upper and $z$-lower indices.

\section{Energy Gap in the Majumdar-Ghosh Model}

\subsection{The Model and the Ground States}

As in Sect. 2, we consider a one-dimensional lattice, but $\mathbf{S}_{i}$ now denotes the spin operator for $\operatorname{spin} s=1 / 2$ at site $i$. The Hamiltonian for the Majumdar-Ghosh model is

$$
H=\sum_{i} H_{i} \quad \text { with } H_{i}=P_{3 / 2}\left(\mathbf{S}_{i}+\mathbf{S}_{i+1}+\mathbf{S}_{i+2}\right),
$$

where $P_{3 / 2}(\mathbf{S})$ is the orthogonal projection onto the subspace with $\mathbf{S}^{2}=(3 / 2)(1+3 / 2)(\operatorname{spin} 3 / 2)$. This Hamiltonian can be written as the usual spin $1 / 2$ Heisenberg model plus a next nearest neighbor term by using the identity

$$
P_{3 / 2}\left(\mathbf{S}_{i}+\mathbf{S}_{i+1}+\mathbf{S}_{i+2}\right)=\frac{2}{3}\left(\mathbf{S}_{i} \cdot \mathbf{S}_{i+1}+\mathbf{S}_{i} \cdot \mathbf{S}_{i+2}+\mathbf{S}_{i+1} \cdot \mathbf{S}_{i+2}\right)+\frac{1}{2} .
$$


In order to describe the ground states of the system, we again make use of an $S U(2)$ notation similar to that of the previous sections. For a single site we denote the standard $S^{3}= \pm 1 / 2$ basis $\{(+),(-)\}$ by

$$
\psi_{1}=(+), \quad \psi_{2}=(-) \text {. }
$$

We also make use of the raising convention $\psi^{\alpha}=\varepsilon^{\alpha \beta} \psi_{\beta}$ and the summation convention for repeated upper and lower indices. For a system on a finite chain $\{1,2, \ldots, L\}$, we use the basis which consists of simple tensor products of $\psi_{\alpha}$ 's as in Sect. 2. In particular, the following choice is convenient for our purposes.

$$
\psi_{\alpha_{1}}^{\alpha_{2} \ldots \alpha_{L-1}{ }_{\alpha_{L}}}=\psi_{\alpha_{1}} \otimes \psi^{\alpha_{2}} \otimes \ldots \otimes \psi^{\alpha_{L-1}} \otimes \psi_{\alpha_{L}} .
$$

Each $\alpha_{i}$ can be 1 or 2 . This equation applies for the case of odd $L$. If $L$ is even the $\alpha_{L-1}$ should be a lower index and the $\alpha_{L}$ should be an upper indice. These states provide us with a complete set of states for the finite chain.

The ground states of the Majumdar-Ghosh model are easily written in this notation. For odd $L$ there are four linearly independent ground states given by

$$
\begin{aligned}
& \Omega^{(1)}{ }_{\alpha}=\psi_{\alpha_{1}}{ }^{\alpha_{1}} \alpha_{\alpha_{2}} \alpha_{2} \cdots \alpha_{(L-1) / 2}{ }^{\alpha_{(L-1) / 2}}{ }_{\alpha}, \\
& \Omega^{(2)}{ }_{\alpha}=\psi_{\alpha}^{\alpha_{1}}{ }_{\alpha_{1}} \alpha_{\alpha_{2}}{ }^{\alpha_{(L-1) / 2}}{ }_{\alpha_{(L-1) / 2}},
\end{aligned}
$$

with $\alpha=1$ or 2 . For even $L$ there are five linearly independent ground states given by

$$
\begin{aligned}
& \Omega^{(1)}=\psi_{\alpha_{1}}{ }^{\alpha_{1}} \alpha_{2} \alpha_{2} \cdots_{\alpha_{L / 2}}{ }^{\alpha_{L / 2}}, \\
& \Omega_{\alpha}^{(2) \beta}=\psi_{\alpha}{ }_{\alpha_{1}}^{\alpha_{1} \alpha_{\alpha_{2}}} \ldots^{\alpha_{(L-2) / 2}}{ }_{\alpha_{(L-2) / 2}}^{\beta} \text {, }
\end{aligned}
$$

with $\alpha, \beta=1$ or 2 .

As is clear from the formulae, we get two different infinite volume ground states from these states. If we again denote the singlet pairs by line segments, the ground states (5.4) and (5.5) can be represented diagramatically as shown in Fig. 5.1. Unlike the valence-bond solid states, the valence bonds now cover only half of the lattice. Moreover, the ground states have period 2, and thus break the translational symmetry of the Hamiltonian.

Also note that these dimerized ground states are mere tensor products of local singlet pairs. Therefore, correlation functions for these dimerized states are just

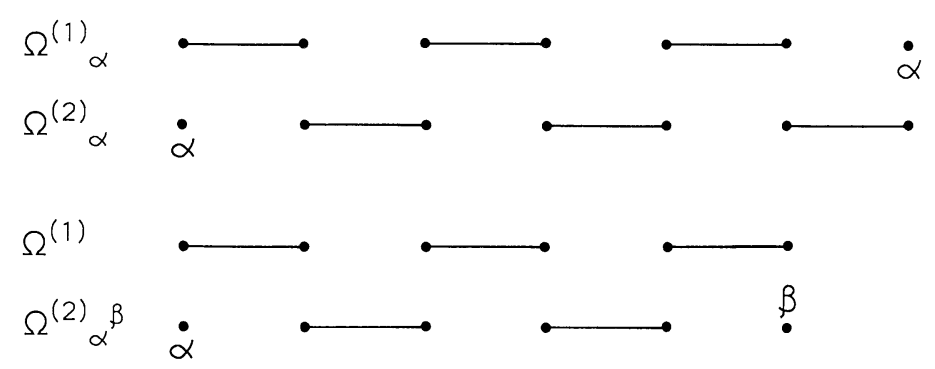

Fig. 5.1. The ground states of the Majumdar-Ghosh model 
trivial combinations of singlet pair correlation functions. In particular, the two point correlation function $\left\langle\mathbf{S}_{i} \cdot \mathbf{S}_{j}\right\rangle$ vanishes whenever $|i-j| \geqq 2$.

To see that the states in (5.4) and (5.5) are ground states of (5.1), observe that the restriction of each state to any three adjacent sites contains a singlet pair. Therefore, the total spin of these three sites is always $1 / 2$. Then the projection operator representation (5.1) of the Hamiltonian immediately implies that the state has energy zero.

This model and the above states were first discovered by Majumdar and Ghosh $[31,32]$, and in $[15]$ it was proved that these states were ground states. Some exact excited states were constructed in [19,39]. For some related results see [40]. We will show that the states in (5.4) and (5.5) are the only ground states and that there is a gap in the spectrum of the Hamiltonian.

\subsection{The Energy Gap}

The techniques used in Sect. 2.3 can also be used to prove there is a gap in the spectrum of the Majumdar-Ghosh Hamiltonian. This subsection is devoted to the existence of the gap in finite chains. The infinite volume result appears at the end of Sect. 5.3.

Theorem 5.1. Consider the Hamiltonian

$$
H_{1, L}=\sum_{i=1}^{L-2} H_{i}
$$

with $H_{i}$ defined by (5.1). There is a positive constant $\varepsilon$ which does not depend on L such that

$$
H_{1, L} \geqq \varepsilon P_{L},
$$

where $P_{L}$ is the projection onto the orthogonal complement of the ground states of $H_{1, L}$.

As in Sect. 2 we define $Q_{n}$ to be the projection onto the subspace of states which are ground states for $H_{1, n}$, and define $P_{n}$ to be $1-Q_{n}$. Any ground state for $H_{1, n+1}$ is also a ground state for $H_{1, n}$. Thus the $Q_{n}$ are a decreasing sequence of projections and the $P_{n}$ are an increasing sequence of projections. This was the only property of the model that we used in proving Lemmas 2.2 and 2.3 and in using Lemmas 2.2, 2.3, and 2.4 to prove Theorem 2.1. Thus all we have to do in the present case is to prove an analog of Lemma 2.4 .

There is one minor difference between Sect. 2.3 and the present model, namely the dimension of $Q_{n}-Q_{n+1}$. As we will show in Sect. 5.3 the ground states defined by (5.4) and (5.5) are the only ground states. Hence $Q_{n}$ restricted to sites 1 to $n$ has dimension 5 if $n$ is even and 4 if $n$ is odd. Thus $Q_{n}-Q_{n+1}$ restricted to sites 1 to $n+1$ has dimension $5 \times 2-4=6$ if $n$ is even and $4 \times 2-5=3$ if $n$ is odd. As in Sect. 2 we let $\varphi_{n+1}^{i}$ be an orthonormal basis for $Q_{n}-Q_{n+1}$ on sites 1 to $n+1$ and define $\varepsilon(l)$ as before:

$$
\varepsilon(l)=\sup _{n} \max _{i}\left\|Q_{n-l+1, n+1} \varphi_{n+1}^{i}\right\|^{2}
$$


Note that in Sect. 2.3 it would have been sufficient to prove Lemma 2.4 only for even $l$. Here we will only prove the lemma for even $l$ since it reduces the number of cases we must consider. The analog of Lemma 2.4 is the following.

Lemma 5.2. There is a constant $c$ such that for all even $l$

$$
\varepsilon(l) \leqq c 2^{-l / 2} .
$$

Proof. We begin by computing the norms and overlaps of the various ground states. As in Sect. 2 this computation may be done in a diagramatic fashion, but here each calculation only involves one diagram. For example, the overlap of $\Omega^{(1)}{ }_{\alpha}$ and $\Omega^{(2)}{ }_{\beta}$ is given by the diagram in Fig. 5.2. This diagram simply equals $\delta^{\beta}{ }_{\alpha}$. The norm of $\Omega^{(1)}{ }_{\alpha}$ is given by a sequence of small loops with one loop for every two sites. Since each loop gives a factor of 2 , the square of the norm of $\Omega^{(1)}{ }_{\alpha}$ is $2^{(L-1) / 2}$. The other norms and overlaps may be computed in the same fashion.

We consider first the case that $n$ is odd. Every state we encounter in the proof will be a ground state for $H_{1, n-l}$ and a ground state for $H_{n-l+1, n}$. Thus it is convenient to introduce the states $\Omega_{\alpha \gamma}^{(12) \beta \delta}, \Omega_{\alpha}^{(21) \beta}, \Omega_{\alpha}^{(11) \beta}$, and $\Omega_{\alpha \gamma}^{(22){ }_{\alpha} \delta}$ which are defined diagramatically by Fig. 5.3.

Let $\varphi$ be one of the $\varphi_{n+1}^{i}$. Then $\varphi$ is a ground state of $H_{1, n}$ and so is a linear combination of $\Omega_{\alpha \gamma}^{(12) \alpha \delta}$ and $\Omega_{\gamma}^{(21) \delta}$ :

$$
\varphi=\Omega_{\alpha \gamma}^{(12) \alpha \delta} A_{\delta}^{\gamma}+\Omega_{\gamma}^{(21) \delta} B_{\delta}^{\gamma} .
$$

The norms of the states in Fig. 5.3 are either $2^{(n-1) / 4}$ or $2^{(n-3) / 4}$. The overlap of $\Omega^{(12){ }_{\alpha \gamma}{ }^{\delta}}$ and $\Omega^{(21)}{ }_{\gamma}^{\delta}$ is either 0 or 1 . Since $\varphi$ is defined so that it has norm 1 , it follows that

$$
\begin{array}{ll}
\left|A_{\delta}^{\gamma}\right|=O\left(2^{-n / 4}\right) & \text { for any } \gamma, \delta, \\
\left|B_{\delta}^{\gamma}\right|=O\left(2^{-n / 4}\right) & \text { for any } \gamma, \delta .
\end{array}
$$

$\varphi$ is orthogonal to any state which is a ground state for $H_{1, n+1}$. These ground states include $\Omega_{\alpha \gamma}^{(12) \alpha \gamma}$ and $\Omega_{\alpha}^{(21) \beta}$. We compute their inner products with $\varphi$ and

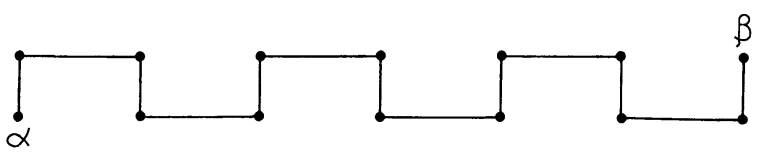

Fig. 5.2. The one diagram which contributes to the overlap of $\Omega^{(1)}{ }_{\alpha}$ and $\Omega^{(2)}{ }_{\beta}$

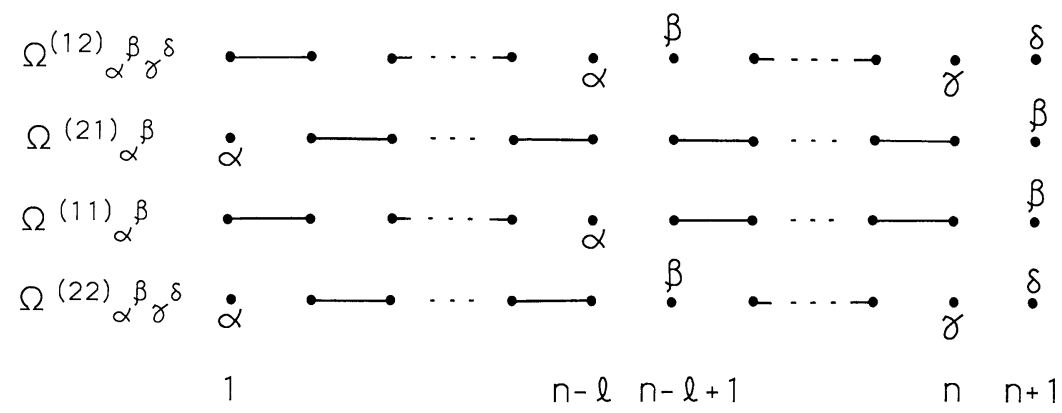

Fig. 5.3. The states which are used in the proof of the existence of a gap 
find

$$
\begin{gathered}
0=\Omega_{\delta}^{\dagger(21) \gamma} \cdot \varphi=A_{\delta}^{\gamma}+2^{(n-1) / 2} B_{\delta}^{\gamma}, \\
0=\Omega_{\alpha \beta}^{\dagger(12) \alpha \beta} \cdot \varphi=A_{\gamma}^{\gamma} 2^{(n-1) / 2}+B_{\gamma}^{\gamma} .
\end{gathered}
$$

Equations (5.9) and (5.8a) imply

$$
\left|B_{\delta}^{\gamma}\right|=O\left(2^{-3 n / 4}\right) \text { for any } \gamma, \delta .
$$

Equations (5.10) and (5.11) imply

$$
\left|A_{\gamma}^{\gamma}\right|=O\left(2^{-5 n / 4}\right) \text {. }
$$

Our goal is to show that $\left\|Q_{n-l+1, n+1} \varphi\right\|^{2}$ is $O\left(2^{-l / 2}\right)$. By (5.7) $\varphi$ is a sum of two terms. The norm of the second term is

$$
\left\|\Omega_{\gamma}^{(21) \delta} B_{\delta}^{\gamma}\right\|=O\left(2^{-3 n / 4}\right) \max _{\gamma, \delta}\left\|\Omega_{\gamma}^{(21) \delta}\right\|=O\left(2^{-n / 2}\right),
$$

where we have used (5.11) and $\left\|\Omega_{\gamma}^{(21)} \delta\right\|=2^{-(n-1) / 4}$. Since $n \geqq l$ this implies the square of the norm is $O\left(2^{-l}\right)$. Thus letting $\varphi^{\prime}$ denote the first term in (5.7), i.e., $\varphi^{\prime}=\Omega^{(12) \alpha}{ }_{\alpha \gamma}^{\delta} A_{\delta}^{\gamma}$, it suffices to show $\left\|Q_{n-l+1, n+1} \varphi^{\prime}\right\|^{2}=\left(\varphi^{\prime}, Q_{n-l+1, n+1} \varphi^{\prime}\right)$ is $O\left(2^{-l / 2}\right)$.

$\varphi^{\prime}$ is a ground state of $H_{1, n-l}$ and $Q_{n-l+1, n+1}$ commutes with this Hamiltonian, so $Q_{n-l+1, n+1} \varphi^{\prime}$ is a ground state of $H_{1, n-l}$. The vector $Q_{n-l+1, n+1} \varphi^{\prime}$ is trivially a ground state of $H_{n-l+1, n+1}$, so it is a linear combination of $\Omega_{\alpha{ }_{\gamma}(12)}{ }^{\gamma}, \Omega^{(21)}{ }_{\alpha}, \Omega^{(11)}{ }_{\alpha}$, $\Omega_{\alpha \gamma}^{(22) \beta}$. Each of these states has norm $2^{(n-1) / 4}$ and their overlaps are 0 or 1 . Hence the coefficients in the linear combination must all be $O\left(2^{-n / 4}\right)$. Thus it suffices to show the inner product of $\varphi^{\prime}$ with each of these states is $O\left(2^{-l / 2+n / 4}\right)$.

The inner product with $\Omega^{(12) \beta}{ }_{\alpha}{ }^{\gamma}$ is $\delta^{\beta}{ }_{\alpha} A^{\gamma}{ } 2^{(n-3) / 2}=O\left(2^{-3 n / 4}\right) \leqq O\left(2^{-l / 2+n / 4}\right)$ by (5.11). The inner product with $\Omega^{\alpha(2)}{ }_{\alpha} \gamma^{\gamma}$ also involves $A^{\gamma}{ }_{\gamma}$ and is sufficiently small. The inner product with $\Omega^{(11) \beta}{ }^{\beta}$ is $A_{\alpha}^{\alpha} 2^{\left({ }^{(n-l-1) / 2}\right.}=O\left(2^{n / 4-l / 2}\right)$ by (5.8a). The overlap with $\Omega^{(21)}{ }_{\alpha}^{\beta}$ is $A_{\alpha}{ }^{\beta}=O\left(2^{-n / 4}\right) \leqq O\left(2^{\alpha / 4-l / 2}\right)$ since $n \geqq l$.

If $n$ is even the proof is essentially the same. We simply modify Fig. 5.3 by adding a site on the left in an obvious way.

\subsection{The Infinite Chain}

In this section we show that in the infinite volume limit there are exactly two ground states and each of them has a gap. We denote the four ground states on $\{-L,-L+1, \ldots, L\}$ by $\Omega^{(1, L)}$ and $\Omega^{(2, L)}, \alpha=1,2$.

Theorem 5.3. Let $A$ be a local operator. Then for $i=1,2$, the expectation value

$$
\left.\omega^{i}(A)=\frac{\left(\Omega^{(i, L)}{ }_{\alpha}, A \Omega^{(i, L)}\right)}{\left(\Omega_{\alpha}^{(i, L)}{ }_{\alpha}, \Omega^{(i, L)}{ }_{\alpha}\right)} \quad \text { (no sum over } \alpha\right)
$$

is independent of $L$ and $\alpha$ provided that $L$ is large enough that the support of $A$ is contained in the interval $\{-L+1,-L+2, \ldots, L-1\}$. Equation (5.13) defines two distinct infinite volume ground states $\omega^{1}$ and $\omega^{2}$. If $\varrho$ is an infinite volume state which is a ground state in the sense that $\varrho\left(H_{i}\right)=0$ for all $i$, then $\varrho=c \omega^{1}+(1-c) \omega^{2}$ for some constant $c, 0 \leqq c \leqq 1$.

The key ingredient in the proof is a finite volume result. 
Lemma 5.4. Let $\varphi$ be a state on $\{-L,-L+1, \ldots, L\}$ which satisfies $H_{i} \varphi=0$ for $i=-L, \ldots, L-2$. Then $\varphi$ can be written as

$$
\varphi=A^{\alpha} \Omega_{\alpha}^{(1, L)}+B^{\alpha} \Omega_{\alpha}^{(2, L)}
$$

for some complex coefficients $A^{\alpha}$ and $B^{\alpha}$.

Proof of Theorem 5.3. Since the states $\Omega^{(i, L)}$ are tensor products of states on two sites, the first claim in the theorem is trivial. The states $\omega^{1}$ and $\omega^{2}$ are clearly distinct.

Let $\varrho$ be an infinite volume ground state. Choose a local observable $B$ with $\omega^{1}(B)=1$ and $\omega^{2}(B)=0$, e.g., $B=-(4 / 3) \mathbf{S}_{1} \cdot \mathbf{S}_{2}$. Let $c=\varrho(B)$. It is easily shown that

$$
\lim _{L \rightarrow \infty} \frac{\left(\Omega^{(i, L)}, A \Omega^{(j, L)}\right)}{\left\|\Omega^{(i, L)}{ }_{\alpha}\right\|\left\|\Omega^{(j, L)}{ }_{\beta}\right\|}=0
$$

for any local observable $A$ unless $i=j$ and $\alpha=\beta$. Lemma 5.4 and Eq. (5.15) imply $\varrho=c \omega^{1}+(1-c) \omega^{2}$ by an argument similar to the proof of Theorem 2.7.

Proof of Lemma 5.4. We proceed by induction in the length of the chain. For 3 sites the ground state must be of the form

$$
\Omega=\psi_{\alpha \beta}^{\alpha} A^{\beta}+\psi_{\alpha \beta}^{\beta} B^{\alpha} .
$$

(This can be seen by counting dimensions. The above equation yields four linearly independent ground states, and the subspace of total spin $1 / 2$ for the tensor product of three spin 1/2's has dimension four.) For 4 sites any ground state $\Omega$ can be written

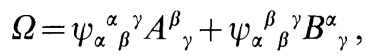

since it is a ground state for the first three sites, and

$$
\Omega=\psi_{\alpha}^{\beta} \gamma^{\gamma} C^{\alpha}{ }_{\beta}+\psi_{\alpha \beta}^{\beta}{ }^{\gamma} D_{\gamma}^{\alpha},
$$

since it is a ground state for the last three sites.

We need to show that

$$
A_{\gamma}^{\beta}=C_{\gamma}^{\beta}=E \delta_{\gamma}^{\beta} .
$$

We again establish these equations by considering various components of $\Omega$. We list the component considered and the corresponding equation.

$$
\begin{array}{ll}
\psi_{1{ }_{2}{ }^{1}:}: & B^{1}{ }_{1}=D^{1}{ }_{1} . \\
\psi_{2}{ }^{1}{ }^{2}: & B^{2}{ }_{2}=D^{2}{ }_{2} . \\
\psi_{1}{ }^{1}{ }_{1}: & A^{1}{ }_{1}+B^{1}{ }_{1}=C^{1}{ }_{1}+D^{1}{ }_{1} . \\
\psi_{2}{ }^{2}{ }^{2}: & A^{2}{ }_{2}+B^{2}{ }_{2}=C^{2}{ }_{2}+D^{2}{ }_{2} . \\
\psi_{1}{ }^{1}{ }^{2}: & A^{2}{ }_{2}=C^{1}{ }_{1} . \\
\psi_{1}{ }^{1}{ }^{1}: & A^{2}{ }_{1}=0 . \\
\psi_{2}{ }^{2}{ }_{1}{ }^{2}: & A^{1}{ }_{2}=0 . \\
\psi_{1}{ }^{2}{ }_{1}{ }^{1}: & C^{1}{ }_{2}=0 . \\
\psi_{2}{ }^{1}{ }^{2}: & C^{2}{ }_{1}=0 .
\end{array}
$$


The first five equations imply $A^{1}{ }_{1}=C^{1}{ }_{1}=A^{2}{ }_{2}=C^{2}{ }_{2}$. Thus the most general ground state for 4 sites is

$$
\psi_{\alpha \beta}^{\alpha \beta} E+\psi_{\alpha \beta}^{\beta}{ }^{\delta} B^{\alpha}{ }_{\delta} .
$$

As in the proof of Lemma 2.8 the general inductive step can be reduced to the above argument.

We defined the existence of a gap for an infinite volume ground state in Definition 2.11.

Theorem 5.5. Each of the ground states $\omega^{1}$ and $\omega^{2}$ has a gap.

Proof. Given Theorem 5.1 and Eq. (5.15), the proof is identical to that of Theorem 2.12.

\section{Other Valence Bond Ground States}

There exist generalizations of the VBS states of Sects. 2-4 and the dimerized states of Sect. 5 and Hamiltonians for which these states are exact ground states. We present these states and Hamiltonians here. We do not have any general rigorous results, so this section should be viewed primarily as a list of open questions.

The generalized VBS state may be defined for any lattice with coordination number $z$ and spin $s$ at each site provided $s=n z / 2$ for some integer $n$. The generalized VBS state is formed by constructing $n$ valence bonds at each bond in the lattice. We do this by thinking of the spin $s$ at each site as the symmetrization of $2 s=n z$ spin 1/2's. For example, this generalized VBS state for the onedimensional lattice with spin 2 is shown in Fig. 6.1.

A Hamiltonian for which this state is an exact ground state is

$$
H=\sum_{(i, j) \in \mathbb{B}} \sum_{s^{\prime}=2 s-n+1}^{2 s} a_{s^{\prime}} P_{s^{\prime}}\left(\mathbf{S}_{i}+\mathbf{S}_{j}\right),
$$

where the $a_{s^{\prime}}$ are positive constants. The generalized VBS state is a ground state since the restriction of the state to any two adjacent sites contains at least $n$ pairs of spin 1/2's in the singlet state, and so has a total spin of at most $2 s-n$. We expect that the nature of the generalized VBS state depends crucially on the dimension, the coordination number and the multiplicity $n$.

All our rigorous results were for lattices that were bipartite, but we should emphasize that the VBS state and the generalized VBS state can be constructed for lattices which are not bipartite. For example, we may define a VBS state for the triangular lattice with spin 3 at each site. We do not know what properties such a state has, in particular, whether or not the correlation functions decay exponentially.

We can generalize the Majumdar-Ghosh model [31, 32] and some models discovered by Klein [27] as follows. For a lattice with coordination number $z$ and

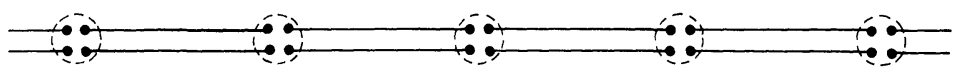

Fig. 6.1. A generalized VBS state 
spin $s$ at each site let

$$
H=\sum_{i} \sum_{s^{\prime}=(z-1) s+1}^{(z+1) s} a_{s^{\prime}} P_{s^{\prime}}\left(\mathbf{S}_{i}+\mathbf{S}_{i_{1}}+\mathbf{S}_{i_{2}}+\ldots+\mathbf{S}_{i_{z}}\right),
$$

where $i_{1}, i_{2}, \ldots, i_{z}$ are the nearest neighbors of the site $i$, and $i$ is summed over the sites in the lattice. Again the $a_{s^{\prime}}$ are positive constants. Now suppose that we have a state in which every site has $2 s$ valence bonds coming out of it and going to some nearest neighbor of that site. (Such states are easily constructed.) Then the restriction of that state to a site $i$ and its $z$ nearest neighbors contains $2 s$ valence bonds and so has a maximum spin of $(z+1) s-2 s=(z-1) s$. Thus, such a state is a ground state of the above Hamiltonian. For the one-dimensional lattice with spin $1 / 2$ at each site we recover the Majumdar-Ghosh Hamiltonian.

If $s$ is an integral multiple of $z / 2$ then the generalized VBS state is a ground state of (6.2). However, the above construction of ground states for (6.2) works even if $s$ is not an integral multiple of $z / 2$. In dimensions greater than one this construction of dimerized ground states yields infinitely many infinite volume ground states. For example, with spin $s=1 / 2$ any covering of the lattice by non-overlapping dimers gives a ground state.

In one dimension, however, we expect that there are only a finite number of ground states for (6.2). For example, some ground states of (6.2) on the onedimensional lattice with spin $3 / 2$ are shown in Fig. 6.2. We refer to the first two states as fully dimerized and the last two as partially dimerized. In the partially dimerized states any pair of adjacent sites always contains at least one valence bond, and so this pair cannot have total spin 3. Thus a Hamiltonian for which the partially dimerized states are ground states, but the fully dimerized states are not, can be constructed by adding to (6.2) the projections onto spin 3 for each pair of adjacent sites. In the fully dimerized state any two sites which are connected by three valence bonds have a total spin of 0 . Thus any three adjacent sites have a total spin of $3 / 2$, and we can form a Hamiltonian for which the fully dimerized states are ground states but the partially dimerized states are not by adding to (6.2) the projection onto spin 1/2 for each group of three adjacent sites. More generally, if we let the sum over $s^{\prime}$ in (6.2) range over all values except $s$, then the

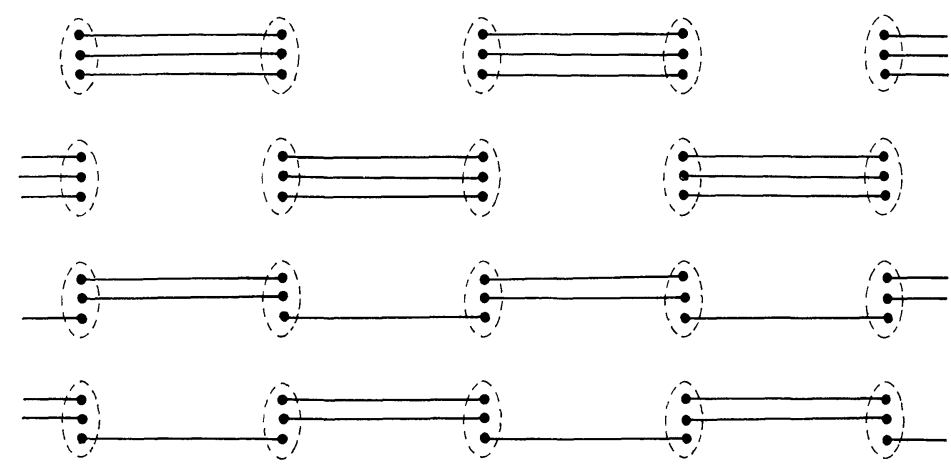

Fig. 6.2. Two fully dimerized ground states and two partially dimerized ground states for the Hamiltonian (6.2) 
resulting Hamiltonian will have the fully dimerized states as ground states, and we expect they are the only ground states.

\section{Appendix A. Néel Order in the Hexagonal Lattice Heisenberg Model}

In Sect. 3 we showed that in the ground state of a particular Hamiltonian on the two-dimensional hexagonal lattice with spin $3 / 2$ at each site the two-point function has exponential decay, and hence there is no Néel order. This raises the possibility that the usual Heisenberg Hamiltonian on the two-dimensional hexagonal lattice does not have Néel order in its ground state. In this appendix we will use the technique of Gaussian domination to rule out this possibility, i.e., to show that the usual Heisenberg Hamiltonian on this lattice does have Néel order provided the spin is at least $3 / 2$.

Gaussian domination was first proven for classical spin systems by Fröhlich et al. [22]. Dyson, Lieb, and Simon then proved the property for quantum antiferromagnets and used it to show the existence of Néel order at low temperature in the usual Heisenberg antiferromagnet on the cubic lattice for spin $\geqq 1$ in dimension $\geqq 3$ and for spin $\geqq 1 / 2$ in dimension $\geqq 4$ [21]. In two dimensions there is no Néel order at finite temperature, but for antiferromagnets it is a nontrivial question whether or not there is Néel order in the ground state. Jordão Neves and Fernando Perez [26] observed that the techniques of Dyson, Lieb, and Simon could be used to prove the existence of Néel order in the ground state for two dimensions if the spin is large enough. For the two-dimensional cubic lattice they claimed that the method proved Néel order if the spin is at least $3 / 2$. Unfortunately, there is a mistake in their calculations. The correct version of inequality (9) of [26] should have a $3 / 2$ in place of the 2 inside the square root in the denominator in the left side of this inequality. One then finds that there is Néel order for the two-dimensional square lattice if the spin is at least 1.

Dyson, Lieb, and Simon pointed out in [21] that their proof of Gaussian domination applies to the two-dimensional hexagonal lattice. Thus it is clear that one can show the ground state for this lattice has Néel order if the spin is large enough. Following [21] and [26], it is a routine calculation to show that $s=3 / 2$ is indeed large enough, so we will only present a few highlights of the calculation.

The only significant difference between the present case and those in [21] and [26] is that we must consider the lattice Laplacian on the hexagonal lattice rather than the usual cubic lattice. This hexagonal lattice Laplacian is defined by

$$
(-\Delta \psi)(x)=3 \psi(x)-\sum_{y:|x-y|=1} \psi(y),
$$

where $y$ is summed over the three nearest neighbors of $x$. To find the eigenvalues and eigenvectors of this operator recall that the two-dimensional hexagonal lattice is bipartite, i.e., it can be written as the disjoint union of two sublattices with any two nearest neighbors belonging to different sublattices. We refer to the sublattices as "even" and "odd."

Let $\delta_{i}, i=1,2,3$ be unit vectors such that for every even site $x$, the nearest neighbors of $x$ are given by $x+\delta_{i}$. The nearest neighbors of an odd site $y$ are then given by $y-\delta_{i}$. For a finite lattice $\Lambda$ with periodic boundary conditions the 
eigenvalues of $-\Delta$ are then

$$
E_{k}^{ \pm}=3 \pm|\varepsilon(k)| \quad \text { with } \quad \varepsilon(k)=\sum_{i=1}^{3} \exp \left(i k \cdot \delta_{i}\right),
$$

where $k$ ranges over the Brillouin zone for one of the two sublattices. The corresponding eigenvectors are

$$
h^{ \pm}{ }_{k}(x)=(\mp)^{x} \exp \left[i k \cdot x+i(-)^{x} \varphi(k) / 2\right]|\Lambda|^{-1 / 2},
$$

where $(-)^{x}$ equals +1 for even $x$ and -1 for odd $x .(+)^{x}$ is always $+1 . \varphi(k)$ is defined by

$$
\varepsilon(k)=|\varepsilon(k)| \exp (i \varphi(k)) .
$$

$|\Lambda|$ denotes the number of sites in the lattice. Note that the number of allowed $k$ 's is $|\Lambda| / 2$, so that we have the correct number of eigenvalues and eigenvectors.

Following [21] we note that there is a unitary transformation (rotation by $\pi$ about the 2-axis at each odd site) which sends $S^{1} \rightarrow(-) S^{1}{ }_{x}, S^{2}{ }_{x} \rightarrow S^{2}{ }_{x}$, $S^{3}{ }_{x} \rightarrow(-)^{x} S^{3}{ }_{x}$. The argument of [21], in particular Lemma 6.1, then proves the Gaussian domination inequality

$$
\left(\sum_{x}(-)^{x} S_{x}^{3} \overline{(-\Delta h)(x)}, \sum_{y}(-)^{y} S_{y}^{3}(-\Delta h)(y)\right) \leqq \frac{1}{\beta} \sum_{x} \overline{h(x)}(-\Delta h)(x),
$$

where $($,$) is the Duhamel two-point function, the bar denotes complex conju-$ gation, and $h(x)$ is any function on the lattice.

Define

$$
S^{ \pm}{ }_{k}=\sum_{x} h_{k}^{ \pm}(x) S^{3}{ }_{x}
$$

We caution the reader that the $S^{ \pm}{ }_{k}$ should not be confused with the raising and lowering operators. $S^{ \pm}{ }_{k}$ is a linear combination only of $S^{3}{ }_{x}$. We now insert one of the eigenvectors $h^{ \pm}{ }_{k}$ for $h$ in (A.4). Since $(-)^{x} h^{ \pm}{ }_{k}(x)=h^{\mp}{ }_{k}(x)$, this yields

$$
\left(\overline{S^{ \pm}}, S^{ \pm}{ }_{k}\right)<1 /\left(\beta E^{\mp}{ }_{k}\right) \text {. }
$$

As in [21], Anderson's lower bound on the ground state energy and some calculation gives an upper bound on the expectation of the double commutator

$$
\begin{aligned}
& \left\langle\left[S^{ \pm}{ }_{k},\left[H, \overline{S^{ \pm}}{ }_{k}\right]\right]\right\rangle \leqq C^{ \pm}{ }_{k}, \\
& C^{ \pm}{ }_{k}=2 S(S+1 / 3) E^{ \pm}{ }_{k} / 3 \text {. }
\end{aligned}
$$

Following [26], the Gaussian domination inequality (A.6), the Falk-Bruch inequality, the usual sum rule and $\left\langle S^{3}{ }_{x} S^{3}{ }_{x}\right\rangle=\left\langle\mathbf{S}_{x} \cdot \mathbf{S}_{x}\right\rangle / 3=S(S+1) / 3$ imply there is Néel order in the ground state if

$$
2 S(S+1) / 3>\lim _{\Lambda \rightarrow \infty} \frac{1}{|\Lambda|} \sum_{s=-,+} \sum_{k}\left[2 S(S+1 / 3) E_{k}^{-s} /\left(3 E_{k}^{s}\right)\right]^{1 / 2} .
$$

Define

$$
\begin{aligned}
I & =\lim _{\Lambda \rightarrow \infty} \frac{1}{|\Lambda|} \sum_{s=-,+} \sum_{k}\left[E_{k}^{-s} / E_{k}^{s}\right]^{1 / 2} \\
& =\frac{1}{2|B|} \int_{B} d^{2} k\left\{\left[E^{-}{ }_{k} / E^{+}{ }_{k}\right]^{1 / 2}+\left[E^{+}{ }_{k} / E^{-}{ }_{k}\right]^{1 / 2}\right\},
\end{aligned}
$$


where $B$ is the Brillouin zone for one of the sublattices and $|B|$ is its area. Then (A.8) becomes

$$
(S+1)[2 S /(3 S+1)]^{1 / 2}>I .
$$

For $S=3 / 2$ the left side is $1.846 \ldots$, and numerical evaluation of the integral yields $I=1.52 \pm 0.01$. Thus there is Néel order for spin $3 / 2$ and higher.

Our calculation has been for the two-dimensional hexagonal lattice, but there is an analogous calculation for any bipartite lattice for which Gaussian domination holds. The integral $I$ depends on the lattice type and the dimension, but $I$ is always at least 1 because of the trivial inequality $x+1 / x \geqq 2$. As the dimension $d$ increases, $I$ typically decreases to 1 . For example, for the cubic lattice $I=1+O(1 / d)[21]$. The coordination number $z$ enters the above calculation only in the bound on the double commutator (A.7). Condition (A.9) then becomes

$$
(S+1)[2 S z / 3(S z+1)]^{1 / 2}>I \text {. }
$$

Acknowledgement. It is a pleasure to thank Mariko Tasaki for drawing the figures.

\section{References}

1. Affleck, I., Kennedy, T., Lieb, E.H., Tasaki, H.: Rigorous results on valence-bond ground states in antiferromagnets. Phys. Rev. Lett. 59, 799-802 (1987)

2. Affleck, I.: Large- $n$ limit of $S U(n)$ quantum spin chains. Phys. Rev. Lett. 54, 966-969 (1985)

3. Affleck, I.: Exact critical exponents for quantum spin chains, nonlinear $\sigma$-models at $\theta=\pi$ and the quantum Hall effect. Nucl. Phys. B 265, 409-447 (1986)

4. Affleck, I., Haldane, F.D.M.: Critical theory of quantum spin chains. To appear in Phys. Rev. B.

5. Affleck, I., Lieb, E.H.: A proof of part of Haldane's conjecture on spin chains. Lett. Math. Phys. 12, 57-69 (1986)

6. Aizenman, M., Lieb, E.H.: The third law of thermodynamics and the degeneracy of the ground state for lattice systems. J. Stat. Phys. 24, 279-297 (1981)

7. Anderson, P.: The $s=1 / 2$ antiferromagnetic ground state: Néel antiferromagnet or quantum liquid. Mat. Res. Bull. 8, 153 (1973)

8. Anderson, $\mathrm{P}$.: The resonating valence bond state in $\mathrm{La}_{2} \mathrm{CuO}_{4}$ and superconductivity. Science 235, 1196-1198 (1987)

9. Babudjian, J.: Exact solution of the one-dimensional isotropic Heisenberg chain with arbitrary spins $S$. Phys. Lett. 90 A, 479-482 (1982)

10. Babudjian, J.: Exact solution of the isotropic Heisenberg chain with arbitrary spins: thermodynamics of the model. Nucl. Phys. B 215, 317-336 (1983)

11. Bethe, H.: Zur Theorie der Metalle. I. Eigenwerte und Eigenfunktionen der linearen Atomkette. Z. Phys. 71, 205-226 (1931)

12. Blote, H.J., Nightingale, M.P.: Gap of the linear spin-1 Heisenberg antiferromagnet: a MonteCarlo calculation. Phys. Rev. B 33, 659-661 (1986)

13. Bonner, J.C.: Private communication

14. Bratteli, O., Robinson, D.W.: Operator algebras and quantum statistical mechanics. II. Berlin, Heidelberg, New York: Springer 1981

15. van den Broek, P.M.: Exact value of the ground state energy of the linear antiferromagnetic Heisenberg chain with nearest and next-nearest neighbour interactions. Phys. Lett. $77 \mathrm{~A}$, 261-262 (1980) 
16. Buyers, W., Morra, R., Armstrong, R., Hogan, M., Gerlack, P., Hirakawa, K.: Experimental evidence for the Haldane gap in a spin-1, nearly isotropic, antiferromagnetic chain. Phys. Rev. Lett. 56, 371-374 (1986)

17. Caspers, W.J.: Exact ground states for a class of linear antiferromagnetic spin systems. Physica 115 A, 275-280 (1982)

18. Caspers, W.J., Magnus, W.: Exact ground states for a class of linear quantum spin systems. Physica 119 A, 291-294 (1983)

19. Caspers, W.J., Magnus, W.: Some exact excited states in a linear antiferromagnetic spin system. Phys. Lett. 88 A, 103-105 (1982)

20. Chang, K.: Calculation of the singlet-singlet gap in spin-1, antiferromagnetic quantum spin chains using valence bond diagrams. Senior thesis, Princeton University (1987)

21. Dyson, F.J., Lieb, E.H., Simon, B.: Phase transitions in quantum spin systems with isotropic and nonisotropic interactions. J. Stat. Phys. 18, 335-383 (1978)

22. Fröhlich, J., Simon, B., Spencer, T.: Infrared bounds, phase transitions, and continuous symmetry breaking. Commun. Math. Phys. 50, 79-95 (1976)

23. Haldane, F.D.M.: Continuum dynamics of the $1-d$ Heisenberg antiferromagnet: identification with the $O(3)$ nonlinear sigma model. Phys. Lett. 93 A, $464-468$ (1983)

24. Haldane, F.D.M.: Nonlinear field theory of large-spin Heisenberg antiferromagnets: semiclassically quantized solutions of the one-dimensional easy-axis Néel state. Phys. Rev. Lett. 50, 1153-1156 (1983)

25. Haldane, F.D.M.: " $\theta$ physics" and quantum spin chains (abstract). J. Appl. Phys. 57, 3359 (1985)

26. Jordão Neves, E., Fernando Perez, J.: Long range order in the ground state of twodimensional antiferromagnets. Phys. Lett. 114 A, 331-333 (1986)

27. Klein, D.J.: Exact ground states for a class of antiferromagnetic Heisenberg models with short-range interactions. J. Phys. A 15, 661-671 (1982)

28. Kulish, P.P., Reshetikhin, N.Yu., Sklyanin, E.K.: Yang-Baxter equation and representation theory. I. Lett. Math. Phys. 5, 393-403 (1981)

29. Kulish, P.P., Sklyanin, E.K.: Quantum spectral transform method: recent developments. In: Integrable quantum field theories. Ehlers, J., Hepp, K., Kippenhahn, R., Weidenmüllen, H.A., Zittartz, J. (eds.). Lecture Notes in Physics, Vol. 151, pp. 61-119. Berlin, Heidelberg, New York: Springer 1982

30. Lieb, E.H., Mattis, D.J.: Ordering energy levels of interacting spin systems. J. Math. Phys. 3, 749-751 (1962)

31. Majumdar, C.K., Ghosh, D.K.: On next nearest-neighbor interaction in linear chain. I, II. J. Math. Phys. 10, 1388-1402 (1969)

32. Majumdar, C.K.: Antiferromagnetic model with known ground state. J. Phys. C 3, 911-915 (1970)

33. Nienhuis, B.: Exact critical point and critical exponents of $O(n)$ models in two dimensions. Phys. Rev. Lett. 49, 1062-1064 (1982)

34. Oitmaa, J., Parkinson, J.B., Bonner, J.C.: Crossover effects in a general spin-1-bilinearbiquadratic exchange Hamiltonian. J. Phys. C 19, L595-L601 (1986)

35. Reed, M., Simon, B.: Methods of modern mathematical physics. I. Functional analysis. New York: Academic Press 1972

36. Renard, J.P., Verdaguer, M., Regnault, L.P., Erkelens, W.C., Rossatmignod, J., Stirling, W.G.: Presumption for a quantum energy gap in the quasi-one-dimensional $s=1$ Heisenberg antiferromagnet $\mathrm{Ni}\left[\mathrm{C}_{2} \mathrm{H}_{8} \mathrm{~N}_{2}\right]_{2} \mathrm{NO}_{2}\left[\mathrm{ClO}_{4}\right]$. To appear in Europhys. Lett.

37. Schulz, H.J., Ziman, T.: Finite-length calculations of $\eta$ and phase diagrams of quantum spin chains. Phys. Rev. B 33, 6545-6548 (1986)

38. Shastry, B.S., Sutherland, B.: Exact ground state of a quantum mechanical antiferromagnet. Physica 108 B, 1069-1070 (1981)

39. Shastry, B.S., Sutherland, B.: Excitation spectrum of a dimerized next-neighbor antiferromagnetic chain. Phys. Rev. Lett. 47, 964-967 (1981) 
40. Shastry, B.S., Sutherland, B.: Exact solution of a large class of interacting quantum systems exhibiting ground state singularities. J. Stat. Phys. 33, 477-484 (1983)

41. Solyom, J.: Competing bilinear and biquadratic exchange couplings in spin 1 Heisenberg chains. Institute Laue-Langevin preprint

42. Takhtajan, L.: The picture of low-lying excitations in the isotropic Heisenberg chain of arbitrary spins. Phys. Lett. 87 A, 479-482 (1982)

43. Wells, A.F.: Three-dimensional nets and polyhedra. New York: Wiley 1977

44. Wreszinsky, W.F.: Charges and symmetries in quantum theories without locality. Fortschr. Phys. 35, 379-413 (1987)

45. Landau, L., Fernando-Perez, J., Wreszinski, W.F.: Energy gap, clustering, and the Goldstone theorem in statistical mechanics. J. Stat. Phys. 26, 755-766 (1981)

46. Wreszinski, W.F.: Goldstone's theorem for quantum spin systems of finite range. J. Math. Phys. 17, 109-111 (1976)

47. Arovas, D.P., Auerbach, A., Haldane, F.D.M.: Extended Heisenberg models of antiferromagnetism: Analogies to the quantum Hall effect. University of Chicago preprint

Communicated by A. Jaffe

Received August 20, 1987

Note added in proof. After the completion of this work we received a preprint [47] by D.P. Arovas, A. Auerbach and F.D.M. Haldane which contains a new representation of the VBS state. Using this representation we have proved some of the conjectures of the present paper including the uniqueness of the infinite volume ground state (and hence the absence of Néel order) for the hexagonal lattice model of Sect. 3. These results are now being prepared for publication. 